Florida International University FIU Digital Commons

$3-5-2010$

\title{
Realization of Differentiated Quality of Service for Wideband Code Division Multiple Access Core Network
}

Yechang Fang

Florida International University, yfang003@fiu.edu

DOI: $10.25148 /$ etd.FI10080417

Follow this and additional works at: https://digitalcommons.fiu.edu/etd

\section{Recommended Citation}

Fang, Yechang, "Realization of Differentiated Quality of Service for Wideband Code Division Multiple Access Core Network" (2010). FIU Electronic Theses and Dissertations. 244.

https://digitalcommons.fiu.edu/etd/244 


\section{FLORIDA INTERNATIONAL UNIVERSITY}

Miami, Florida

\section{REALIZATION OF DIFFERENTIATED QUALITY OF SERVICE FOR WIDEBAND CODE DIVISION MULTIPLE ACCESS CORE NETWORK}

A dissertation submitted in partial fulfillment of the

requirements for the degree of DOCTOR OF PHILOSOPHY in ELECTRICAL ENGINEERING

by

Yechang Fang 
To: Dean Amir Mirmiran

College of Engineering and Computing

This dissertation, written by Yechang Fang, and entitled Realization of Differentiated Quality of Service for Wideband Code Division Multiple Access Core Network, having been approved in respect to style and intellectual content, is referred to you for judgment.

We have read this dissertation and recommend that it be approved.

Jean H. Andrian

Deng Pan

Yimin Zhu

Kang K. Yen, Major Professor

Date of Defense: March 5, 2010

This dissertation of Yechang Fang is approved.

Dean Amir Mirmiran College of Engineering and Computing

Interim Dean Kevin O'Shea University Graduate School

Florida International University, 2010 
(C) Copyright 2010 by Yechang Fang

All rights reserved 


\section{DEDICATION}

I dedicate this thesis to my wife, parents and parents-in-law. Without their patience, understanding, support, and most of all love, the completion of this work would not have been possible. 


\section{ACKNOWLEDGMENTS}

I wish to thank my advisor, Dr. Kang Yen, for his support, patience, and good humor. His gentle but firm direction has been most appreciated. His interest in sense of telecommunication was the impetus for my proposal. From the beginning, he had confidence in my abilities to not only complete a degree, but to complete it with excellence. I extend my gratitude to my committee members Dr. Jean Andrian, Dr. Deng Pan and Dr. Yimin Zhu for their valuable input.

I have found my coursework throughout the curriculum and instruction program at FIU to be stimulating, providing me with the tools with which to explore both past and present ideas and research issues.

The research infrastructure of the System Dynamic Lab and the financial support provided by the Presidential Fellowship and the Dissertation Year Fellowship made of this research endeavor a successful one. 


\begin{abstract}
OF THE DISSERTATION
REALIZATION OF DIFFERENTIATED QUALITY OF SERVICE FOR WIDEBAND

CODE DIVISION MULTIPLE ACCESS CORE NETWORK

by
\end{abstract}

Yechang Fang

Florida International University, 2010

Miami, Florida

Professor Kang K. Yen, Major Professor

The development of $3 \mathrm{G}$ (the 3rd generation telecommunication) value-added services brings higher requirements of Quality of Service (QoS). Wideband Code Division Multiple Access (WCDMA) is one of three 3G standards, and enhancement of QoS for WCDMA Core Network $(\mathrm{CN})$ becomes more and more important for users and carriers. The dissertation focuses on enhancement of QoS for WCDMA CN. The purpose is to realize the DiffServ (Differentiated Services) model of QoS for WCDMA CN.

Based on the parallelism characteristic of Network Processors (NPs), the NP programming model is classified as Pool of Threads (POTs) and Hyper Task Chaining (HTC). In this study, an integrated programming model that combines both of the two models was designed. This model has highly efficient and flexible features, and also solves the problems of sharing conflicts and packet ordering. We used this model as the programming model to realize DiffServ QoS for WCDMA CN.

The realization mechanism of the DiffServ model mainly consists of buffer management, packet scheduling and packet classification algorithms based on NPs. First, 
we proposed an adaptive buffer management algorithm called Packet Adaptive Fair Dropping (PAFD), which takes into consideration of both fairness and throughput, and has smooth service curves. Then, an improved packet scheduling algorithm called Priority-based Weighted Fair Queuing (PWFQ) was introduced to ensure the fairness of packet scheduling and reduce queue time of data packets. At the same time, the delay and jitter are also maintained in a small range. Thirdly, a multi-dimensional packet classification algorithm called Classification Based on Network Processors (CBNPs) was designed. It effectively reduces the memory access and storage space, and provides less time and space complexity.

Lastly, an integrated hardware and software system of the DiffServ model of QoS for WCDMA CN was proposed. It was implemented on the NP IXP2400. According to the corresponding experiment results, the proposed system significantly enhanced QoS for WCDMA CN. It extensively improves consistent response time, display distortion and sound image synchronization, and thus increases network efficiency and saves network resource. 


\section{TABLE OF CONTENTS}

CHAPTER

PAGE

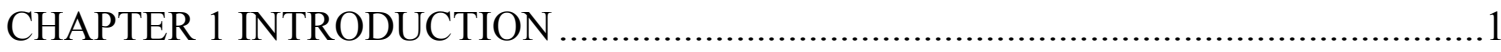

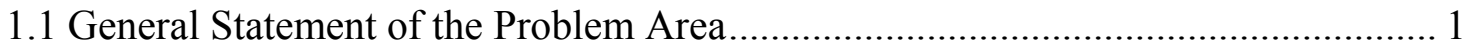

1.2 Significance of the Study ……………............................................................. 3

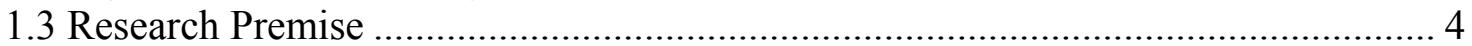

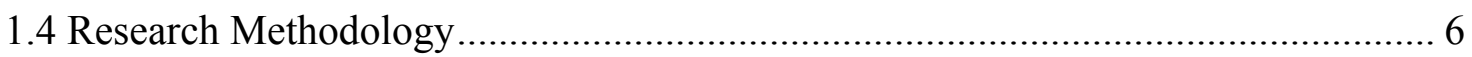

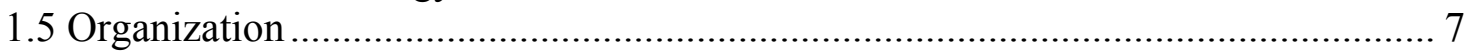

CHAPTER 2 REALIZATION MECHANISM OF DIFFERSEV QOS FOR WCDMA

$\mathrm{CN}$ .9

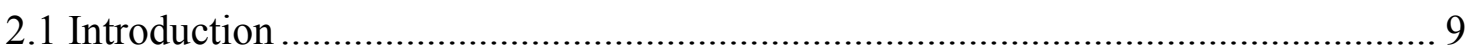

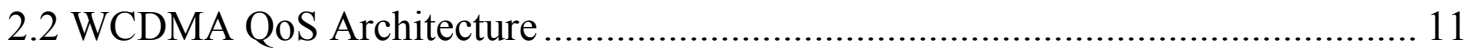

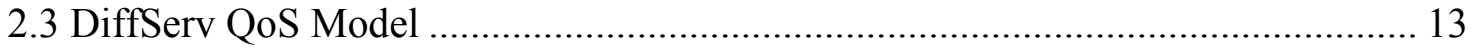

2.3.1 DiffServ Architecture..................................................................... 13

2.3.2 DiffServ Features and Advantages....................................................... 15

2.4 DiffServ QOS Realization Mechanism ................................................................ 17

2.4.1 DiffServ Realization Framework .......................................................... 17

2.4.2 DiffServ Realization Mechanism ..................................................... 18

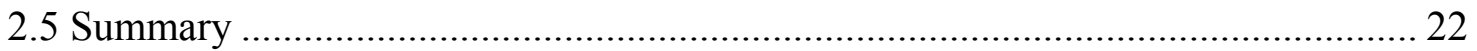

CHAPTER 3 HARDWARE PLATFORM AND PROGRAMMING MODEL OF

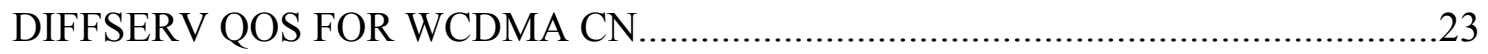

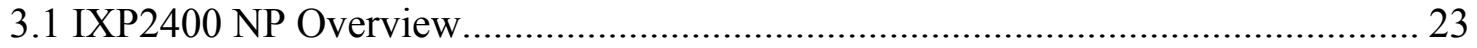

3.2 IXP2400 Functional Modules ……………………........................................ 25

3.3 Advantages of Using IXP2400 to Achieve DiffServ QoS ....................................... 28

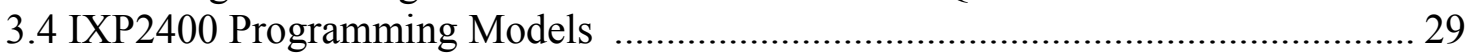

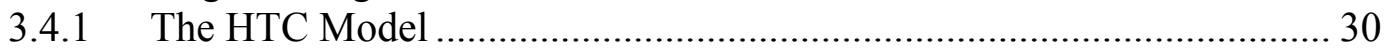

3.4.2 The POTs Model .................................................................................. 32

3.5 Development of the Comprehensive Programming Model …………………........ 33

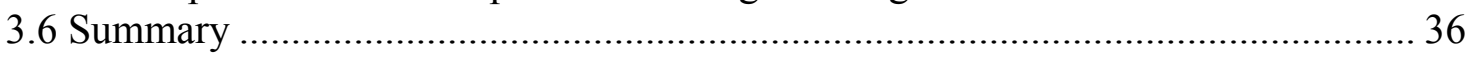

CHAPTER 4 QUEUE MANAGEMENT ALGORITHM DESIGN …...............................37

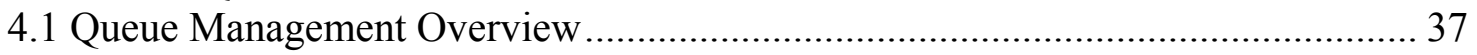

4.2 Existing Buffer Management Algorithms ........................................................... 37

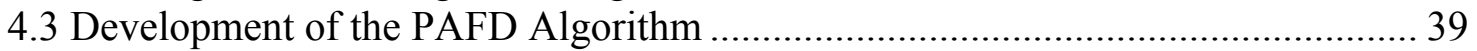

4.3.1 Algorithm Description....................................................................... 40

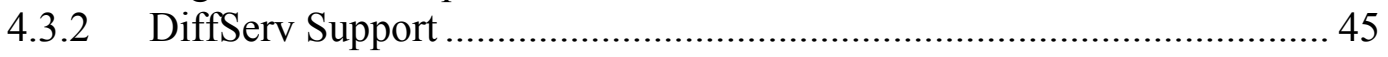

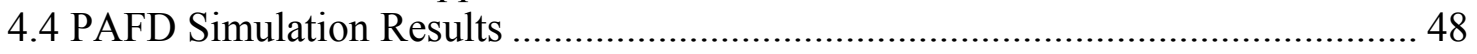

4.4.1 Simulation for Commen Services …………......................................... 48

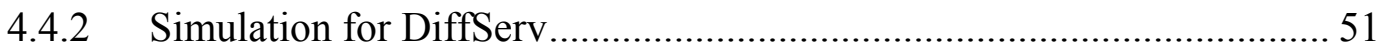

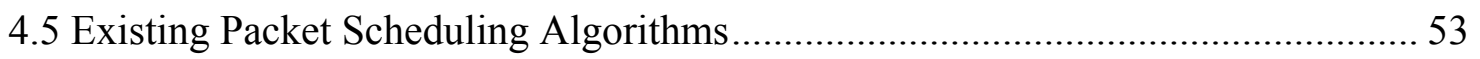

4.6 Development of the PWFQ Algorithm ................................................................ 56 


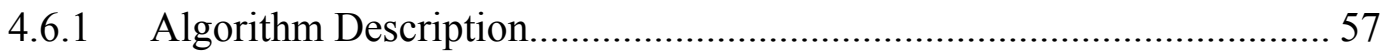

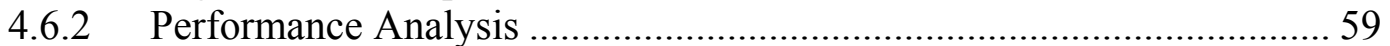

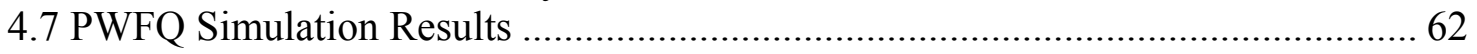

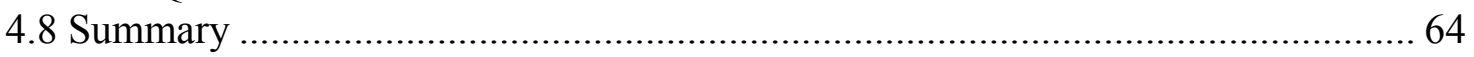

CHAPTER 5 PACKET CLASSIFICATION ALGORITHM DESIGN..............................65

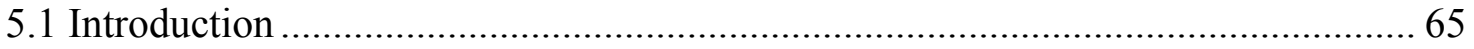

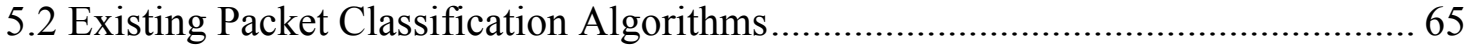

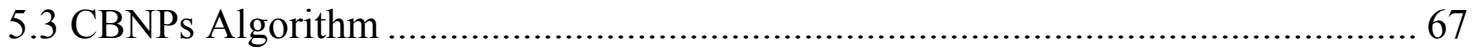

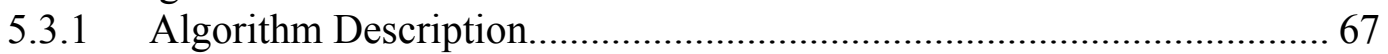

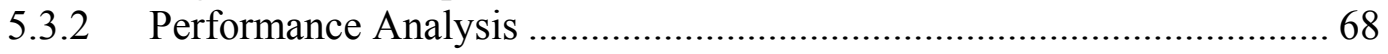

5.3.3 Algorithm Implementation and Optimization ........................................ 70

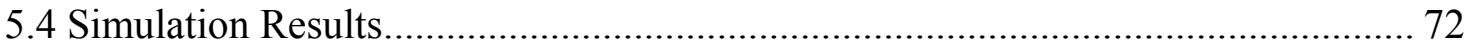

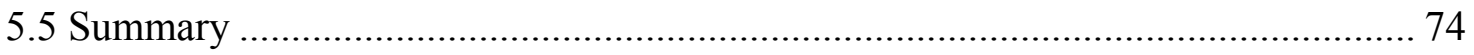

CHAPTER 6 REALIZATION OF DIFFSERV QOS FOR WCDMA CN.........................75

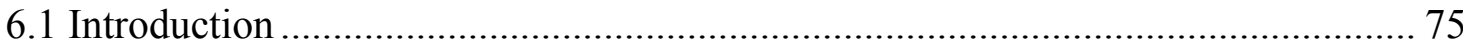

6.2 Hardware Design of WCDMA QoS Based on IXP2400 ..................................... 76

6.3 Software Design of WCDMA QoS Based on IXP2400 ..................................... 78

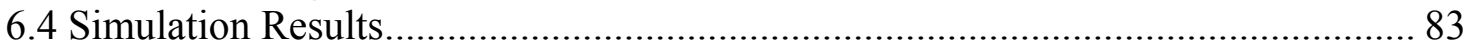

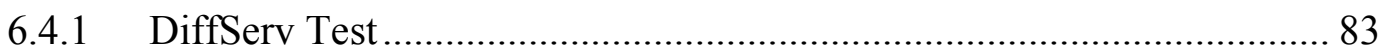

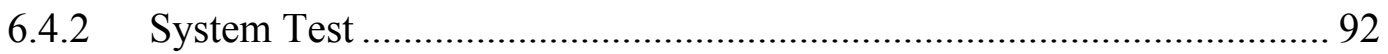

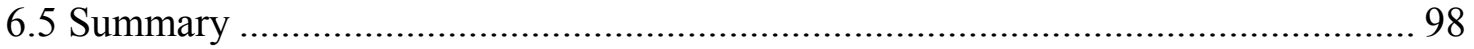

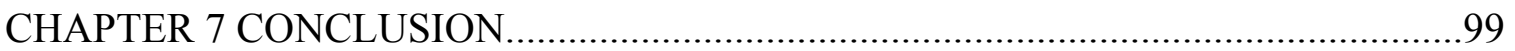

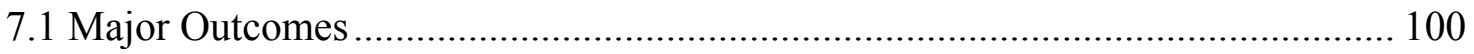

7.2 Prospective Research Endeavors...................................................................... 101

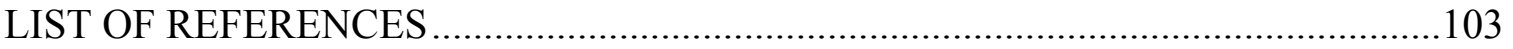

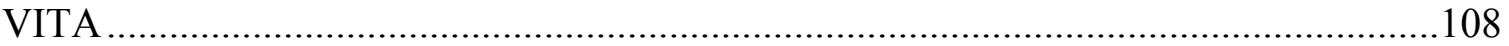




\section{LIST OF TABLES}

TABLE

PAGE

Table 4-1 Fairness Index Comparison of TD, RED and PAFD .....................................51

Table 4-2 Fairness Index Comparison between TD and DS-PAFD .............................. 53

Table 4-3 Comparison of Algorithm Results ........................................................62

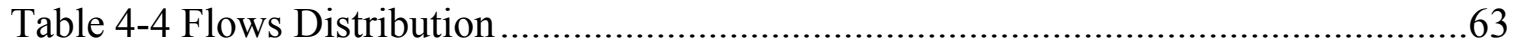

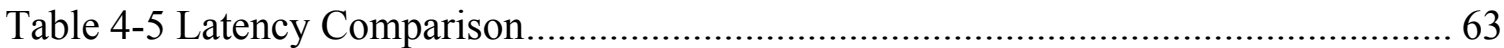

Table 5-1 Measured Results from Real Application..................................................... 74

Table 6-1 Test Equipments and Simulation Modules.................................................. 93

Table 6-2 Startup Delay Comparison Results........................................................... 96

Table 6-3 System Capacity Comparison Results....................................................... 98 


\section{LIST OF FIGURES}

FIGURE

PAGE

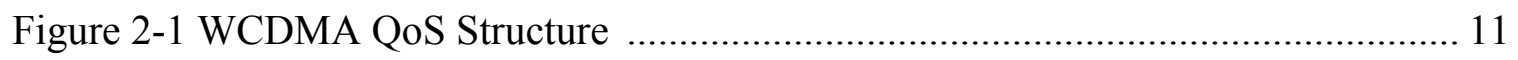

Figure 2-2 Control Information Interactive Process .............................................. 21

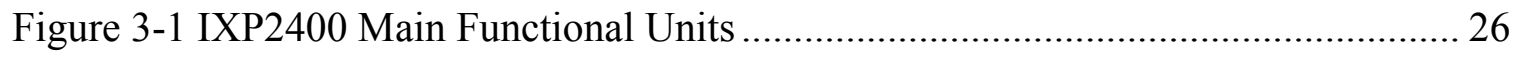

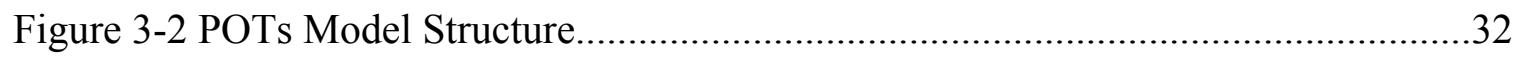

Figure 3-3 Comprehensive Programming Model Structure.......................................... 35

Figure 4-1 An Adaptive Curve of Parameter a ..................................................... 45

Figure 4-2 Values of Parameter $\alpha$ for Different Priority Services ................................46

Figure 4-3 Values of Parameter $\beta$ for Different Priority Services ............................... 47

Figure 4-4 Throughput Comparison between RED and PAFD ................................. 49

Figure 4-5 Comparison of Average Queuing Delay for TD, RED and PAFD ................ 50

Figure 4-6 Throughput Comparison between RED and DS-PAFD............................... 52

Figure 4-7 Comparison of Average Queuing Delay for RED and DS-PAFD ................ 52

Figure 4-8 A Typical Packet Scheduling System ................................................. 54

Figure 6-1 Positions of QoS Switching in a Network............................................... 76

Figure 6-2 Hardware Architecture of DiffServ QoS...................................................77

Figure 6-3 Software Architecture of DiffServ QoS ................................................. 79

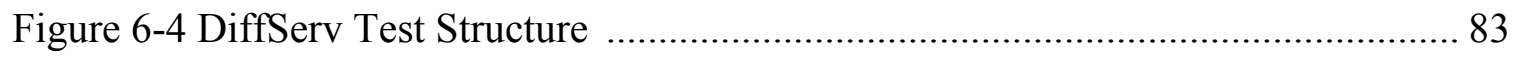

Figure 6-5 (a) K1297-G20 Signaling Analyzer (b) The Interface of K1297 .................. 84

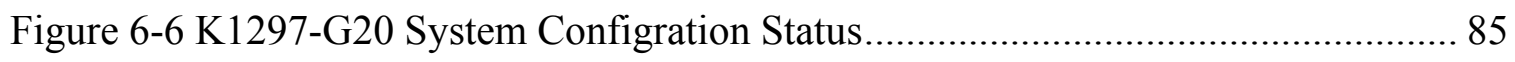

Figure 6-7 (a) Test Type Options (b) Data Flow Setup ..............................................86 


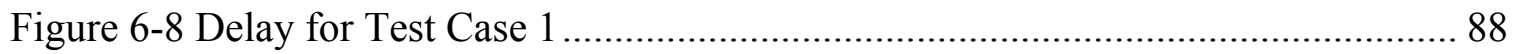

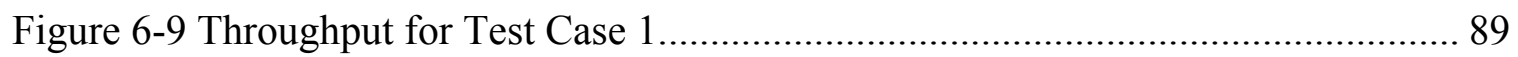

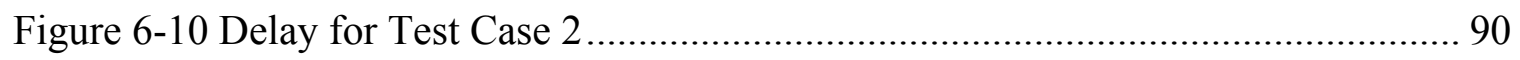

Figure 6-11 Throughput for Test Case 2 .......................................................... 90

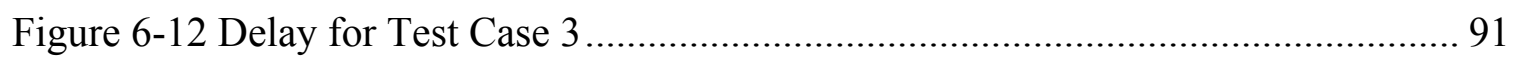

Figure 6-13 Throughput for Test Case 3 ............................................................ 91

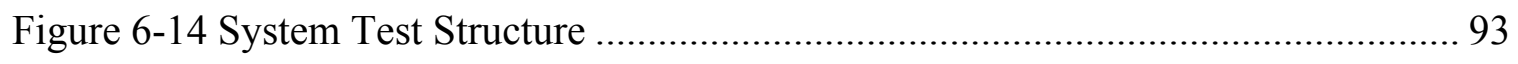

Figure 6-15 Startup Delay Comparison Diagram .................................................. 96

Figure 6-16 System Capacity Comparison Diagram ................................................ 97 


\section{CHAPTER 1}

\section{INTRODUCTION}

\subsection{General Statement of Problem Area}

As the development of 3G, 3G QoS has become the research focus of the network carriers. Wireless communication systems based on switch circuits will eventually evolve into an end-to-end all-IP network, which uses IP networks as the core, and wireless networks as the access approach. It also provides voice, video and multimedia operations with QoS assurance mechanisms [1]. QoS is a kind of measurement for desired performance and priorities of a communication system, which is designed to apply to downlink and uplink connections. As the main model of 3G, Wideband Code Division Multiple Access (WCDMA) has become mature after years of development. It uses a similar structure as Global System for Mobile Communications (GSM) including the Core Network (CN) and the Radio Access Network (RAN) [2-3]. The CN processes voice and data services, and has the capability of switching and routing to external networks. WCDMA CN and all-IP networks are the trend of $3 \mathrm{G}$ development [4-5], and the problem of QoS for WCDMA CN is the extension in mobile communication networks [6].

The QoS assurance provided in IP networks is the development trend of the next generation network (NGN) [7]. IP QoS research aims at providing an effective end-toend QoS control or assurance. A lot of service models and mechanisms, offering different 
levels of QoS assurance for IP networks, have been recommended and standardized, in which the most common ones are IntServ/RSVP (Integrated Services/Resource Reservation Protocol) and DiffServ (Differentiated Services). Compared to the IntServ model, the DiffServ model has better scalability, and can better adapt to the mobile wireless environment, especially suitable for large-scale backhaul networks. Koodli [8] pointed out that the DiffServ model is the most effective method to provide WCDMA CN with QoS guarantee under the current technical condition.

Compared with GSM CN, WCDMA network is committed to providing different types of service with QoS. To achieve the smooth transition of the CN from GSM to WCDMA, the General Packet Radio Service (GPRS) was introduced. GPRS adds packet switching to circuit-switched mode. The Non-Access Stratum (NAS) of WCDMA is horizontally divided into two sub-layers, i.e. mobility management sub-layer and connection management sub-layer. GPRS vertically belongs to two surfaces, i.e. control surface and user surface. Control surface is used for data transmission and processing, while the user surface is for the transmission of user data. The entities which complete the packet-switched service belong to the packet domain. In order to support packetbased operations, two network entities are added. They are Service GPRS Support Node (SGSN) and Gateway GPRS Support Node (GGSN). GGSN uses router architecture that based on NPs. SGSN uses an architecture that includes Asynchronous Transfer Mode (ATM) switches and NP forwarding board. Therefore, the realization of QoS function for WCDMA CN depends on the research and application of NPs. The NP will be the core 
technology promoting the next generation high-performance networks. A lot of current research concentrating on its structure and applications has become a hot topic.

In the WCDMA system, communications between user nodes and internet hosts must be transmitted via the $\mathrm{CN}$. The quality of WCDMA CN services directly impacts user's perceivable feeling. Recently, a lot of QoS realization mechanisms of mobile communication networks have been proposed [9-10], which are constrained in the RAN. Research on QoS for WCDMA CN is still limited. Furthermore, designs of fixed network QoS mechanisms are valuable [11-12], but they do not meet service structures and QoS requirements of WCDMA CN. For various types of QoS operation requirements of WCDMA CN, it is necessary to study the corresponding system models and realization mechanisms.

\subsection{Significance of the Study}

Compared with the previous mobile communication technology, one of the advantages of $3 \mathrm{G}$ is that it gives the carriers more freedom in managing QoS. So that QoS becomes a network management mechanism with strict quantitative definition from a vague concept. From service carriers' perspective, carriers can improve the competitiveness by providing high value-added services in a network, in which an effective control and monitoring of QoS can be carried out. They can also develop different operating strategies for different user services, so the resource utilization can be improved. Since the end-users experience the end-to-end QoS, the carriers are most concerned about how to ensure end-to-end QoS in WCDMA networks. 
To provide differenciated QoS will definitely become the trend of future networks, based on the facts below:

- The diversity of $3 \mathrm{G}$ shifts the attention from price to value.

- The emergence of multimedia services makes people concern more about business experience.

- 3G service diversity makes business model and resource allocation model more complex.

- The diversity in services of 3G makes the design of $3 \mathrm{G}$ networks novel, and more complex.

Enhancement of QoS for WCDMA CN definitely improves consistent response time, display distortion and sound image synchronization to increase network efficiency and save network resource.

\subsection{Research Premise}

The main purpose of this study is to implement the DiffServ model of QoS for WCDMA CN. In WCDMA CN, the main network nodes in Packet Switched (PS) domain come from NPs. Due to this characteristic, this research mainly introduced programming models of NPs and key mechanisms to realize the DiffServ model of QoS for WCDMA $\mathrm{CN}$. Based on the proposed programming model and algorithms, an integrated system is designed to realize the DiffServ model of QoS for WCDMA CN.

1) Programming models of NPs: 
- A comprehensive programming model which combines existing programming models will be designed.

- A solution will be proposed to solve packet ordering and resources exclusive issues.

2) Key realization mechanisms of DiffServ QoS for WCDMA CN:

- The buffer management algorithm: According to the QoS features of WCDMA, a novel algorithm which supports DiffServ will be designed. This algorithm can take consideration of both fairness and throughput, with smooth service curves, and it can also be adjusted in accordance with network traffic.

- The packet scheduling algorithm: By analyzing QoS characteristics of different WCDMA service types, this dissertation will introduce an improved algorithm, which can ensure the fairness of packet scheduling, and reduce queue time of data packets. The algorithm can also keep the delay and wobble within a minimum range, and its performance can be evaluated through a mathematical model of WCDMA packet data.

- The packet classification algorithm: Based on packet compress, rule merger and other algorithms, a new algorithm will be proposed to improve the lookup efficiency, gain desired time and space complexity.

3) Based on a comprehensive programming model and three algorithms proposed in this study, an integrated hardware and software system will be realized on GGSN and 
SGSN equipment based on IXP2400. The effectiveness and performance of the proposed system will be tested by simulations which consist of DiffServ test and system test.

\subsection{Research Methodology}

WCDMA CN is an IP network which provides the $\mathrm{CN}$ bearer services and the associated QoS guarantees. It consists of GGSN and SGSN nodes, and uses tunneling technology to forward packets. In order to use the DiffServ model for QoS control in WCDMA CN, the following issues need to be solved:

1) Appropriate access control schemes must be involved in the design of QoS management functions in the control plane.

2) A variety of WCDMA services must be mapped to Per Hop Behaviors (PHBs) of the DiffServ domain in the design of QoS management functions in the data plane.

Many papers have thoroughly done studies on QoS management in the control plane. However, this study mainly focuses on QoS management in the data plane, which consists of buffer management, packet scheduling and packet classification schemes.

In this dissertation, we used the research method of comparing and empirical study. Firstly, we analyzed and compared the traditional NP programming models, queue management and packet classification algorithms, and pointed out the existing shortcomings. Then, we designed new programming models and algorithms; significance and efficiency of the new algorithms were also tested carefully for the feasibility. Finally, based on the new programming model and algorithms, an integrated hardware and 
software system of DiffServ QoS for WCDMA CN was realized on IXP2400. The research is based on Intel IXA framework and development tool SDK3.0, and the simulation was carried out on K1297-G20 Protocol Tester, Microsoft Visual C++ 6.0 and Microsoft Media Server.

\subsection{Organization}

The study is mainly conducted on QoS for WCDMA CN. Based on summarizing and analyzing the existing research works, a comprehensive programming model for the NP and several new algorithms for QoS control in WCDMA CN were proposed. An integrated system of DiffServ QoS for WCDMA was designed. The main contents of this dissertation are as follows:

Chapter 1 summarized the development of $3 \mathrm{G} \mathrm{CN}$ and the structure of $\mathrm{CN}$ equipment. The DiffServ model of QoS for WCDMA CN was proposed. This chapter also concludes that the NP is an ideal hardware platform to achieve DiffServ QoS for WCDMA CN.

Chapter 2 analyzed the QoS structure of WCDMA CN. Compared with the IntServ model, the advantages of the DiffServ model are outstanding. Realization mechanism of DiffServ QoS was also introduced.

Chapter 3 introduced the research on the NP architecture. In-depth description is carried out on the parallel feature and programming models of NPs. Solutions were proposed to solve sharing conflicts and packet ordering problems that are encountered in 
applications. A comprehensive programming model was proposed as the implementation model of DiffServ QoS for WCDMA CN.

In Chapter 4, Queue management is divided into two aspects, buffer management and packet scheduling. A new buffer management algorithm called PAFD and an improved packet scheduling algorithms called PWFQ were proposed. Theoretical analysis and experiments showed that the algorithms had the following characteristics: (1) to guarantee fair bandwidth allocation, (2) to provide determined upper bound of the delay, (3) to offer less computational complexity, and (4) to take full advantage of the hardware features of network processing.

Chapter 5 proposed a fast packet classification algorithm called CBNPs by using regulation compression and parallel tuple search technology. This algorithm reduces the number of classification rules and classification domain width to accelerate the classification process. It also has high-speed, multi-dimensional and scalable features.

In Chapter 6, based on the programming model and all algorithms proposed in this study, an integrated hardware and software design of DiffServ QoS for WCDMA CN was introduced. The simulation results showed that the proposed system has much better performance than others.

Chapter 7 summarized the research work performed in this dissertation and proposed further research directions. 


\section{CHAPTER 2}

\section{REALIZATION MECHANISM OF DIFFERSEV QOS FOR WCDMA CN}

\subsection{Introduction}

WCDMA networks allow us to provide a variety of communications services in service networks and home environments. The objective of them is to provide a single integrated system. Users can access this system using standard method in a variety of environments. WCDMA defines a number of architectures consisting of logic components with specific functions. An end-to-end WCDMA system includes User Equipment (UE), Universal Mobile Telecommunications System (UMTS) Terrestrial Radio Access Network (UTRAN), the $\mathrm{CN}$ as well as external networks. WCDMA UTRAN is formed by a group of Radio Network Subsystems (RNS), which are access part of UE in the PS domain. It is responsible for achieving the majority of air interface. WCDMA $\mathrm{CN}$ is an IP network which can provide the $\mathrm{CN}$ bearer service and the associated QoS assurance [13]. It consists of GGSN and SGSN nodes, and handles all calls, bearing service support and controls related functions. SGSN implements the mobility management, security management, access control and routing functions. GGSN is responsible for providing interface of GPRS to the outer packet data network. It also provides necessary inter-network security mechanisms, such as firewall. 
The QoS problem in WCDMA networks is more complex than that of general IP networks. Restriction of wireless interface must be taken into account when QoS services are implemented. The complex QoS mechanisms in the fixed network [14] are often unable to adapt to the high error rate at wireless interface. Due to the constraints and robustness of wireless interface, the 3rd Generation Partnership Project (3GPP) [15] defines four types of QoS for WCDMA: Conversation Class, Stream Class, Interaction Class and Background Class. These classifications are based on the delay sensitivity of different operations [16].

WCDMA CN supports services of different QoS levels. Each service is described by a group of QoS parameters. This set of parameters determines the scope of WCDMA bearer services that users can have, and describes the property of users' WCDMA bearer services, which includes the maximum transmission rate, guaranteed transmission rate, transmission order, the largest service data unit, service data unit format, service data unit error ratio, residual error ratio, transmission of the error service data unit, transmission delay, and service process priorities. By treating service types as attributes, WCDMA can first assume the source of a service operation, and then optimizes the transmission of this service.

WCDMA is capable of providing voice, data and multimedia with end-to-end QoS support. Because characteristics of various types of services are significantly different, they require different load-bearing characteristics. 3G network should adopt the appropriate control mechanisms to meet the QoS requirements of different services. For 
the WCDMA network which is based on packet switching, it is particularly important to develop a reliable QoS management mechanism to meet the demand of $3 \mathrm{G}$ services.

\subsection{WCDMA QoS Architecture}

3GPP proposed a hierarchical structure shown in Figure 2-1, which supports endto-end QoS assurance. In order to achieve QoS in WCDMA networks, between the start and the end of service, bearer services whose properties and functions are clearly defined must be established.

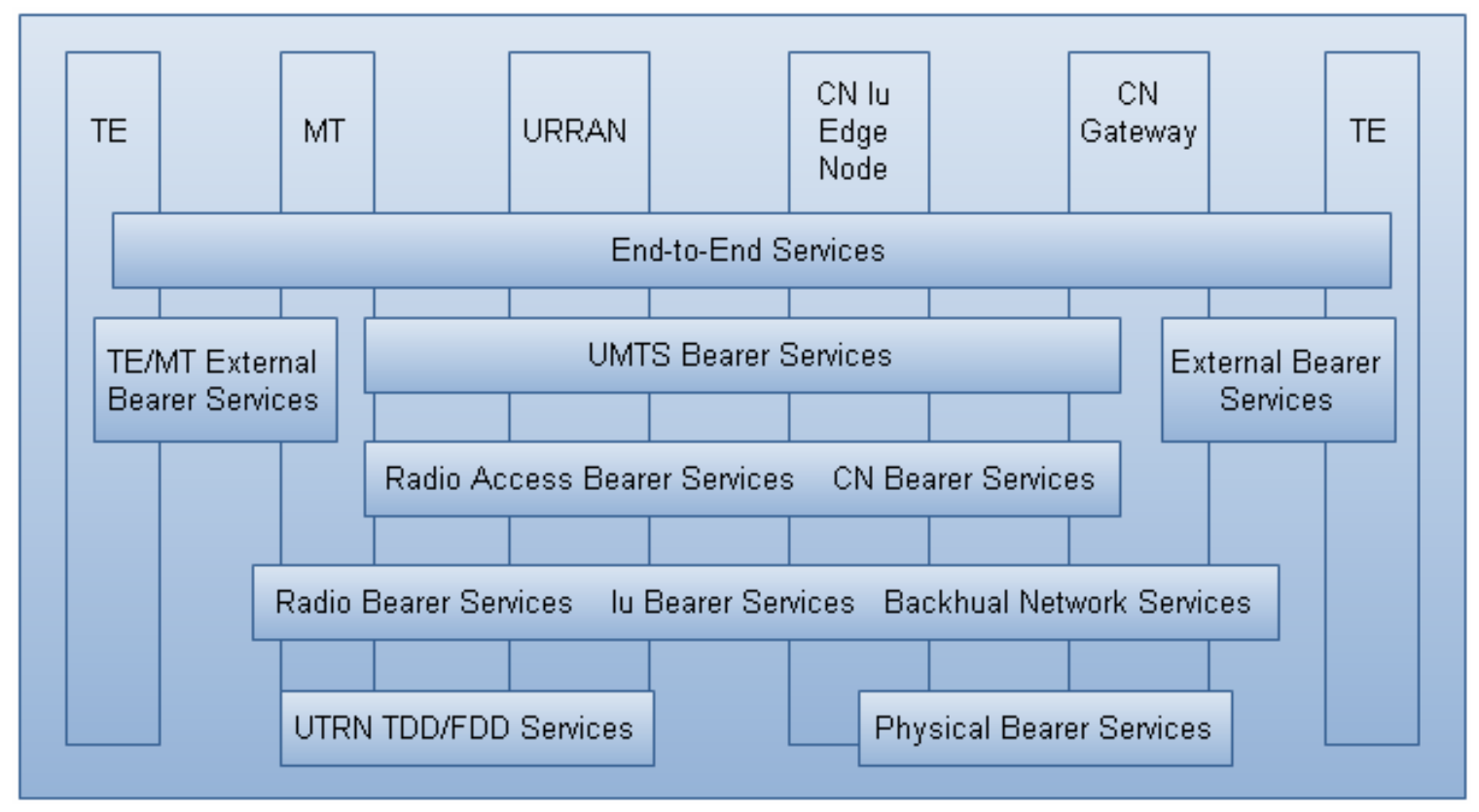

Figure 2-1 WCDMA QoS Structure

In WCDMA networks, the end-to-end service can be decomposed into the terminal equipment/mobile terminal (TE/MT), the local bearer service, WCDMA bearer services and the external bearer service. During the delivery of a service flow from one TE to another TE, the flow must go through different network bearer services. TE/MT 
local bearer service is responsible for the communication between various components of a mobile station (MS). These components can be MTs, and one or multiple of the connected TE. The MT is responsible for physical connection of air interface to WCDMA UTRAN. The TE is connected to the WCDMA network through the MT. The end-to-end services in the application layer uses the underlying network bearer services to provide various services to achieve WCDMA QoS [13]. The external bearer services are responsible for connections between the $\mathrm{CN}$ and external network terminals.

WCDMA bearer services are realized by the Radio Access Bearer (RAB) services and the $\mathrm{CN}$ bearer services. These two services reflect an optimized method to achieve WCDMA bearer services in their respective network topology. Factors to be optimized include the mobility and mobile user properties. The RAB services can provide confidential delivery of signals and user data between the MT and CN Iu edge node, and also satisfy the negotiated QoS of WCDMA bearer services or the default QoS of signals. The $\mathrm{CN}$ bearer services connect the $\mathrm{CN}$ Iu edge node and the $\mathrm{CN}$ gateway to the outside network to effectively control and use the backhaul network to provide the corresponding WCDMA bearer services. WCDMA CN can support various backhaul network bearer services with different QoS requirements.

The $\mathrm{CN}$ bearer services use the common backhaul network services, including the functions of the first and second layers of the network. These functions can be chosen according to the needs of network carriers to meet QoS requirements of the $\mathrm{CN}$ bearer services. WCDMA does not define the backhaul services, so it can take advantage of the 
existing standards. For example, the WCDMA R99 version uses the ATM standard directly, while the bearer services of the IP-based CN use the IETF IP protocol instead.

\subsection{DiffServ QoS Model}

\subsubsection{DiffServ Architecture}

DiffServ uses the classification standard of IntServ. However, it removes the complex admission control algorithms and end-to-end resource reservation protocol. The scalability of DiffServ is improved by classifying and marking the traffic at the edge routers. Moreover, DiffServ does not need complex signaling, so it is particularly suitable for large-scale backhaul networks.

At the edge of the network, DiffServ classifies and forms the single flows entering the network into different flow aggregations. They are then mapped to pre-defined categories. Only a small number of these categories are saved at the core of the network. The router treats the categories of flows differently by using different buffer management and queue scheduling algorithms, and the different treatments brings different service levels. First of all, users and network carriers have to sign a Service Level Agreement (SLA), which defines performance indicators and user flow characterizations. Users pay for data within the scope described in SLA, and the network provides the data with QoS assurance. For the data not specified in the SLA, the network does not provide QoS assurance, or the service level will be reduced. 
When the user traffic enters the network, an edge router measures and classifies the traffic. The user's flows are aggregated into a flow aggregation. The aggregated information is stored in each IP header code point, which is used to tag flow aggregation belonging to IP packets. DiffServ Code Point (DSCP) is located in Types of Service (TOS) at IPv4 header or in Categories of Service (COS) at IPv6 header. Network core equipment conducts scheduling and forwarding according to DSCP, and its external characteristic is called PHB. Overall, the corresponding, forwarding and scheduling behaviors of PHBs are the division of priority. Virtually every PHB corresponds to a priority level. The parameters of each priority specify different requirements on delay, jitter, and packet loss rate. The essence of PHB is the method of resources allocation of each router in DiffServ area for specific flow aggregation. The current standardized PHB includes the default type Best Effort (BE), Class Selector (CS), Expedited Forwarding (EF) [17], and Assured Forwarding (AF) [18]. AF consists of four PHBs. Each Internet Service Provider (ISP) can define different TOS according to their actual needs, such as reward service through $\mathrm{EF}$ and Olympic service through AF.

The DiffServ model uses a hierarchical structure in different ISP networks for coordination. A single ISP network constitutes a DiffServ (DS) domain, and multiple DS domains constitute one DS zone. In a DS domain, the behaviors of DiffServ scheduling forwarding are consistent. DS domains in the same DS zone have to be connected through the edge routers. By using Traffic Condition Agreement (TCA), DS domains provide the cross-DS domain services to ensure consistency in the provision of services to users. 


\subsubsection{DiffServ Features and Advantages}

By studying the DiffServ model, we can summarize the main features of the DiffServ model:

1) There is a sharp distinction between functions of edge routers, core routers and hosts. In the DiffServ model, the routers are further divided into edge routers and core routers. Edge routers keep the per-flow state, SLA information, packet classification, and marking. Core routers do not hold per-flow information, but only keeps a small number of classified information. The core routers also provide different types of packet with different priority treatments.

2) There is a clear contractual relationship between hosts and networks. SLA describes the services that user can expect and the user flow specifications. Difference between SLA and FLOWSPEC which is a list of parameters in IntServ is that SLA is a long-term, static service specification, and the later expresses the exact description for the flow specification of a specific QoS request.

3) Core routers provide different levels of services. In the DiffServ model, the core routers in the backhaul network have only a small number of queues, and flows are converged to a coarse-grained flow aggregation, so that the network can maintain high statistical multiplexing features.

4) The DiffServ model offers a variety of levels of services. According to different bandwidth and delay requirements, DiffServ can provide multiple levels of 
services through different PHBs. From an architectural point of view, DiffServ has the advantages of both IntServ and the traditional Best Effort model. This model maintains the good statistical multiplexing characteristics of the Best Effort model, and has good scalability.

DiffServ is an ideal QoS implementation mechanism in WCDMA CN under the current technology, because

1) The DiffServ architecture and WCDMA CN structures have a certain similarity, therefore the renovation cost is relatively low. SGSN in WCDMA CN is equivalent to the entrance router in DiffServ, so mapping the WCDMA QoS to DiffServ DSCP to achieve the corresponding PHB is all the works. GGSN is similar to the edge router which can improve the flow control function, achieve the inter-regional differential services, and ensure the consistency of QoS.

2) DiffServ does not need complex control signaling, and its good scalability makes it suitable for wireless communications, especially as the QoS control mechanisms in large-scale backhaul networks such as WCDMA CN.

3) Due to the high error rate of wireless interface and heterogeneous network compatibility, WCDMA does not define precise QoS requirements for different TOS. Thereby, this property perfectly matches the characteristic of DiffServ, which does not perform fine granularity QoS control based on the flow aggregation. 


\subsection{DiffServ QOS Realization Mechanism}

\subsubsection{DiffServ Realization Framework}

Different from the IntServ model, the core switch in the DiffServ model forwards and schedules the data packets based on a limited number of COS, rather than the specific circumstances of a session streaming. This requires an important QoS mapping process at the terminal. The physical framework of QoS mapping is different based on different DiffServ implementations. However, a key mapping link in always there. The data packets are mapped into the appropriate COS based on the Relative Priority Index (RPI). In order to satisfy different applications and reduce the edge exchange process, these mapping processes are implemented at the end systems. QoS mapping is an important technology in the implementation of DiffServ. The network edge router provides the services and QoS assurance based on the service category in the specific data packet header. The client can request an overall consideration of QoS requirements and service charges, and map the packets into different service categories, so that the data packets have the corresponding QoS guarantee in the network. Overall the QoS mapping is the basic scheme for DiffServ.

In the DiffServ model, the services are sorted into $i$ categories based on the parameters of QoS, and COS is a set of related QoS parameters. The DiffServ model is reflected as follows: If data packets request category $i$, the edge router provides services according to the standard specified by the QoS parameter set of category $i$. COS is a carrier that transmits QoS information between the terminal and network. According to 
whether the defined type of services has global consistency, DiffServ implementation is divided into two types: absolute implementation and relative implementation. Absolute implementation can provide a more secure end-to-end QoS, but its structure is more complex, and the flexibility and scalability are poor. When the sender conducts adaptive coding and the network edge routers are more intelligent, relative implementation is more flexible and efficient than absolute implementation. In order to separate the encoding details and the network self-adaptive function, we need to define an appropriate evaluation criterion by which each packet is marked with one RPI to correctly represent the importance of the packet in the flow and integrate a variety of factors. An important part to achieve DiffServ QoS is to determine the corresponding DiffServ COS based on the RPI of each data packet, which is so called QoS mapping.

\subsubsection{DiffServ Realization Mechanism}

The DiffServ architecture provides theoretical principles and framework basis for implementation of QoS guarantee mechanism in the IP backhaul network. The specific usage strategies and the corresponding realization of the mechanism are decided by different manufactures. QoS realization mechanism in the DiffServ model is the key to provide QoS guarantee for users. It mainly consists of buffer management, packet scheduling and packet classification mechanism [19-20].

1) Buffer management mechanism is designed to solve the packet-discard problem, when network congestion happens. If the network congestion occurs at the output port, the router buffers must discard some packets to improve the output flow of 
the buffer queue. How to reasonably drop some packets is mainly determined by the buffer management algorithm.

2) Packet scheduling mechanism is the queuing and regulation methods used on data flows to prevent congestion at the network output ports. This mechanism determines how a router selects the next packet from one or more buffer areas and forwards it. Main performance indicators for an efficient packet scheduling algorithm include fairness, delay characteristics, isolation capacity of malicious traffic flow, link bandwidth utilization, and complexity.

3) Packet classification mechanism is mainly used in classifiers and regulators in the DiffServ model. This mechanism ensures that input data flows accord with the Traffic Conditioning Agreement (TCA) and classifies the flows to a certain behavior aggregate, and then tags them as packets correspondingly. The classifier follows the specific rules in TCA to assign packets to a category according to the fields in header such as the DSCP value or the five-factor group of MF, and then hands this category over to the appropriate regulator module for further processing.

In the DiffServ model, EF PHB provides low packet loss rate, delay, and jitter to guarantee bandwidth services. AF PHB is responsible for providing a flow range in the DiffServ domain. The packets within the flow range can receive ensured QoS, and those beyond the range cannot receive guaranteed QoS. AF PHB provides four independent forwarding AF categories, and each category has three different packet loss priorities. $\mathrm{BE}$ is suitable for the traditional Internet service, but does not guarantee QoS. 
According to 3GPP requirements, UE makes the request of QoS by mapping QoS requirements in the end application layer to the Packet Data Protocol (PDP) parameters via an application layer protocol such as the Session Description Protocol (SDP). The management function of WCDMA QoS decides whether to accept the request based on the resource utilization in the UTRAN, SGSN and GGSN. Once the request is allowed, the DiffServ edge node defines the classifier settings and maps different QoS COS to different PHBs based on the DiffServ framework in IP networks. Downlink WCDMA QoS is controlled by a remote host to the GGSN. Then GGSN uses the DiffServ edge function to re-classify the data flow by re-encoding DSCP, and assigns the data to different PDP through DSCP. UE is responsible for controlling PDP content in the process, while GGSN is responsible for providing interaction between the PDP content and DiffServ. The control range of PDP flows is limited to access network part of the WCDMA system between UE and GGSN.

Figure 2-2 shows the detailed interactive flow of QoS control information, in which RAP is the remote access point, and RUE means the remote UE. GGSN completes the conversion from PDP to DiffServ in the uplink, while in the downlink the Flow Template (TFT) completes the conversion from DiffServ to the PDP flow through the TFT filters. 


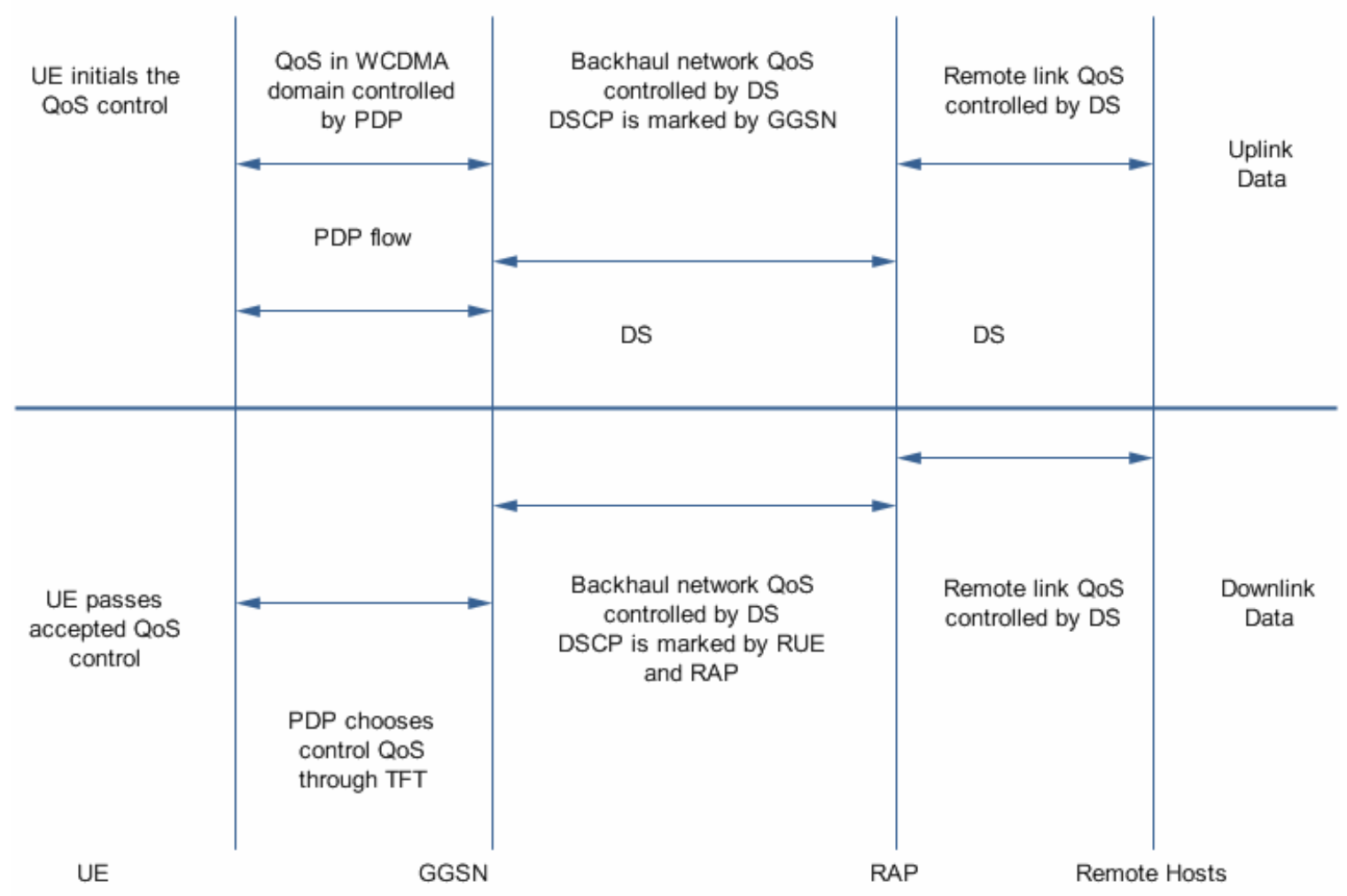

Figure 2-2 Control Information Interactive Process

In the WCDMA COS, the conversation and stream classes which are delaysensitive are mapped to $\mathrm{EF}$ and $\mathrm{AF} 1 \mathrm{PHB}$, respectively, while the interaction and background classes are mapped to AF2 and BE PHB, respectively. Each type of AF PHB can be divided into different sub-types. For example, the interaction services with different QoS requirements can be mapped to AF2 subtypes such as AF21, AF22 and AF23. The mapping from different WCDMA COS to DiffServ PHBs is not unique. It mainly reflects the different QoS requirements of various COS. The QoS control of these service flows is directly determined by queue management algorithms, which include buffer management and packet scheduling algorithms. 


\subsection{Summary}

In this chapter, the WCDMA QoS requirements in 3GPP agreement were analyzed. It is pointed out that the QoS problem in the $\mathrm{CN}$ is an extension of QoS problem in IP networks. By studying on the DiffServ structure, we find that the DiffServ model is more suitable to achieve QoS for WCDMA CN than IntServ. There was also an in-depth analysis of the QoS mapping approach in the DiffServ model and of the realization mechanism in this chapter. The DiffServ model of QoS is mainly

implemented in the data planes of GGSN and SGSN which are the main entities of WCDMA CN. 


\section{CHAPTER 3}

\section{HARDWARE PLATFORM AND PROGRAMMING MODEL OF DIFFSERV QOS FOR WCDMA CN}

\subsection{IXP2400 NP Overview}

As the network scale and interface speed are both increasing, network equipment based on a common Reduction Instruction Set Chip (RISC) technology cannot meet the performance requirements of line-speed processing. On the other hand, new network communication protocols and standards are emerging and changing, and the user's demand is also constantly evolving. As a result, the product cycle of data communication products is greatly shortened. In this background, an NP with both high-speed processing and flexible programming capability provides a flexible solution for design of the next generation communication products [21-23].

An NP is a general purpose chip that dedicates to network communication equipment, which supports an open, diverse, and programmable development environment. In the NP environment, different equipment vendors can use the same chip to create their own network equipment with different functions and features. An NP combines the advantages of RISC and Application Specific Integrated Circuit (ASIC). Just as RISC, an NP is programmable and provides sufficient flexibility to adapt to the fast development of data communication market. It also has high performance as ASIC, but does not take a very long development period. An NP takes into account both 
flexibility of RISC and implementation efficiency of ASIC, and also provides good support for the second-layer to the seventh-layer applications. By downloading different programs, the same hardware platform can support Virtual Local Area Network (VLAN) switches, routers, broadband remote access servers, NAT, firewall, WEB switches and so on. It supports all speeds of Ethernet, ATM, POS and other interfaces, so it is very convenient for upgrading [21].

An NP has a typical multi-core RISC parallel real-time processing architecture. It carries out real-time tasks of packet processing and network bandwidth management. An NP usually consists of a general-purpose processor and multiple parallel or pipelined data packets Processing Engines (PEs) and each internal PE supports multiple threads. Intel's IXP2400 is an NP launched after IXP1200. This NP is based on Intel Exchange Architecture (IXA) [22], and supports $2.5 \mathrm{Gbps}$ applications, with eight $600 \mathrm{MHz}$ microengines and a $600 \mathrm{MHz}$ processing core XScale. One double-data-rate (DDR) SDRAM and two quad-data-rate (QDR) SRAMs can be added to it. IXP2400 also supports standard Media and Switch Fabric (MSF) interface standard SPI-3 or CSIX-L 1. Its Micro-engine processing power is suitable for data packet forwarding and other functions. Eight micro-engines can forward three million packets in Layer 3 per second. The XScale processor can be used to handle complex tasks, such as address learning, establishing and maintaining forward table, and network management. IXP2400 provides high-performance parallel processing capability, with various levels of scalability. Combining the XScale microprocessor with eight separate 32-bit RISC micro packets forwarding engines, as well as hardware multi-threading support, IXP2400 can achieve 
5.4G operations per second to support a wide variety of WAN and LAN applications. By improving the micro-engine architecture, IXP2400 achieves high performance and scalability. These improvements include multi-threading allocation and high-speed cache which gives the software pipeline feature.

\subsection{IXP2400 Functional Modules}

IXP2400 contains eight programmable 32-bit RISC processors which are called micro-engines [23]. The micro-engines are dedicated to handling network traffic. Each micro-engine has eight program counters for eight threads such that we can execute ALU and shift operations in one clock period. Micro-engine instruction set is a data type designed to quickly and efficiently forward packets for networking and communications applications. The set includes bits, bytes and long words.

Micro-engine uses the multi-threading feature, so no extra clock time is needed for thread switching overhead. Each thread has its own thread ID, allowing for the visit results to be returned directly to the requesting thread. When a thread of micro-engines sends a fetch request, this thread switches itself out to let other threads run. It waits for the results from memory and then switches back to run. Thread-switching does not require interrupt mechanism, but is under the control of the micro instructions. This thread switching mechanism can take full advantage of the visit deposit gaps, to improve the micro-engine utilization and throughput. 


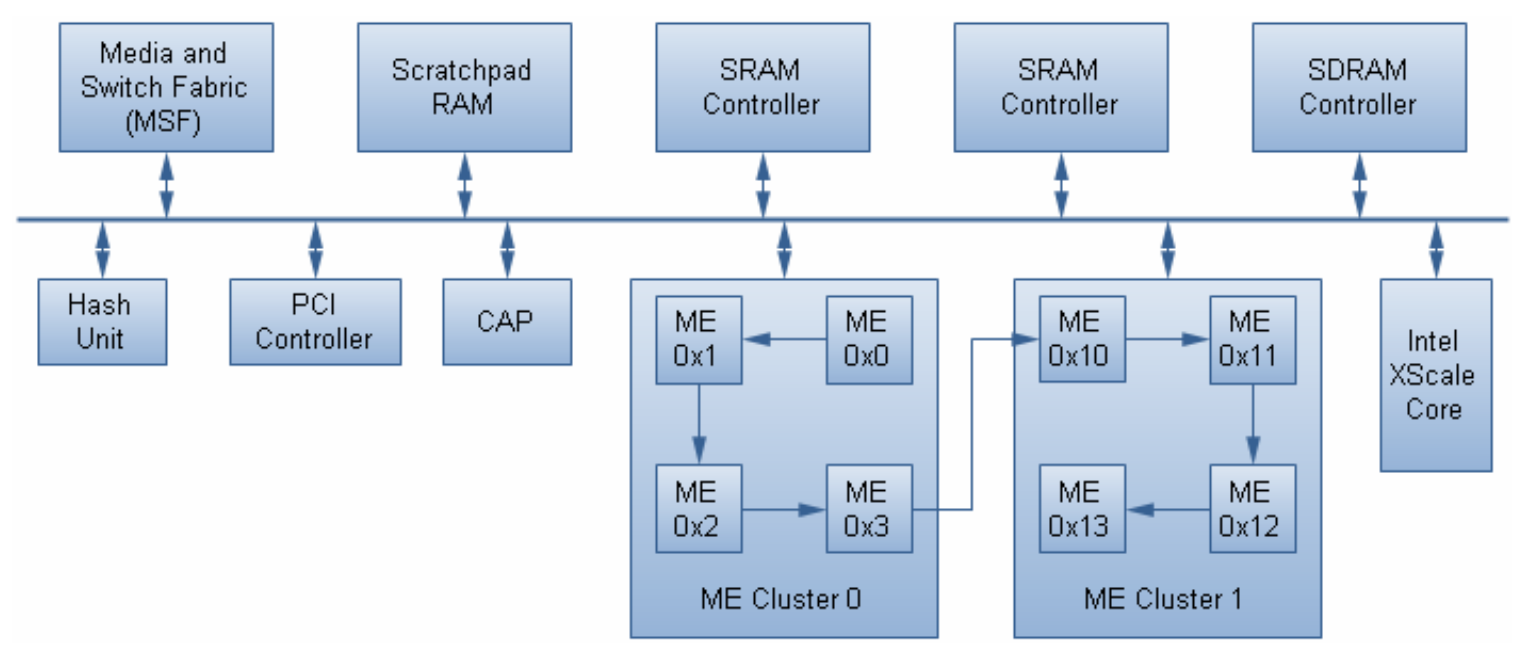

Figure 3-1 IXP2400 Main Functional Units

Figure 3-1 shows a simplified block diagram of IXP2400 main functional units, and the functions of these units are described as below [24].

1) XScale core: It is a complete 32-bit RISC processor with high-performance, low power consumption and compact layout features. It comes with an integrated cache which can be used to achieve management functions, running routing protocols, exception handling and other functions. This unit is applicable to be integrated with other specific operational units in a single chip.

2) Eight micro-engines: These highly efficient RISC engines can be used on any demand for quick detection, data processing, and transmission of packet contents. They are fully programmable 32-bit engines with 5 levels of large pipelined register groups. These micro-engines are multi-threading, and can conduct fast context switching in context-sensitive register windows. 
3) Peripheral Component Interconnect (PCI) bus interface module: This module is a standard interface, which provides possible connection to other PCI devices or other host processors. The PCI interface can reach speed of $133 \mathrm{MHz}$, but this maximum available speed is limited by the PCI bus protocol and bandwidth. There is no internal direct connection between the PCI unit and micro-engines, so PCI is not the primary means of IXP2400 data access.

4) SDRAM, SRAM and Scratchpad RAM modules: The SDRAM module is a shared, intelligent memory interface, which can be accessed by the XScale core, microengines and equipment on the PCI bus. The SRAM module is also a shared intelligent interface, which can be accessed from the XScale and the micro-engine. Scratchpad RAM has small space in IXP2400, and its delay is also very short. It is often used in internal communications, and for shared semaphore and counters. These three storage resources SRAM, SDRAM, and Scratchpad RAM are different in capacity and bandwidth. The benefits of having all three types of storage are that each of these memory operations can be carried out in parallel, and programmers can also select different storage resources based on the requirements of performance. For example, SDRAM memory is suitable for data packets storage and extra large tables, and SRAM memory is for lookup table with shorter delay.

6) MSF interface module: The interface is used to connect IXP2400 to a physical layer device (PHY) and/or to a Switch Fabric. The MSF consists of separate receive and transmit interfaces, which are unidirectional and independent of each other. 


\subsection{Advantages of Using IXP2400 to Achieve DiffServ QoS}

To accelerate the delivery ability of new network services, Intel is providing network equipment vendors and network service providers with the latest secondgeneration Intel IXA NPs. Each NP family is optimized to meet the requirements of target network segments, and is complemented by software tools and development platforms to reduce development cost. We choose IXP2400 as the hardware platform to achieve DIFFSERV QoS for WCDMA CN based on the following reasons:

1) Multi-micro-engine and multi-threading technologies: Each micro-engine is a fully programmable, multi-threaded RISC processor subsystem. It enables highperformance packet processing in the data plane. When the system is running, each micro-engine deals with only one task. During the thread switching, there is no need for protection. This is because each thread has its own register. So the switching speed is very fast.

2) XScale technology: Intel XScale core is designed to control the application processing and for low-layer communications. It manages and updates the data structure shared by routing tables and micro-engines, and establishes and controls the communication media and switching devices. Because the XScale micro-architecture uses the hyper-pipeline technology, it can achieve a relatively high performance. The multi-process and efficient instruction-layer processing pipeline architecture makes the reaction time be reduced to the minimum, and the clock speed is also selected to achieve ultra-low energy consumption. 
3) IXA mobile architecture technology: As described above, Intel IXA mobile architecture provides us with advanced design architecture, so that we can develop products quickly and efficiently.

\subsection{IXP2400 Programming Models}

In general, the data packet processing tasks in practical applications tend to be more complex, so they require multiple micro-engines to work in parallel. This involves how to distribute complex and arduous data tasks among a number of micro-engines, that is, what kind of method can be adopted to map the packet processing tasks to the specific micro-engines. Micro-engine programming model problem is a macro level strategic issue [25], and the study of this problem focuses on how to distribute the data packets among multiple micro-engines in order to obtain the highest processing performance. Micro-engine programming model is universal, not only applicable to eight micro engines in IXP2400, but also to sixteen micro-engines in IXP2800 and the future NPs with more micro-engines.

Packet processing tasks in most applications are complex and diverse, while the micro-engines are fully programmable, so the micro-engine programming models are varied, and are also more flexible choices according to the actual situation. In the following, we introduce two micro-engine programming models. One is known as the super-task chain model such as HTC (Hyper Task Chaining) [26], and the other one is the thread pool model such as POTs (Pool of Threads) [27]. No matter what kind of programming model is chosen, the model must effectively solve two important problems 
in the packet processing. One is maintaining packet ordering, and the other is the thread mutual exclusion problem when multi-threads need to access the same data structure. In mutual exclusion, when a thread is using a data structure for operation, the data structure should be locked to prohibit the use by other threads in order to avoid undermining the integrity of the data. This problem is divided into two situations including inside microengine and among multi-micro-engines. In this chapter, we mainly discuss mutual exclusion encountered in the multi-micro-engine parallel processing problems and the corresponding solutions. Some applications require IXP2400 to maintain the order of packets during the process, and let the data packets leave the IXP2400 according to the arriving order. For example, when dealing with the compressed IPv6 packets, the current data is compressed based on the previous packet, therefore only after the previous packet is processed, the current data packet can be processed. In a word, data packets should be processed in order, which is the so-called packet ordering problem.

\subsubsection{The HTC Model}

HTC is a pipeline style packet processing model $[26,27]$. In this model, the duration of each data packet processing time is relatively fixed, and pre-calculated. Therefore, HTC model is commonly used in the situation that most of the data packets are similar (mainly refers to the case that the packet processing complexity and processing time are basically the same), as well as the situation that data packets belong to the same data flow. In the latter case, we can use the LM, Q-Array, CAM and other hardware structures to quick-fix mutual exclusion problem. In general, using the HTC model for data packet processing can achieve relatively certain processing performance. 
HTC divides a large data packet processing task into several small data packet processing sub-tasks, namely, Network Function $=$ Task $1+$ Task $2+$ Task $3+\ldots+$ Task $n$, Each sub-task can be organized and combined to get another large data packet processing task. There are two combination models as follows:

1) Function Chaining: By using time-division, multiple packet processing tasks are combined in a micro-engine to form a large data packet processing task. To enhance the parallel processing capability, we use multiple micro-engines to run the same packet processing program to process multiple packets. The Function Chaining method is generally suitable for the situation in which multiple processing and substantial changes need to be made for data packets

2) Context Chaining: This model allocates multiple sub-tasks to multiple microengines by using space-division, and then these micro-engines are combined together to form one large data packet processing task. Context Chaining is only used on implementation of single processing and minor modifications for packets.

In general, a series of data packet processing tasks get involved between receiving data packets and adding them into a queue. We can use Function Chaining to link a series of data packet processing tasks together and process them on one micro-engine. For a single micro-engine, packet processing tasks are very heavy, thus the execution speed of Function Chaining is slower than that of Context Chaining. However, we can use multiple micro-engines to work in parallel to improve overall system processing speed. In Context Chaining, one micro-engine is responsible for one packet processing sub-task, 
and unlike the Function Chaining in which one micro-engine is responsible for multiple data packet processing sub-tasks. Therefore, Context Chaining is much simpler than the Function Chaining. This is because that the mutual exclusion problem among multiple micro-engines is not considered.

\subsubsection{The POTs Model}

POTs is also known as "run-to-completion" model [27]. In this model, one program or thread executes most of the data packet processing tasks, such as a series of data packet processing tasks between receiving data packets and executing enqueue operation. The POTs structure is similar to that of the programming model of generalpurpose processors, and has more understandability. The schematic model of POTs is shown in Figure 3-2, in which SPI is Serial Peripheral Interface, and NAT means Network Address Translation.

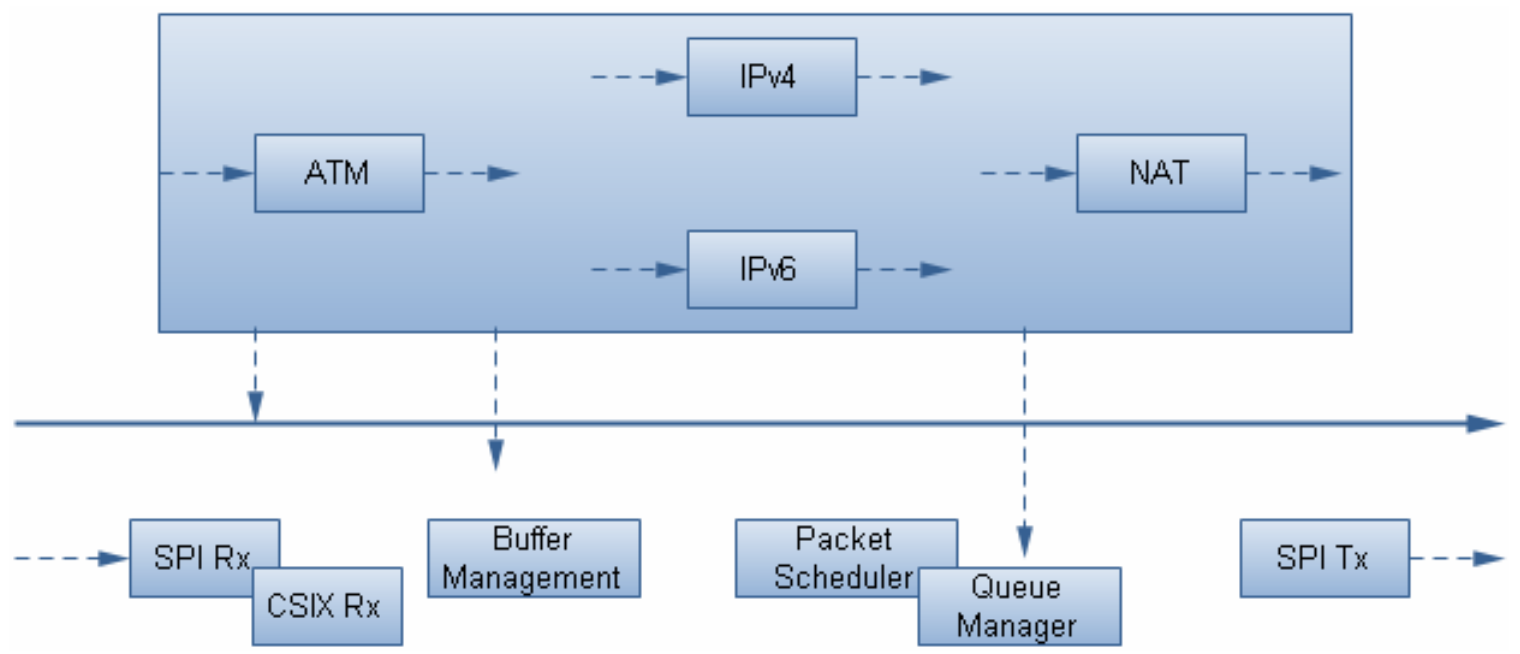

Figure 3-2 POTs Model Structure 
Figure 3-2 shows that the POTs model is based on basic modules which are closely relevant to the underlying hardware architecture. These basic modules including Rx, Buffer Management, Queue Manager and Tx module, are equivalent to the hardware driver modules in the operating system of general-purpose processors. In POTs, DRAM is used for storing data packets, statistical database, forwarding information table and other large data structures. While SRAM memory device is used to store the information associated with data structures such as the packet descriptor, packet status information and Longest Prefix Matching (LPM) lookup table. When the MSF receives a data packet, the packet is cached in the Receive Buffer (RBuf), and then Rx module reads the packet from the RBuf for receiving and reorganizing processing. After that, POTs are used for classification, packet forwarding and other data packet front-end processing tasks. Next, Queue Manager executes enqueue operation, and the packet joins the sending queue. Then the dequeue operation is executed and the packet is removed from the queue. Finally, POTs are used again to complete the back-end data packet processing tasks.

\subsection{Development of the Comprehensive Programming Model}

In the above sections, we analyzed two micro-engine programming models HTC and POTs. Both models have their own advantages and disadvantages. In general, POTs is more flexible and simpler than the HTC, and its scalability is also better. For example, when the system hardware platform is upgraded from IXP2400 to IXP2800, the number of micro-engines is increased from 8 to 16. If POTs is used in this situation, there are no major changes on micro-engine programs. All we need to do is to add additional threads in the micro-engines to the free thread pool. However, HTC requires that the number of 
packet processing sub-tasks equals to that of micro-engines, which means that we need to re-design the data packet processing procedures and re-divide the data packet processing tasks [28].

Assume that most of the data packets are basically the same, and the processing time are roughly equal, HTC is more efficient than POTs during serial processing. Because in HTC model, distributed cache such as CAM and LM can be used to quickly solve the mutual exclusion problem, and the Inter-Threading-Signal mechanism is relatively simple to solve the packet ordering problem. Conversely, POTs is more efficient than HTC if differences of the processing time among data packets are relatively significant. In this case, due to strict synchronization constraint between various threads of HTC, those threads that process packets with relatively short processing time are idle, and wait for other threads taking longer processing time. This results in waste of resources and decline in processing speed. While threads in POTs run independently, there are no strict synchronization requirements.

The comparison between HTC and POTs depends on the actual situation. As described above, if the majority of the data packet and their processing time are basically the same, we can use HTC module for fast processing of data packets. If the differences are relatively large, POTs model can be used for effective processing. In addition, if we take into account simplicity of program development and scalability of the corresponding platform, POTs is a more suitable micro-engine programming model. 
For SGSN and GGSN, we proposed a comprehensive programming model which combines HTC and POTs to achieve the best processing efficiency. As shown in Figure 3-3, HTC and POTs can be combined to be responsible for front-end data packet processing tasks, while HTC is used for the back-end data packet processing tasks. We adopt HTC sub-model Context Chaining to let two micro-engines handle restructuring and receiving the packets, and then through the Reflector the packets are transmitted to the next packet processing modules which are achieved by POTs model. Once POTs completes the data packet processing, the Asynchronous-insert and Synchronous Remove (AISR) software mechanism of POTs is used to restore the sequence of the packets, and then the packets are sent to the back-end packet processing modules which are controlled by HTC sub-mode Context Chaining.

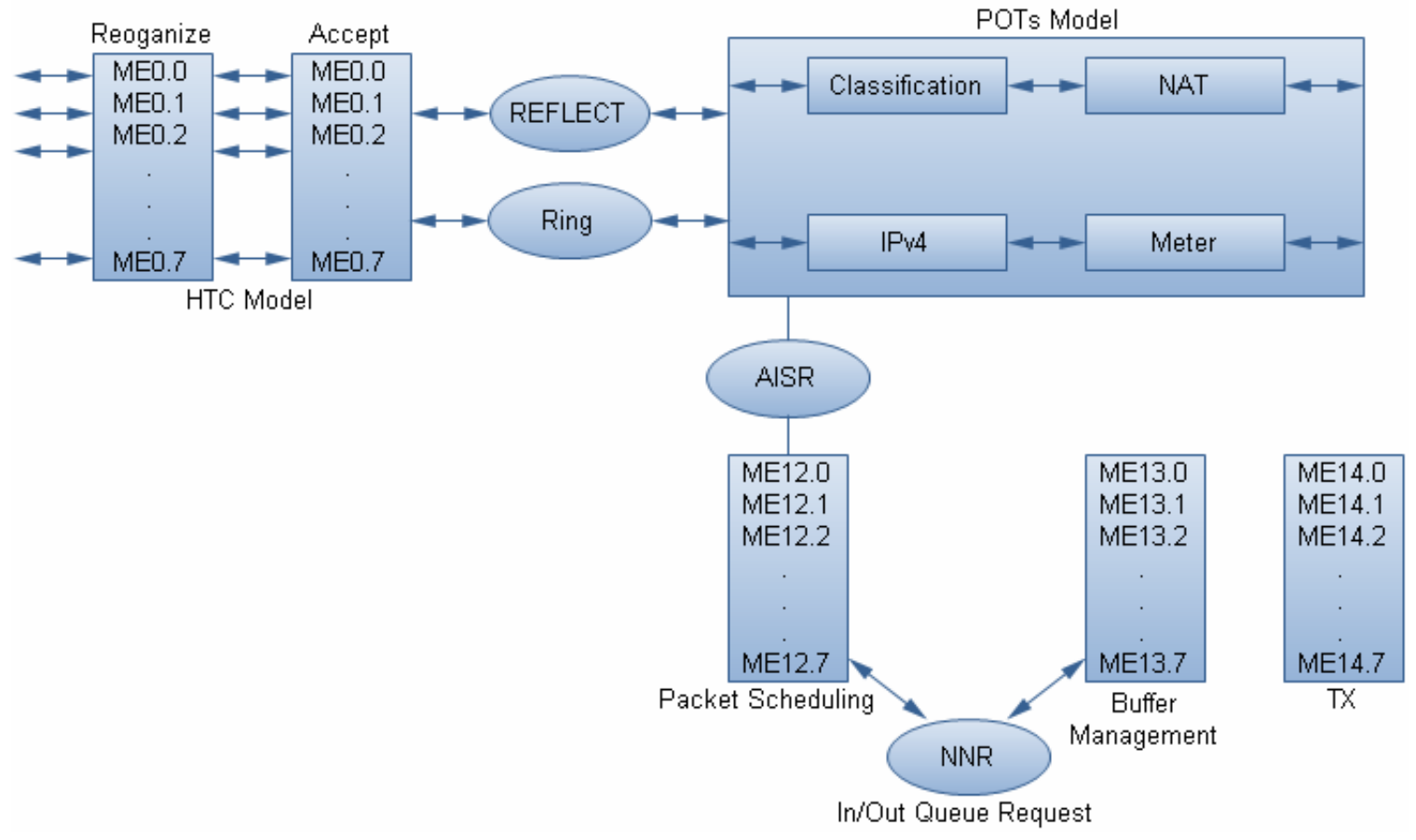

Figure 3-3 Comprehensive Programming Model Structure 


\subsection{Summary}

WCDMA QoS must be implemented on the CN element nodes GGSN and SGSN, and the data planes of SGSN and GGSN are implemented on forwarding board based on NPs. An NP has high-speed processing and flexible programming capabilities, and it is an ideal hardware platform to achieve DiffServ QoS for WCDMA CN. In gerenal, an NP is different from a general-purpose processor in its operations and development methods, so we need to better understand it in order to efficiently achieve the differentiated QoS for WCDMA CN.

In this chapter, we presented in-depth analysis of two existing programming models HTC and POTs. A comprehensive programming model was put forward to solve the sharing conflicts and packets ordering problem. This model combines HTC and POTs and maps them to different QoS management modules. This model gets the advantages of the two existing models, and is highly efficient and flexible. We use this programming model to implement DiffServ QoS for WCDMA CN. 


\section{CHAPTER 4}

\section{QUEUE MANAGEMENT ALGORITHM DESIGN}

\subsection{Queue Management Overview}

Queue management plays a significant role in the control of network transmission. It is the core mechanism to control network QoS, and the key method to solve the network congestion problem. Queue management consists of buffer management and packet scheduling. Generally the buffer management is applied at the front of a queue and cooperates with the packet scheduling to complete the queue operation $[29,30]$. When a packet arrives at the front of a queue, the buffer management decides whether to allow the packet to come into the buffer queue. From another point of view, the buffer management determines whether to drop the packet or not, so it is also known as dropping control.

\subsection{Existing Buffer Management Algorithms}

In recent years, the buffer management mechanism is a hot research area. A number of schemes have been introduced in this area. These designs adopt different control schemes for various situations. Based on basic principles of the buffer management control, the control schemes of the buffer management can be analyzed from two levels, data flow and data packet. In the data flow level if we view it from the aspect of system resource management, the buffer management needs to adopt certain resource management schemes to make a fair and effective allocation of queue buffer 
resources among flows through the network nodes. In the data packet level and viewed from the aspect of packet dropping control, the buffer management needs to adopt certain drop control schemes to decide that under what kind of circumstances a packet should be dropped, and which packet will be dropped. Considering congestion control response in an end-to-end system, the transient effects for dropping different packets may vary greatly. However, statistics of the long-term operation results indicates that the transient effect gap is minimal, and this gap can be negligible in majority of cases. In some specific circumstances, the completely shared resource management scheme can cooperate with drop schemes such as tail-drop and head-drop to reach effective control. However, in most cases, interaction between these two schemes is very large. So the design of buffer management algorithms should consider both of the two schemes to obtain better control effects $[31,32]$.

The RED algorithm [33] was proposed for active queue management (AQM) mechanism [34] and was standardized as a recommendation from IETF [35]. RED introduces congestion control to the router's queue operations, and uses early random drop scheme to smooth packet dropping in time. This algorithm can effectively reduce or even avoid the congestion in network, and solve the TCP protocol global synchronization problem. However, one concern of the RED algorithm is the stability problem, i.e., the performance of the algorithm is very sensitive to the control parameters and changes in network traffic load. During heavy flow circumstances, the performance of RED drops drastically. Since RED algorithm is based on best-effort service model, which does not consider different levels of services and different user flows, it cannot provide fairness. In 
order to improve the fairness and stability, several improved algorithms have been developed, including WRED, SRED, Adaptive-RED, FRED, RED with In/Out (RIO) $[36,37]$ etc. But these algorithms still have a lot of disadvantages. For example, a large number of studies have shown that it is difficult to find a RIO parameter setting suitable for various and changing network conditions.

\subsection{Development of the PAFD Algorithm}

This study proposed a new buffer management algorithm called PAFD (Packet Adaptive Fair Dropping). This algorithm adaptively gains balance between congestion and fairness according to cache congestion situation. When there is minor congestion, the algorithm tends to fairly drop some packets in order to ensure that all users access the system resources to their scale. For moderate congestion, the algorithm inclines to drop packets of low quality service flows by reducing its sending rate using scheduling algorithm to alleviate congestion. In severe congestion, the algorithm will tend to fairly drop packets, through the upper flow control mechanism to meet the QoS requirements, and reduces sending rate of most service flows, in order to speed up the process of easing the congestion.

In buffer management or packet scheduling algorithms, it will improve the system performance to have service flows with better transmission conditions reserved in advance. But this operation makes system resources such as buffer space and bandwidth be unfairly distributed, so that QoS of service flows with poor transmission conditions cannot be guaranteed. Packet scheduling algorithms usually use generalized processor 
sharing (GPS) as a comparative model of fairness. During the process of realization of packet scheduling algorithms based on GPS, each service flow will be assigned a static weight to show their QoS. The weight $\phi_{i}$ actually expresses the percentage of the service flow $i$ in the entire bandwidth $B . \phi_{i}$ does not change with packet scheduling algorithms, and meets

$$
\sum_{i=1}^{N} \phi_{i}=1
$$

where $N$ expresses the number of service flows in the link. And the service volume is described by

$$
g_{i}^{i n c}=\frac{\phi_{i}}{\sum_{j \in B} \phi_{j}} B
$$

where $i, j$ denotes two different service flows. In GPS based algorithms, the bandwidth allocation of different service flows meets the requirement $B_{i} / \phi_{i}=B_{j} / \phi_{j}$, where $B_{i}$ is the allocated bandwidth of the service flow $i$. By assigning a smaller weight $\phi_{\text {low }}$ to an unimportant background service flow, the weight of service flow with high priority $\phi_{h i g h}$ is much larger than $\phi_{\text {low }}$, so that the majority of the bandwidth is accessed by high-priority service flows.

\subsubsection{Algorithm Description}

In buffer management algorithms, how to control the buffer space occupation is very important [38]. Here we define 


$$
\frac{C_{i}}{W_{i}}=\frac{C_{j}}{W_{j}}
$$

where $C_{i}$ is the buffer space occupation, and $W_{i}$ expresses the synthetic weight of the service flow $i$. When the cache is full, the service flow with the largest value of $C_{i} / W_{i}$ will be dropped in order to guarantee fairness. Here the fairness is reflected in packets with different queue length [39-40]. Assume that $u_{i}$ is the weight, and $v_{i}$ is the current queue length of the service flow $i$. The synthetic weight $W_{i}$ can be calculated as described by

$$
W_{i}=\alpha \times u_{i}+(1-\alpha) \times v_{i}
$$

where $\alpha$ is the adjust parameter of the two weighting coefficients $u_{i}$ and $v_{i}$. The parameter $\alpha$ can be pre-assigned, or determined in accordance with usage of the cache. The weight $u_{i}$ is related to the service flow itself, and different service flows are assigned with different weight values. As long as the service flow is active, this factor remains unchanged. But $v_{i}$ is time varying, which reflects dropping situation of the current service flow.

Suppose a new packet $T$ arrives, then the PAFD algorithm process is described as follows:

Step 1: Check whether the remaining cache space can accommodate the packet $T$, if the remaining space is more than or equal to the length of $T$, add $T$ into the cache queue. Otherwise, drop some packets from the cache to free enough storage space. The 
decision on which packet will be dropped is given in the following steps.

Step 2: Calculate the weighting coefficients $u$ and $v$ for each service flow, and the value of the parameter $\alpha$. Then find the values of new synthetic weights $W$ for each flow according to (4-4).

Step 3: Choose the service flow with the largest weighted buffer space occupation $\left(C_{i} / W_{i}\right)$. If the service flow associated to the packet $T$ has the same value as it, then drop $T$ at the probability $P$ and returns. Otherwise, drop the head packet of the service flow with the largest weighted buffer space occupation at probability $1-P$, and add $T$ into the cache queue. Here Probability $P$ is a random number generated by the system to ensure the smoothness and stability of the process.

Step 4: Check whether the remaining space can accommodate another new packet, If the answer is yes, the packet will be transmitted into the cache. Otherwise, return to Step 3 to continuously choose and drop packets until there is sufficient space.

If all packet lengths are the same, the algorithm only needs one cycle to compare and select the service flow with the largest weighted buffer space occupation. Therefore, the time complexity of the algorithm is $O(N)$. In this case, we also need additional $4 N$ storage space to store the weights. Taking into account the limited capacity of wireless network, $N$ is usually less than 100 . So in general the algorithm's overhead on time and space complexity are not large. On the other hand, if packet lengths are different, then it is necessary to cycle Step 3 and Step 4 until the cache has enough space to accommodate the new packet. The largest cycling times is related to the ratio between the longest and 
the shortest packets. At this moment, the time complexity overhead is still small based on practices.

In Step 2, $\alpha$, a function of shared buffer, is a parameter for adjusting proportion of the two weighting coefficients $u$ and $v$. For a large value of $\alpha$, the PAFD algorithm tends to fairly select and drops packets according to the synthetic weight $W$. Otherwise, the algorithm tends to select and drop the service flow with large queue length. A reasonable value for $\alpha$ can be used to balance between fairness and performance. Here we introduce an adaptive method to determine the value of $\alpha$ based on the congestion situation of the cache, and this process does not require manual intervention.

When there is a minor congestion, the congestion can be relieved by reducing the sending rate of a small number of service flows. The number of service flows in wireless network nodes is not as many as that in the wired network. So the minor congestion can be relieved by reducing the sending rate of any one of service flows. We hope this choice is fair, to ensure that all user access to the system resources according to their weights.

When there is a moderate congestion, the congestion cannot be relieved by reducing the sending rate of any one of service flows. Reducing the rate of different service flows will produce different results. We hope to reduce the rate of service flows which are most effective to the relief of congestion. That is, the service flow which current queue length is the longest (The time that these service flow occupied the cache is also the longest). This not only improves system throughput, but also made to speeds up the congestion relief. 
When there is a severe congestion, it is obvious that reducing the sending rate of a small portion of the service flows cannot achieve the congestion relief. We may need to reduce the rate of a lot of service flows. Since the TCP has a characteristic of additive increase multiplicative decrease (AIMD), continuous drop packets from one service flow to reduce the sending rate would adversely affect the performance of the TCP flow. While the effect on relieving system congestion becomes smaller, we gradually increase the values of parameters, and the algorithm will choose service flows to drop packet fairly. On one hand, at this point the "fairness" can bring the same benefits as in the minor congestion system; on the other hand this is to avoid continuously dropping the longer queue service flow.

Congestion is measured by the system buffer space occupation rate. $\alpha$ is a parameter relevant to system congestion status and its value is between 0 to 1 . Assume that the current buffer space occupation rate is denoted by Buffer $r_{\text {cur }}$, and Buffer $r_{\text {medium }}$, Buffer $_{\min }$, and Buffer $r_{\max }$ represent threshold value of the buffer space occupation rate for moderate, minor, and severe congestion, respectively.

When Buffer $r_{\text {cur }}$ is close to Buffer ${ }_{\min }$, the system enters a state of minor congestion. The system is in a state of severe congestion when $B u f f e r_{\text {cur }}$ reaches $B u f f e r_{\max }$. The value of $\alpha$ can be determined by using linear approach, then the system will have a dramatic oscillation. Instead we use high order nonlinear or index reduction to get smooth curve of $\alpha$ as shown in Figure 4-1. 


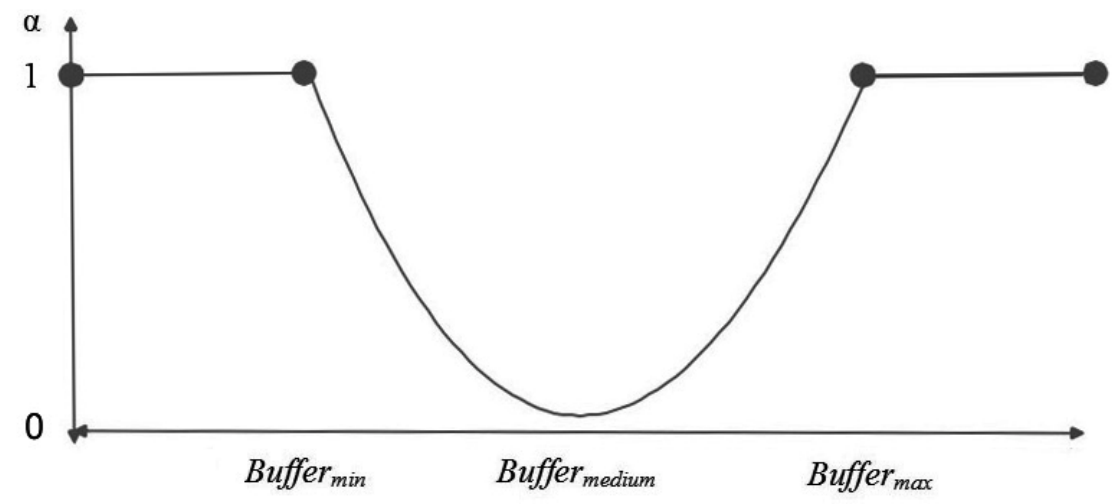

Figure 4-1 An Adaptive Curve of Parameter $\alpha$

The value of $\alpha$ can be calculated from

$$
\alpha=\left\{\begin{array}{l}
0, \quad \text { if Buffer }{ }_{\text {cur }}^{2}<\text { Buffer }{ }_{\text {min }}^{2} \\
1-\frac{\text { Buffer }_{\text {cur }}^{2}-\text { Buffer }_{\text {min }}^{2}}{\text { Buffer }_{\text {max }}^{2}-\text { Buffer }_{\text {min }}^{2}}, \text { if Buffer }{ }_{\text {min }}^{2} \leq \text { Buffer }{ }_{\text {cur }}^{2} \leq \text { Buffer }_{\text {max }}^{2} \\
1, \quad \text { if Buffer } \text { cur }^{2}<\text { Buffer } r_{\text {max }}^{2}
\end{array}\right.
$$

\subsubsection{DiffServ Support}

In the PAFD algorithm, we can adopt the DiffServ model to simplify the service flows by dividing them into high-priority services such as assurance services and lowpriority services such as best-effort services. We use the queuing method for the shared cache to set and manage the cache. When a new packet arrives at the cache, first the service flow is checked to see whether it matches the service level agreement (SLA). If it

does, then this new packet enters the corresponding queue. Otherwise, the packet is assigned to low-priority services, and then enters the low-priority queue. 
In the DiffServ model, we retain the implement process of PAFD, and only modify (4-4) into

$$
W_{i}=\left(\alpha \times u_{i}+(1-\alpha) \times v_{i}\right) \times \beta
$$

where $\beta$ is a new parameter used to adjust the fairness among service flows of different service levels.

As mentioned above, we can set the value of parameter $\alpha$ different from that shown in Figure 4-1 to satisfy different requirements. For high-priority services, the curve in Figure 4-1 is reasonable. The fairness is able to guarantee the QoS for different service flows, and is required to relief congestion quickly. For high-priority services which have no delay constraints and high fairness requirements, a higher throughput is more practical. Therefore, we can get the value of the parameter $\alpha$ for low-priority services, which is slightly less than that for high-priority services as shown in Figure 4-2.

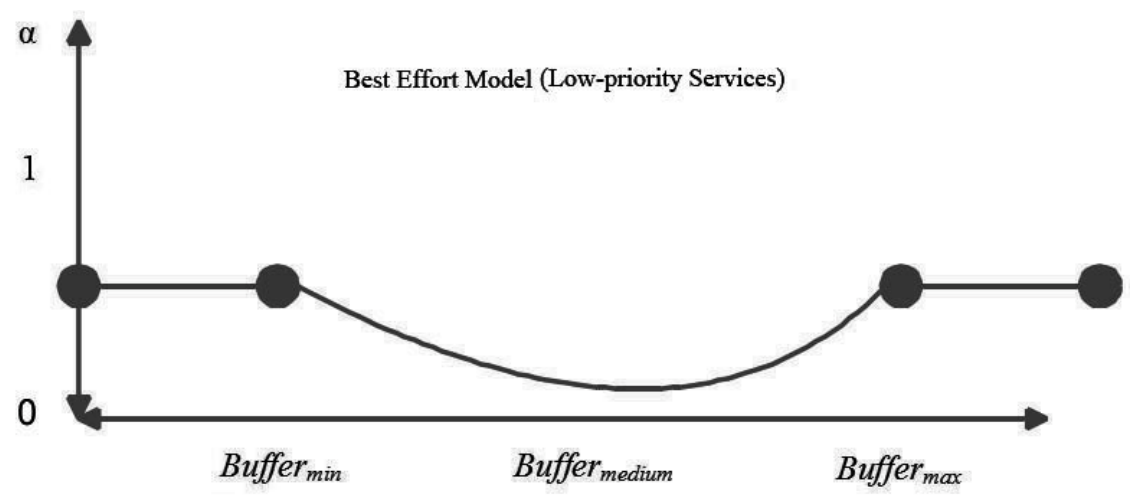

(a) The Value Curve for the Best Effort Model 


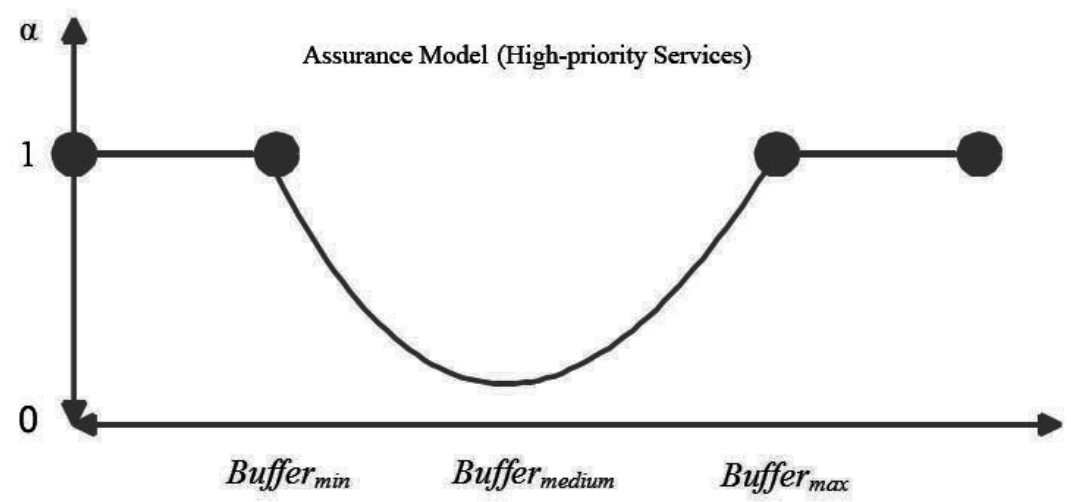

(b) The Value Curve for the Assurance Model

Figure 4-2 Values of Parameter $\alpha$ for Different Priority Services

Now we check effects of the parameter $\beta$. For high-priority services, $\beta$ is a constant with value 1 . For low-priority services, the $\beta$ is less than 1 , and influenced by the network load. When network load is low, $\beta$ equals to 1 . In this case, different level service flows have the same priority to share the network resources. As network load increases, in order to guarantee the QoS of high-priority services, low-priority services gradually give up some transmission opportunities, so the value of $\beta$ decreases. The higher network load is, the smaller the values of $\beta$ and $W$ are. Therefore, the probability of a low-priority packet being dropped is higher. Values of $\beta$ are shown below.

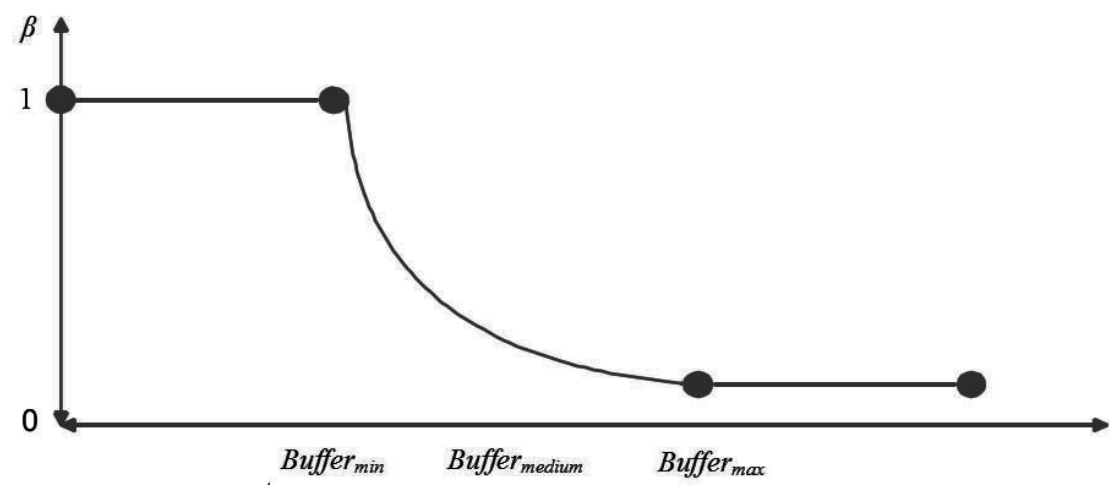

Figure 4-3 Values of Parameter $\beta$ for Different Priority Services 


\subsection{PAFD Simulation Results}

\subsubsection{Simulation for Commen Services}

We compare the PAFD algorithm with two commonly used buffer management algorithms RED and tail drop (TD). We choose two common packet scheduling algorithms Best Channel First (BCF) and Longest Queue First (LQF) to work with PAFD, RED and TD. Here the LQF uses the weighted queue length for packet scheduling. So there are 6 queue management algorithm combinations, which are PAFDBCF, PAFD-LQF, RED-BCF, RED-LQF, TD-BCF, and TD-LQF. The performance comparisons of these algorithms are carried out with respect to throughput effectiveness, average queuing delay, and fairness.

We use K1297-G20 signaling analyzer to simulate packet sending, and the operation system for K1297-G20 is Windows NT 4.0. ADLINK 6240 is used as the NP blade. Based on the simulation configuration, there are 8 different packet length configurations for the data source. They are fixed length of 64 bytes, fixed length of 65 bytes, fixed length of 128 byte, fixed length of 129 bytes, fixed length of 256 bytes, random length of 64-128 bytes, random length of 64-256 bytes, and random length of 641500 bytes.

Figure 4-4 shows that all the algorithms have similar throughputs for low network load. When the load increases, the throughput effectiveness of BCF is higher than that of other scheduling algorithms. This figure shows that PAFD-BCF provides significant 
higher throughput than the other algorithms. PAFD does not randomly drop or simply tail drop packets, but fully considers fairness and transmission conditions. In this way, service flows under poor transmission condition receive high probability of packet dropping, thus a relatively short virtual queue. When BCF is working with PAFD, the service flow under better channel transmission condition will give higher priority and result effective throughput.

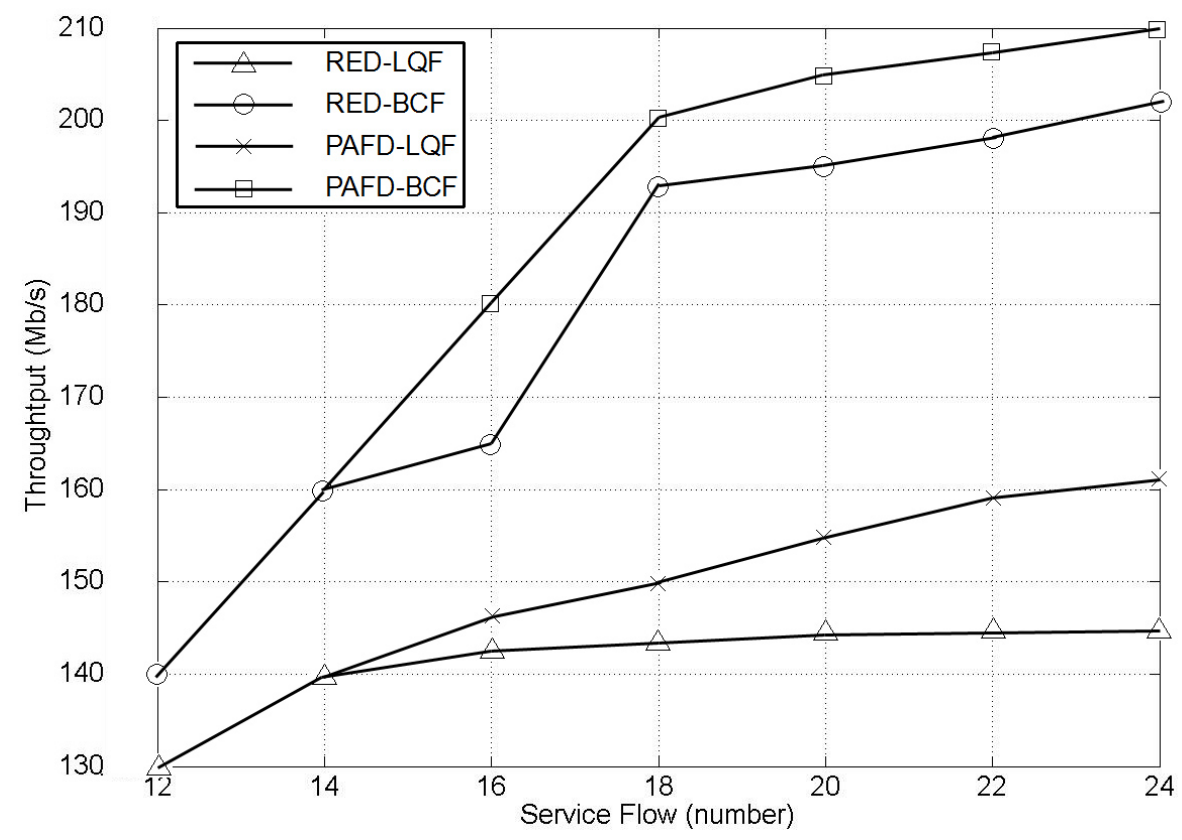

Figure 4-4 Throughputs Comparison between RED and PAFD

From Figure 4-5, we find that RED has better performance on the average queuing delay due to the early detection capability of congestion and its drop mechanism. BCF has better performance on queuing delay than that of LQF. As the load increases, the average queuing delay of PAFD first increases, then decreases. This is because PAFD does not use tail drop, and instead searches a service flow with the largest weighted buffer space occupation to drop the head packet to reduce average queuing time. 


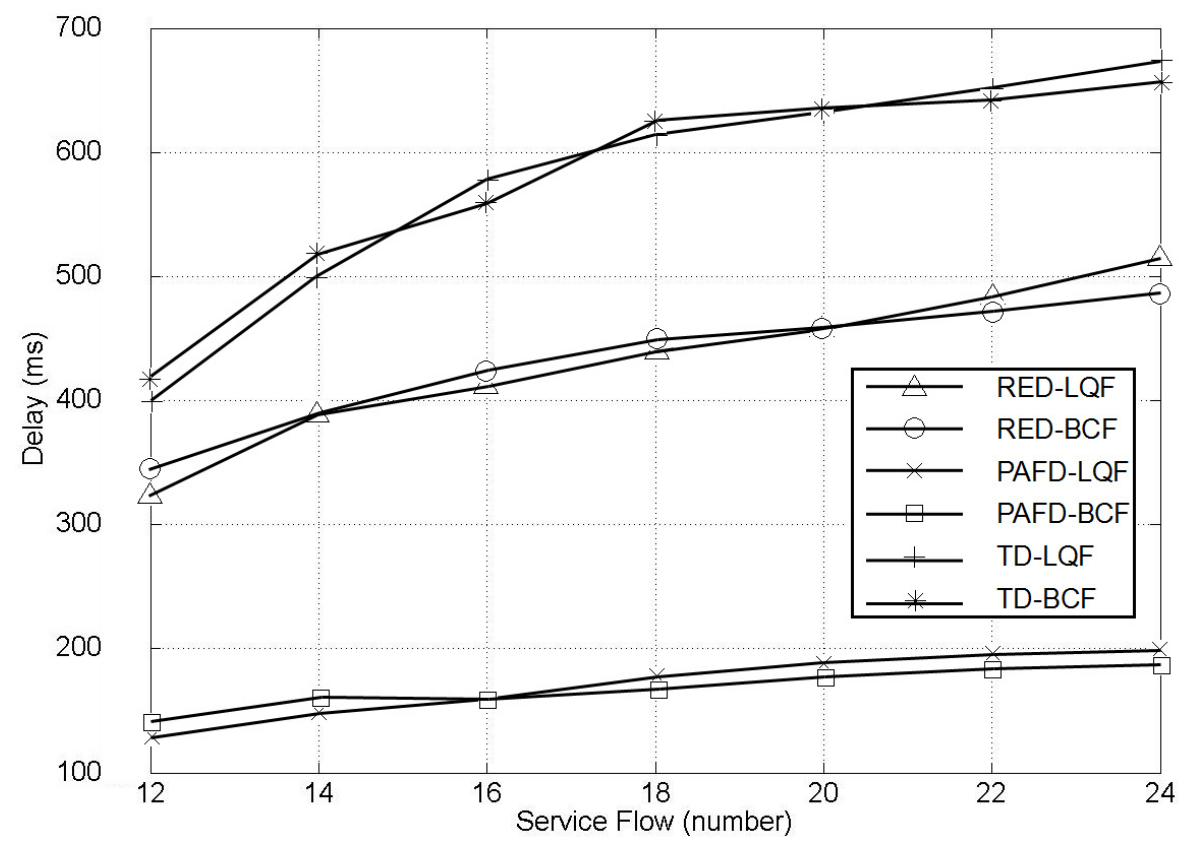

Figure 4-5 Comparison of Average Queuing Delay for TD, RED and PAFD

Both TD and RED use shared cache instead of flow queuing so that they fail to consider the fairness. Here the fairness index $F$ is given by

$$
F=\frac{\left(\sum_{1}^{N} \frac{G_{i}}{W_{i}}\right)^{2}}{N \sum_{1}^{N}\left(\frac{G_{i}}{W_{i}}\right)^{2}}
$$

where $G_{i}$ is the effective throughput of service flow $i$, and $N$ is the total number of service flows. It is not difficult to prove that $F \in(0,1)$. When $F$ has a lager value, the fairness of the system is better. If the value of $F$ equals to 1 , the system resource is completely fair. We can use (4-7) to calculate the fairness index and compare the fairness of different algorithms. In ON-OFF model with the assumption that there are 16 service flows, the ON average rate of flows $1-8$ is twice of that of $9-16$. That is, $W_{i}: W_{j}=2: 1$, where $i \in[1$, 8] and $j \in[9,16]$. Using round robin algorithms without considering $W$, we can calculate 
the reference value of fairness index $F=0.9$. Table $4-1$ gives the fairness index of TD, RED and PAFD which are combined with packet scheduling algorithms.

Table 4-1 Fairness Index Comparison of TD, RED and PAFD

\begin{tabular}{|c|c|}
\hline Algorithms & Fairness \\
\hline TD-BCF & 0.8208 \\
\hline TD-LQF & 0.9169 \\
\hline RED-BCF & 0.8839 \\
\hline RED-LQF & 0.9968 \\
\hline PAFD-BCF & 0.8915 \\
\hline PAFD-LQF & 0.9992 \\
\hline
\end{tabular}

This table indicates that the fairness index of BCF is lower when combined with TD and RED. Since PAFD takes the fairness into consideration, the fairness index of PAFD is higher than that of TD when there are congestions. The combination of PAFD and LQF has higher throughput and more fair distribution of cache and bandwidth resources. By changing the value of $\alpha$, we can conveniently balance the system performance and fairness based on the requirements.

\subsubsection{Simulation for DiffServ}

In this section we adopted the same environment as described in the previous section to test the PAFD performance based on the DiffServ model. The only difference is that half of the services are set to high-priority, and another half to low-priority.

Figures 4-6 and 4-7 show the throughput and average queuing delay of those algorithms. The only difference in these two tests is that the value of $\alpha$ for half of the 
service flows used in the latter is slightly lower than the one in the former. So the curves in Figures 4-6 and 4-7 are very similar to those shown in Figures 4-4 and 4-5.

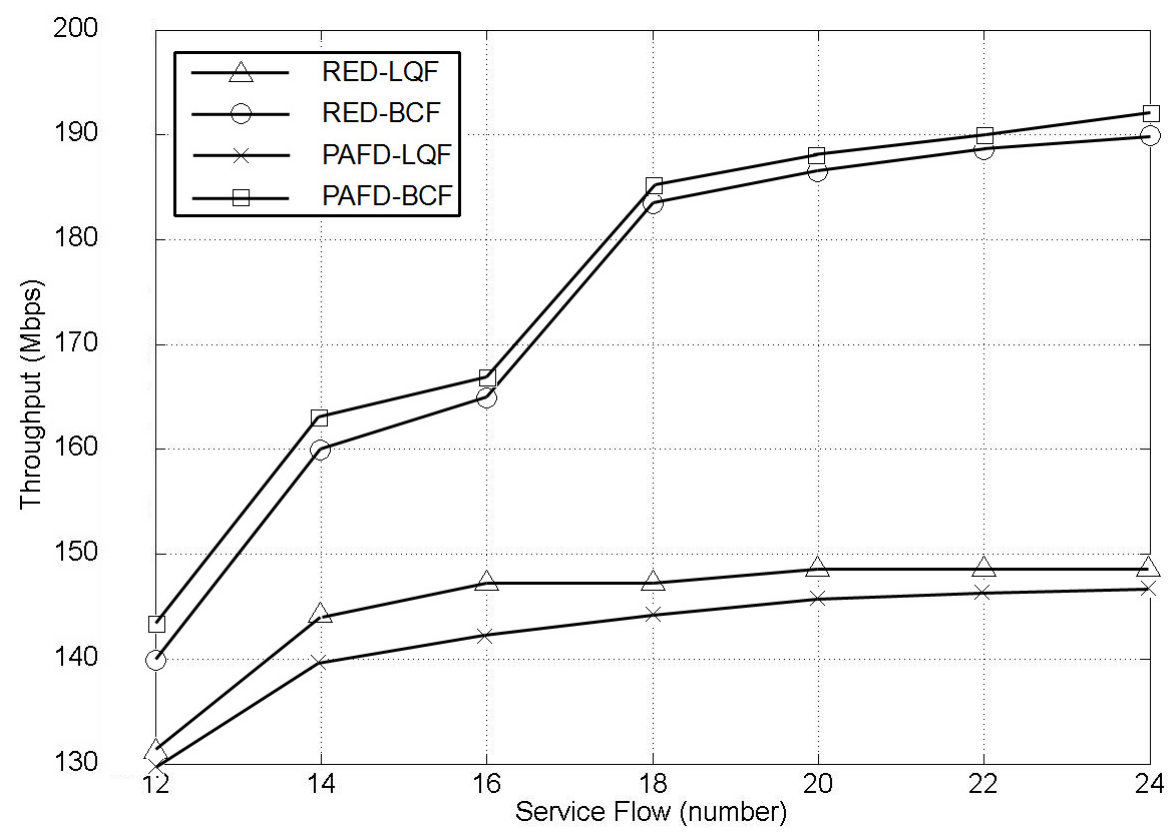

Figure 4-6 Throughputs Comparison between RED and DS-PAFD

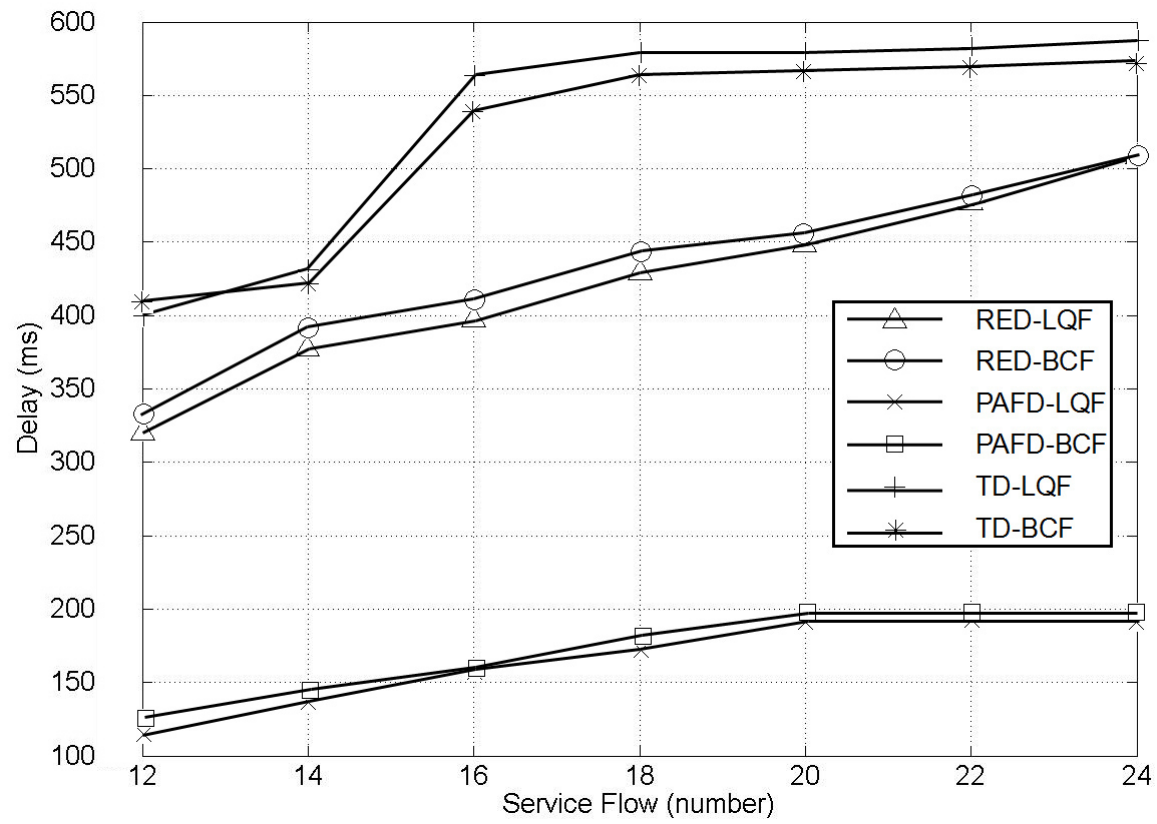

Figure 4-7 Comparison of Average Queuing Delay of RED and DS-PAFD 
Table 4-2 gives the comparison of fairness index of theses algorithms. Comparing these numbers with those shown in Table 4-1, we can draw a similar conclusion. However, the difference in values is that the fairness index of low-priority services is slightly lower than that of high-priority services as a result of different values of $\alpha$.

Table 4-2 Fairness Index Comparison between TD and DS-PAFD

\begin{tabular}{|c|c|c|}
\hline & TD-BCF & TD-LQF \\
\hline Flow & 0.8355 & 0.9271 \\
\hline & DSPAFD-BCF & DSPAFD-LQF \\
\hline High-priority Service Flow & 0.8826 & 0.9937 \\
\hline & DSPAFD-BCF & DSPAFD-LQF \\
\hline Low-priority Service Flow & 0.8342 & 0.9497 \\
\hline
\end{tabular}

As shown in Figures 4-2, 4-3, 4-6 and 4-7, when network load is light, the throughputs are similar for different priority services. This means different priority services have the same priority to share network resources. As network load increases, the throughput gradually decreases. However, even in the case of heavy load, the PAFD algorithm still allocates small portion of resources to low-priority services to meet the fairness requirement. And this operation will prevent high-priority services from fully occupying the network resources.

\subsection{Existing Packet Scheduling Algorithms}

Packet scheduling mechanism is the most important module in DiffServ agreement to implement QoS. It is also an active research area in DiffServ. It is of great practical significance to design appropriate packet scheduling mechanisms according to 
the different QoS requirements for applications. This has been the most concern for network equipment manufacturers and carriers.

Scheduling is one of the key schemes of system resource management. It is an effective mean to solve multiple services resources sharing problems. Network system resources generally consist of three parts: buffers, link bandwidth, and processor resources. The buffer management has been fully considered in hardware perspective of NPs. Link bandwidth management by packet scheduling means determining which packet is to be selected from the waiting queue and sent according to certain rules to provide better and timely services for high priority packets, so that all input service flows can follow a predetermined way to share the output link bandwidth. The main performance parameters are bandwidth allocation, latency, packet loss rate, latency jitter and so on. Figure 4-8 is a typical packet scheduling system.

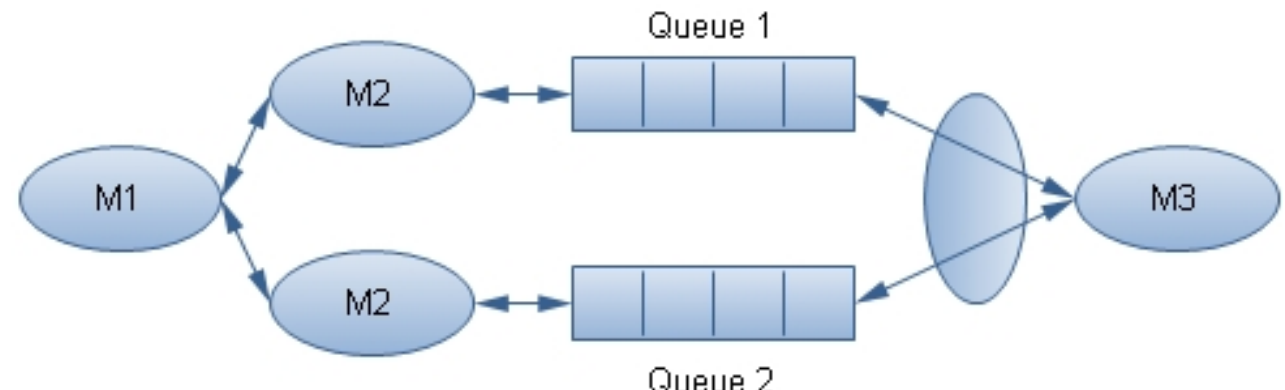

Figure 4-8 A Typical Packet Scheduling System

There is only a single queue at each output link interface for the traditional network switching equipment. As long as the underlying link has the capacity of transmitting the data, it fetches the packet from the queue as soon as possible. However, 
network environments supporting QoS require that each scheduler of switching equipment can schedule multiple queues at the same time and these queues share the output link capacity. When and how frequently to fetch the packet from each queue for transmission depends on what packet scheduling algorithms the scheduler runs. When packets in a queue are scheduled to be sent, the rest of the queues must wait. Within each queue, first-come first-served (FCFS) method can be used, and services of a packet cannot be interrupted. The basic process in packet scheduling is to make judgments based on certain queue information, and to control the frequency of various queues occupying link bandwidth, thereby affecting the latency, loss and other parameters. In general, more information is considered, more effective the algorithm is, but the complexity is also higher. Advanced service scheduling algorithms store the packets in different queues, and then compute their dynamic priorities as the control parameters.

Research on packet scheduling algorithms has been a hot topic. So far, dozens of algorithms have been proposed. They have different services rules, control objectives, and complexity. According to the working principle and control objectives, they can be divided into two groups: general processor sharing (GPS) based scheduling and roundrobin-based scheduling algorithms. Currently the GPS-based scheduling algorithms mainly are weighted fair queuing (WFQ) [41], packet fairness queuing (PFQ) [42], selfclocking fair queuing (SCFQ) [43], and virtual clock (VC). These algorithms can provide better fairness, latency characteristics, and isolation of malicious flows. However, the larger the number of queues is, the more complex these algorithms are to be implemented. Round-robin-based algorithms mainly have Weighted Round Robin 
(WRR) [44], Deficit Round Robin (DRR) [45], etc. These algorithms are to serve each divided group in turn, and the implementation is relatively simple, but they cannot provide latency guarantee on the service flow.

By analyzing some typical network applications, we can tell an appropriate scheduling algorithm should meet the following criterions: to have smaller average latency and maximum latency for low throughput applications (audio, TELNET); to provide fairness for variable bit rates (VBR) video so that its packet loss rate is within an acceptable range; to provide fairness under the changes of server processing capacity circumstances for the throughput-sensitive and flow-controlled data applications.

\subsection{Development of the PWFQ Algorithm}

The most significant characteristics that NPs differ from the general-purpose processors are multi-CPU and multi-threaded. The traditional packet scheduling algorithms cannot use these powerful parallel features, and only consider fairness of the bandwidth, rather than fairness of processor resource scheduling. Therefore, in this study a WFQ based packet scheduling algorithm called PWFQ was introduced, which can provide appropriate system guarantees on fairness, latency, and bandwidth utilization. Since WFQ algorithm cannot be directly used for processor resource scheduling and a variety of resource allocations, we design a new system virtual time function and system processing time calculation scheme in the PWFQ algorithm in order to maintain balance between bandwidth and CPU resources. 


\subsubsection{Algorithm Description}

Based on single exponential smooth (SES) forecasting method, the packet processing time is given below.

$$
F_{t+1}=a X_{t}+(1-a) F_{t}
$$

where $F_{t+1}$ is the predictive value of the next moment; $X_{t}$ is the actual value of the present

moment; $F_{t}$ is the forecasted value of current time and $a$ is a user-defined smooth coefficient $0<a<1$.

Packet processing includes packet head processing and payload processing. Packet head processing has nothing to do with the length of the packet, and payload processing is associated with the packet length. Therefore, we introduce a packet processing parameter $S F$ to characterized different packet processing.

$$
S F=\left\{\begin{array}{c}
1 \\
\frac{L_{t}+1}{L_{t}}
\end{array}\right.
$$

where $L_{t}$ is packet length at $t$ moment. We synthesize (4-8) and (4-9) to get:

$$
F_{t+1}=S F \times\left(a X_{t}+(1-a) F_{t}\right)
$$


where $L_{t+1}$ is packet length at $t+1$ moment. If the packet length changes at next moment, we introduce $r$ to represent the change of packet length. In the WFQ algorithm, the system virtual time is given by

$$
\left\{\begin{array}{l}
V(0)=0 \\
V\left(t_{j-1}+\tau\right)=V\left(t_{j-1}\right)+\frac{\tau \times r}{\sum \phi} \quad \tau<t_{j}-t_{j-1} \quad j=2,3 \ldots
\end{array}\right.
$$

The algorithm PWFQ takes into account the weight of CPU, and can be defined as

$$
\left\{\begin{array}{l}
\operatorname{Sum}_{w}(t)=\sum_{i \in B(t)} \phi_{c p u}^{i}+\phi_{b w}^{i} \\
V(t+\tau)=V(t)+\frac{\tau}{S u m_{w}(t)} \\
P P_{i}(t+\tau)=a P_{i}^{t}+(1-a) P P_{i}(t) \\
S_{i}^{k}=\max \left\{F_{i}^{k-1}, V\left(a_{i}^{k}\right)\right\} \\
E P_{i}^{k}=S F_{i} \times P P_{i}\left(a_{i}^{k}\right) \\
S F_{i}=\frac{L_{i}^{k}}{L_{i}^{p}} \\
F_{i}^{k}=S_{i}^{k}+\frac{E P_{i}^{k}}{\phi_{c p u}^{i}}+\frac{L_{i}^{k} \times r^{i}}{\phi_{b w}^{i} \times 10^{6} \times B W}
\end{array}\right.
$$

where $\phi_{c p u}^{i}$ and $\phi_{b w}^{i}$ - the CPU bandwidth and weight, $B(t)$ - set of active flows, $\operatorname{Sum}_{w}(t)$ the sum of bandwidth and CPU resources weight, $V(t)$ - system virtual time, $P_{i}^{t}$ - actual packet processing consumes at time $t, P P_{i}(t)$ - processing consumption of packet $k$ from the flow $i$ at time $t, S_{i}^{k}$ and $V\left(a_{i}{ }^{k}\right)$ - virtual start time and the virtual system time of packet 
$k$ from the flow $i, P P_{i}\left(a_{i}^{k}\right)$ - estimated processing consumption of packet $k$ from the flow $i$, $F_{i}^{k-1}$ - virtual end time of packet $k$ from the flow $i, E P_{i}^{k}$ - estimated processing time of packet $k$ from the flow $i$ in seconds, $S F_{i}$ - packet head processing parameter, $L_{i}^{k}$ - packet length of packet $k$ from the flow $i, L_{i}^{p}$ - the length of the last processed packet, $\tau$ - time difference between the two updates, $a$ - SES parameter usually with value of $0.4, r^{i}$ packet length change parameter, $B W$ - sending bandwidth (MBPS).

The scheduler maintains scheduling information of each flow such as $r^{i}, P P_{i}(t)$ and so on. After the start of scheduling, $V(t)$ and $P P_{i}(t)$ in all flows are set to 0 . When a new packet arrives, the scheduler updates the system virtual time, using (4-12) to calculate the packet virtual end time and add the packet to the corresponding flow at the same time. When an out queue request is received, the scheduler finds the packet with the smallest virtual end time and outputs it according to the head packets in each active queue. At the same time the virtual end time is updated according to (4-12).

\subsubsection{Performance Analysis}

Theorem 1: The computational complexity of PWFQ is $O(\log N)$.

PWFQ computing time mostly focuses on two tasks: computing system virtual time $V(t)$ and choosing the next first completed packet for transmission. The first task needs to sort the virtual start time of the head packet in all packets accumulation, in order to select the packet (i.e., a qualified packet). The second task needs to sort the virtual end time for all qualified packets and to select the first service packet in the actual system. In the worst case, $N$ connections are all active, at this time the computational complexity of 
the two parts are $O(\log N)$, so the computational complexity of PWFQ is the sum of the computational complexity of the above two tasks, that is $O(\log N)$.

Theorem 2: PWFQ is work-conserving.

The system virtual time at least equals to the smallest virtual start time packet of all head packets. It means at any time $t$, as long as the system has packet accumulation, there is at least one packet, whose virtual start time is equal to or less than the system virtual time. In this way PWFQ with smallest eligible virtual finish time first (SEFF) scheduling scheme is work-conserving.

Theorem 3: When we implement leak bucket control with parameters $(\delta, r)$ at the source-side, the upper bound of connection latency in the PWFQ algorithm is given by

$$
\delta_{i}^{p w f q}=\left(\frac{P_{i}^{k}}{r_{c p u}^{i}}+\frac{L_{i}^{k}}{r_{b w}^{i}}\right)^{\max }+\max \left(\left(\frac{P_{i}^{k}}{R_{c p u}}+\frac{L_{i}^{k}}{R_{b w}}\right)^{\max },\left(\frac{P_{n}^{k}}{R_{c p u}}+\frac{L_{n}^{k}}{R_{b w}}\right)^{\max }\right)
$$

PWFQ uses SEFF service scheme, so it can be proved that the system achieved by PWFQ is packet rate proportional server using the method in [46]. Meanwhile, reference [46] proved that when we implemented leaky bucket control of parameters $(\delta, r)$ at the source-side, the upper bound of the connection latency was given by

$$
\frac{\delta_{i}}{r_{i}}+\frac{l_{\max }}{r}
$$


Theorem 4: In the worst case, the fairness index of PWFQ algorithm in connection $i$ is described by

$$
c_{i, p w f q}^{k}=\frac{l_{i}^{\max }}{r_{i}}-\frac{l_{i}^{\max }}{r}+\frac{l_{\max }}{r}
$$

Proof is given as follows:

$$
\left\{\begin{array}{l}
d_{i, p w f q}^{k}-a_{i}^{k}-\left(d_{i, g p s}^{k}-a_{i}^{k}\right) \leq \frac{l_{\max }}{r} \\
d_{i, p w f q}^{k}-a_{i}^{k} \leq \frac{l_{\max }}{r}+\left(d_{i, g p s}^{k}-a_{i}^{k}\right) \leq \frac{Q_{i, g p s}^{k}\left(a_{i}^{k}\right)}{r_{i}}+\frac{l_{\max }}{r} \\
\frac{Q_{i, g p s}^{k}\left(a_{i}^{k}\right)}{r_{i}}+\frac{l_{\max }}{r} \leq \frac{Q_{i, p w f q}^{k}\left(a_{i}^{k}\right)+\left(1-\frac{\left.r_{i}\right)}{r}\right) l_{i}^{\max }}{r_{i}}+\frac{l_{\max }}{r} \\
\frac{Q_{i, p w f q}^{k}\left(a_{i}^{k}\right)+\left(1-\frac{r_{i}}{r}\right) l_{i}^{\max }}{r_{i}}+\frac{l_{\max }}{r}=\frac{Q_{i, p w f q}^{k}\left(a_{i}^{k}\right)}{r_{i}}+\frac{l_{i}^{\max }}{r_{i}}-\frac{l_{i}^{\max }}{r}+\frac{l_{\max }}{r}
\end{array}\right.
$$

where $d_{i, p w f q}^{k}, a_{i}^{k}$ represent arrival time and departure time of packet $k$ in the connection $i$, respectively.

[47] defined WFQ latency specifications. WFQ algorithm is used for CPU scheduling and packet scheduling, respectively. The latency is the sum of both.

$$
\delta_{i}^{w f q}=\frac{P_{i}^{\max }}{r_{c p u}^{i}}+\frac{P_{\max }}{R_{c p u}}+\frac{L_{i}^{\max }}{r_{b w}^{i}}+\frac{L_{\max }}{R_{b w}}
$$

where $\delta_{i}^{w f q}$ - sum of latency of scheduling CPU and bandwidth, $P_{i}^{\max }$ - maximum processing consumption of each packet in flow $i, r_{c p u}^{i}$ and $r_{b w}^{i}$ - reserved resources of 
CPU and bandwidth, $P_{\max }$ - maximum processing consumption of all flows, $R_{b w}$ and $R_{c p u}$ system bandwidth and CPU resources, $L_{i}^{\max }$ - maximum packet length of flow $i, L_{\max }$ maximum packet length of all flows.

Compared with PWFQ latency defined in (4-13), it can be seen that $\delta_{i}^{p w f q} \leq \delta_{i}^{w f q}$. The PWFQ algorithm has better latency characteristics than the WFQ. Here we can use a practical example to verify this. We assume that there are two flows: packet length of flow 1 is 100Byte and takes 9 million CPU clocks to process. Packet length of flow 2 is 900Byte and needs 1 million CPU clocks to process. The processing capacity of a communication node is $500 \mathrm{Mbyte}$, and sending link is $400 \mathrm{Kbyte}$. It can be shown that the flow 1 retained $90 \%$ of the CPU capacity and $10 \%$ of the bandwidth, while flow 2 retained $10 \%$ of the CPU capacity and $90 \%$ of the bandwidth. From the comparison of the results shown in Table 4-3, we can see the latency of PWFQ is better than WFQ.

Table 4-3 Comparison of Algorithm Results

\begin{tabular}{|c|c|c|c|c|c|c|}
\hline Flow & $P_{i}$ & $L_{i}$ & $r_{c p u}^{i}$ & $r_{b w}^{i}$ & $\delta_{i}^{\text {wfq }}$ & $\delta_{i}^{\text {wfq }}$ \\
\hline 1 & $9 \mathrm{M}$ & $100 \mathrm{~B}$ & $450 \mathrm{~B}$ & 40 & 60 & 76 \\
\hline 2 & $1 \mathrm{M}$ & $900 \mathrm{~B}$ & $50 \mathrm{~B}$ & 360 & 60 & 76 \\
\hline
\end{tabular}

\subsection{PWFQ Simulation Results}

We use 16 different flows to test the algorithm developed in this study. These flows require different $\mathrm{CPU}$ processing time. Simulation is carried out with TRANSCTOR of IXAWORKBENCH 3.0. The transmission rate is set to 50Mbps. SRAM frequency is $200 \mathrm{MHz}$. DRAM frequency is $150 \mathrm{MHz}$. PLL frequency is set to 
1200MHz. We use WORKBENCH packet generator to simulate the sending packets. The results are shown in the tables below.

Table 4-4 Flows Distribution

\begin{tabular}{|c|c|c|c|c|}
\hline $\begin{array}{c}\text { Flow } \\
\text { Number }\end{array}$ & $\begin{array}{c}\text { CPU } \\
\text { Requirements }\end{array}$ & $\begin{array}{c}\text { Bandwidth } \\
\text { Requirements }\end{array}$ & $\begin{array}{c}\text { CPU Demand } \\
\text { Instruction Cycles }\end{array}$ & Packet Length \\
\hline $1,5,9,13$ & HIGH & LOW & $2400-3600$ & $43-48$ \\
\hline $2,6,10,14$ & LOW & HIGH & $78-134$ & $120-127$ \\
\hline 7,16 & MEDIUM & MEDIUM & $1200-1800$ & $80-88$ \\
\hline $3,8,12$ & LOW & LOW & $78-134$ & $42-48$ \\
\hline $4,11,15$ & HIGH & HIGH & $2400-3600$ & $120-127$ \\
\hline
\end{tabular}

Table 4-5 Latency Comparison

\begin{tabular}{|c|c|c|c|c|c|c|}
\hline Mode & $\begin{array}{c}\text { PWFQ } \\
\text { Max } \\
\text { Latency }\end{array}$ & $\begin{array}{c}\text { PWFQ } \\
\text { Average } \\
\text { Latency }\end{array}$ & $\begin{array}{c}\text { PWFQ } \\
\text { Variance }\end{array}$ & $\begin{array}{c}\text { WFQ } \\
\text { Max } \\
\text { Latency }\end{array}$ & $\begin{array}{c}\text { WFQ } \\
\text { Average } \\
\text { Latency }\end{array}$ & $\begin{array}{c}\text { WFQ } \\
\text { Variance }\end{array}$ \\
\hline $\begin{array}{c}\text { High CPU } \\
\text { High Bandwidth }\end{array}$ & 1.05 & 0.68 & 0.13 & 1.1 & 0.79 & 0.13 \\
\hline $\begin{array}{c}\text { High CPU } \\
\text { Low Bandwidth }\end{array}$ & 1.02 & 0.69 & 0.13 & 1.1 & 0.77 & 0.15 \\
\hline $\begin{array}{c}\text { Low CPU } \\
\text { High Bandwidth }\end{array}$ & 0.82 & 0.58 & 0.1 & 1.09 & 0.70 & 0.13 \\
\hline $\begin{array}{c}\text { Low CPU } \\
\text { Low Bandwidth }\end{array}$ & 0.79 & 0.55 & 0.09 & 1.0 & 0.68 & 0.13 \\
\hline $\begin{array}{c}\text { Medium CPU } \\
\text { Medium Bandwidth }\end{array}$ & 0.96 & 0.68 & 0.12 & 1.08 & 0.79 & 0.13 \\
\hline
\end{tabular}

From these two tables, we can see that the latency of PWFQ has obvious improvement over WFQ. In an average case, i.e., the high CPU and high bandwidth, the latency reduction is $13 \%$; for the low bandwidth and high $\mathrm{CPU}$, the latency decreases by $12 \%$; for the low-bandwidth and low CPU, the latency is reduced by $21 \%$. 
The PWFQ algorithm also has smaller variance, because the consumption of comprehensive scheduling of packets in the flow is less than or equals to that of scheduling them separately.

\subsection{Summary}

Queue management algorithm is the key scheme to achieve network QoS control. It is an important element of network resources management. By controlling different types of packets access and use the link bandwidth, different data flows get different levels of services. It is necessary to ensure high speed scheduling, latency QoS and fair access guarantee. It is also very important to consider the comprehensive performance of the algorithms while pursuing simplicity and easy implementation.

In this chapter we discussed the queue management algorithms from the buffer management and packet scheduling aspects. Based on the hardware features of NPs, PAFD and PWFQ algorithms were proposed. The PAFD algorithm takes into account the impact of packet transmission environment. It can adaptively gain balance between the congestion and fairness based on the congestion status of cache. The experiment results showed that the system achieved better balance between throughput and fairness after applying the algorithm. Packet scheduling algorithm PWFQ gives full consideration of the characteristics of NPs. It adds CPU scheduling based on the traditional WFQ scheduling algorithm. The simulation measurements showed that this algorithm achieved better latency guarantee than the WFQ. 


\section{CHAPTER 5}

\section{PACKET CLASSIFICATION ALGORITHM DESIGN}

\subsection{Introduction}

Packet classification mechanism is mainly used in classifiers and the regulators of NPs [48]. Its role is to ensure that input data flows accords with the TCA. This mechanism classifies the flows to certain behavior aggregate, and tags them correspondingly as packets. The classifier follows the rules specified in TCA and assigns packets to different classes in the group according to some domains in header. Then they will be handed over to the appropriate regulator module for further processing.

Generally the classification process is carried out at the communications subnetwork nodes or routers. The routers are divided into either flow or non-flow identification routers. Flow identification routers track the transmission and conduct similar processing on the packets of the same flow. Non-flow identification routers process each incoming packet separately. Main processing steps on each packet in the flow identification router are: routing table lookup, packet classification, packets special treatment such as discarding unauthorized packets, and exchange scheduling.

\subsection{Existing Packet Classification Algorithms}

With in-depth research of network service traffic characteristics, the implementation of regulator has become more mature. The usual practice is to use token 
bucket and the leaky bucket algorithms, and other appropriate combination such as the single rate Three Color Marker (srTCM) and two rate Three Color Marker (trTCM) [49]. Recently a time sliding window based algorithm called time sliding window Three Color Marker (tswTCM) has been proposed. This algorithm uses the rate estimator to measure the average rate in the time sliding window, and then uses this rate as the basis of marker [50].

From the implementation point of view, these algorithms can be divided into two kinds. One is hardware based algorithm that can be fully implemented by hardware, and the other is software based algorithm for software implementation. Hardware based algorithms such as ternary CAMs algorithm [51], use the parallelism feature of hardware to conduct packet classification. By doing so, the classification process is fast, but the available dimension is small, the scalability is poor, and the cost is high. Also some operators cannot be directly supported. And the utilization of the memory array may not be very efficient. They are not practical for PC-based routers. On the other hand, software based algorithms generally provide specific dimension classification capability, especially the common one-dimensional and two-dimensional classification algorithms. For example, set pruning tries, grid of tree [52], and Area-based quad-tree (AQT) algorithms [53] are two-dimensional classification algorithms. However, algorithms that can provide support for high-dimensional classification may not well meet the requirements on space complexity and time complexity. For example, hierarchical cuttings algorithm [54] require large space in the worst case, and for some other algorithms, such as bit vector search algorithm [55] and back tracking algorithm, the 
classifying speed is slower. Cache-based [56] approach also does not work very well in practice due to the low hit rate and smaller flow duration.

\subsection{Development of the CBNPs Algorithm}

Most of the current packet classification algorithms are based on tree structure, but the programming of IXP2400 does not support recursive calls, and access of the tree structure is very complex. So we proposed a multidimensional packet classification algorithm called CBNPs which uses linear data structure.

\subsubsection{Algorithm Description}

The CBNPs algorithm includes two parts: preprocessing and classification. The preprocessing is mainly preparations for simplifying and accelerating the classification operation. The main function of classification is fast index processing on sorting fields mapped by packets.

Given the rules database $R D$, assuming it has $n$ of $K$-dimensional (in the case $K$ =3) rules, represented as $r_{1}, \ldots, r_{n}$, respectively. Here $r_{i, j}$ represents the value of $j$ dimensional field of the rule $r_{i}$. The preprocessing of CBNPs algorithm is as follows:

1) According to the definition of tuple space, we can find the corresponding tuple to the $n$-rule set. Assume that there are $m$ tuples in total, and $1<m<n$. Then $t_{1}, \ldots, t_{n}$ represent the rules contained in the tuple $t$ which are stored in the hash table.

2) Mapping $r_{i, j}$ to the $j$-axis to form $2 n+1$ disjoint intervals on the $j$-axis. 
3) For each interval $e(1<e<2 n+1)$ on the $j$-axis, we assign an $m$-bit vector $B_{e}$. Each bit in the vector represents one tuple. These bits are sorted by tuple priority. If and only if there exists $r \in t_{i}$ and the projection of $r$ on the $j$-axis coveres $e$, we can set the bit corresponding to $t$ in $B_{e}$ to 1 , otherwise it is 0 .

Suppose a packet $P$ arrives, then the classification process through the rule search is as follows:

1) First, in each dimension $j$, we use binary search or other search algorithms to find the interval corresponding to $P$, and thus get the bit vector $B_{j}$ corresponding to $P$ in the $j$-dimension.

2) For all the bit vectors $B_{1}, \ldots, B_{n}$, we can get the tuple corresponding to the bit with the first value of 1 in $B$. And this tuple is the tuple $t$ with the highest priority including the packet $P$.

3) In the hash table corresponding to the tuple $t$, we get the rule $r$ matched with $P$ by a memory access.

\subsubsection{Performance Analysis}

In the preprocessing of the CBNPs algorithm, we assume that there are a total of $n$ rules and $m$ corresponding tuples. In a general case, $m<<n$, which means the space occupation of bit vectors is very low. Since hundreds of rules are in the rule base of NPs, if we use tuples instead of the rule bases, space occupation reduction of each bit vector is considerable. 


\subsubsection{Space Complexity Analysis}

As discussed before, $n$ rules projection on the $j$-axis produce a maximum of $2 n+1$ mutual non-covered intervals. If an $m$-bit vector is assigned to each interval, we have the following relation

$$
C_{s}=K \times(2 n+1) \times m
$$

where $C_{s}$ is the space used by all bit vectors, and $K$ represents the dimension. Space complexity of the CBNPs algorithm is $O(m n)$, which is the same as that of the parallel algorithm. In the worst case, $m=n$. But $m<<n$ on the average, this greatly reduces space complexity.

The concept of tuple space is derived through the observation of actual rule bases. It is a heuristic solution to solve packet classification issues by using the tuple space. This solution can guarantee less space complexity in the average conditions, so space complexity of the CBNPs algorithm is much less severe than the parallel algorithm.

\subsubsection{Time Complexity Analysis}

If the number of bits in a bit vector is reduced, the amount of memory access required to read the vector will also be reduced. Here we have

$$
C_{t}=K \times m / w
$$


where $C_{t}$ represents the required times for memory accesses if all bit vectors are read, and $w$ is the word length of one memory access. In the CBNPs algorithm, vectors can be read on each dimension in parallel, the same as bit parallel algorithms. In addition, one memory access is required to access the corresponding hash tables to find the matching rules, but this access time can be ignored. Therefore, the total time complexity is $O(t+m n / w)$. Again, the worst case happens when $m=n$, and this indicates the time complexity of the CBNPs is the same as that of parallel algorithms. Since in most cases the CBNPs algorithm satisfies the condition of $m / w<<n / w$, the time complexity of the CBNPs algorithm is greatly improved over parallel algorithms.

\subsubsection{Algorithm Implementation and Optimization}

From the classification process, it can be seen that when the number of partial matching or field matching is 1 , the classification process is completed. So we conduct a direct matching operation to make sure the rule $r$ really matches the packet $P$. In fact, with the assumption that the number of part matching or fields matching rules is $k$, if the cost to directly match the $k$ rules is less than the cost to search the rest of the rules, then we can conduct direct $k$ times of matching operations. In this way, the classification process can be accomplished faster. Here's a how to determine the maximum value $K$ that satisfies the above criteria $k$. Upper bound of $K$ is hardware-related. The value of $K$ may be different if the algorithm is running on different NPs. The CPU instructions used in this algorithm are mainly the following: access memory operation including SRAM access and SDRAM access where accessing time is set to $M_{\text {sram }}$ and $M_{\text {sdram }}$; comparison operation where the cost is set to $C$; logic operation where the cost is set to $A$; testing 
instructions of location where the first bit is 1 in a 16-bit word, for which the cost is set to findset. If $k$ filtering rules are left after searching a number of sorting databases, whether to conduct direct matching or continue to search depends on the following inequality:

$$
K\left(4 M_{\text {sram }}+5 C\right)<M_{\text {sdram }}+A+4 \text { findset }+\left(4 M_{\text {sram }}+5 C\right)
$$

where $4 M_{\text {sram }}$ means using four SRAM memory accesses to fetch a rule $r$, and $5 C$ expresses using five times operations to compare the five fields. $M_{\text {sdram }}$ uses one SDRAM access to fetch the index which matches the packet fields. $A$ is the new preparation set matching the new fields by performing bitwise operation to the previous preparation set. The term 4findset represents using four instructions to find the first bit corresponding to the rule $r$ matched by the fields in the original rule base. The term $4 M_{\text {sram }}+5 C$ determines whether the rule $r$ really matches the packet $P$. Here we can rewrite (5-3) into (5-4).

$$
K<\frac{M_{\text {sdram }}+A+4 \text { findset }+\left(4 M_{\text {sram }}+5 C\right)}{\left(4 M_{\text {sram }}+5 C\right)}
$$

Once a machine is decided to run the algorithm, the cost of executing each instruction in the formula can be determined. So is the value of $K$. When the number of rules matched with the entered packets is less than or equals to $K$, the matching operation can be directly conducted without further search operation. This will accelerate the classification process. 


\subsection{Simulation Results}

The micro engines of IXP2400 provide chained SDRAM memory accesses to ensure continuous high-speed access to SDRAM. And the bus arbiter gives higher priority to memory accesses from chained SDRAM than other micro-engines to ensure that instructions are continuously sent to the SDRAM unit. SDRAM chained access is completed by adding chain-ref options to the SDRAM instructions. The instruction parameter settings are determined based on the number of rules when the program is run.

If the number of rules is less than or equals to 64 , we can run the SDRAM instructions without parameters while taking a certain field from the fields sorting library. The format is as follows: sdram [sdram_cmd, $\$ \$$ sdram_xfer-reg, source-opl, source-opt], where [sdram_cmd] means the instruction operations; [\$sdram_xfer-reg] is the name of register which stores the read data; [source-opl] and [source-opt] calculate the memory start address where SDRAM is read and written. By default, one memory word can be read each time, so 64 rules can be taken each time.

When the number of rules is between 65 and 256 , we need to add ref-count parameter into the SDRAM instructions. This parameter represents how many 4-word length storage units are continuously accessed during each memory access. The SDRAM instructions set ref-count value between 1 and 4 , so the number of rules is between 65 and 256 .

When the number of rules is greater than 256, the SDRAM instructions with indirect-ref parameter conduct continuous access of the SDRAM. In the edge routers, the 
rules are few in the rule base. Only about $0.7 \%$ of the rule bases have more than 1000 rules, and the average number of rules in most cases is about 50 [50].

In this simulation, we adopt K1297 signaling analyzer to simulate packet sending, and the operation system for K1297-G20 is Windows NT 4.0. We use 128 rules to implement this algorithm on Intel IXP2400, and randomly generate these rules according to the following strategies:

1) $25 \%$ of the rules belong to the same prefix. For example, 166.111.*** is used to simulate the LAN environment.

2) $50 \%$ of the rules are based on TCP, and $45 \%$ of the rules are based on UDP, because most of the rules are for TCP / UDP protocol.

3) $40 \%$ of the rules are for port 80 (WWW), and $20 \%$ of the rules are for ports 20 and 21 (FTP).

In the simulation, we randomly produce 1000 different types of data flows with $100 \%$ hits. Each packet length is 64-byte. The rule set has a total of 397 rules, in which source or destination address field of 126 rules are non-precise bit-string. We have four defined non-precise bit strings, which are intranet, extranet and two regions. We also define 24 types of services, including a definition of the source port number, and two definitions of a particular destination port number within the range. In addition, to test the algorithm performance of cases with even larger rule set, we randomly generate 2,000, 
$10,000,20,000,30,000,50,000$ and 100,000 rules. Evaluation results have been listed in

Table 5-1, which shows the average results obtained from 16 times of program running.

Table 5-1 Measured Results from Real Application

\begin{tabular}{|c|c|}
\hline Number of Rules & Throughput(Rule/ms) \\
\hline 397 & 3,660 \\
\hline 2,000 & 3,660 \\
\hline 10,000 & 3,620 \\
\hline 20,000 & 3,540 \\
\hline 30,000 & 3,440 \\
\hline 50,000 & 3,300 \\
\hline 100,000 & 3,200 \\
\hline
\end{tabular}

From the results, it can be seen that the algorithm fully shows the parallel nature of NPs. It is better than the existing packet classification algorithms in code complexity and overall performance.

\subsection{Summary}

This chapter analyzed the existing packet classification algorithms and compared their performances. Combined with the characteristics of all kinds of classification rules, a new multi-dimensional packet classification algorithm based on the NP IXP2400 was proposed, namely CBNPs. This algorithm reduces the number of classification rules and classification domain width to accelerate the classification and it also use parallel tuple search technology to accelerate the speed of classification. Especially, it uses the multithreaded concurrency features of NPs. The algorithm has high-speed, multi-dimensional and scalable features. The comprehensive performance of this algorithm is better than the existing packet classification algorithms. 


\section{CHAPTER 6}

\section{REALIZATION OF DIFFSERV QOS FOR WCDMA CN}

\subsection{Introduction}

According to the rate of flow, QoS can be divided into edge and core QoS switching. Edge QoS switching is complex, and its basic functions are packet reorganization, packet classification, flow regulation, queue scheduling, buffer management, routing and switching, storage and forwarding. These complex processes need gigabit switching speed. Therefore, we use two IXP2400 NPs in our design. In addition, we equip appropriate Ethernet interface hardware at the input and output of IXP2400, and set up memory such as SDRAM and SRAM for the storage external interface controller. How much memory space is needed depends on complexity of the application. Core QoS switching is relatively simple, and it only needs to perform simple tasks such as switching routing, queue scheduling and shaping instead of complex classification and aggregation.

In WCDMA CN, GGSN is the edge switching equipment, and SGSN is the core switching equipment. We mainly expounds the QoS switching technology on GGSN, and this edge QoS switching can still be used in the core switching. However, it is more effective when used in the edge switching. The QoS switching topology diagram in the network is shown in Figure 6-1. 


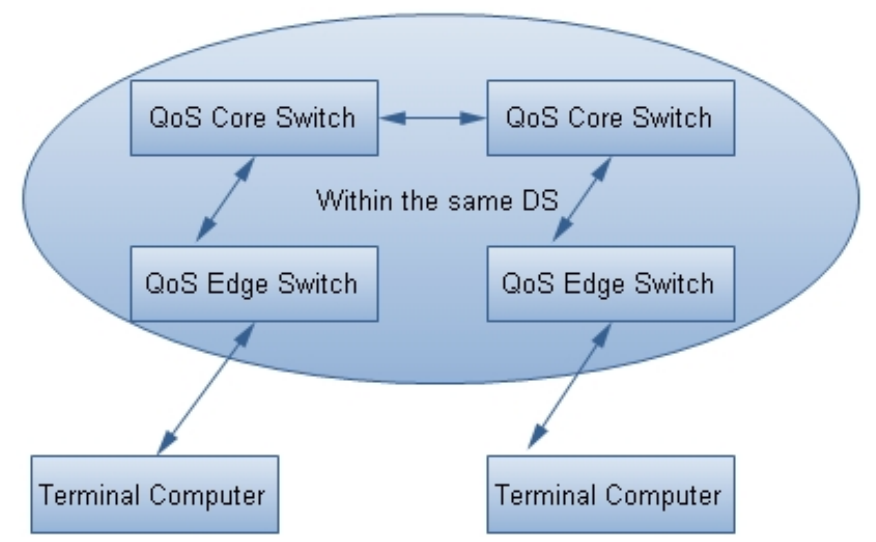

Figure 6-1 Positions of QoS Switching in a Network

From Figure 6-1, we can see that in the same DS domain, when the edge GGSN receives IP data packets, it executes packet classification and flow shaping on these data packets at the edge, and then sent them to the SGSN core switches. In WCDMA CN, these classified IP packets are aggregated, forwarded, and then sent to the QoS switch at the other end through other bearer network, and then forwarded to the clients. If two clients in different DS domains are switching data, the end-to-end QoS cannot be guaranteed. In this case, we need a QoS standard which can be established by a third party, thereby to form a unified QoS. The situations beyond the DS domain are not discussed here. Based on the programming model and algorithms proposed in the previous chapters, we have the following hardware and software design.

\subsection{Hardware Design of WCDMA QoS Based on IXP2400}

Because IXP2400 is the second-generation NP developed by Intel, its hardware design implementation is relatively simple. The hardware architecture diagram is shown in Figure 6-2. 


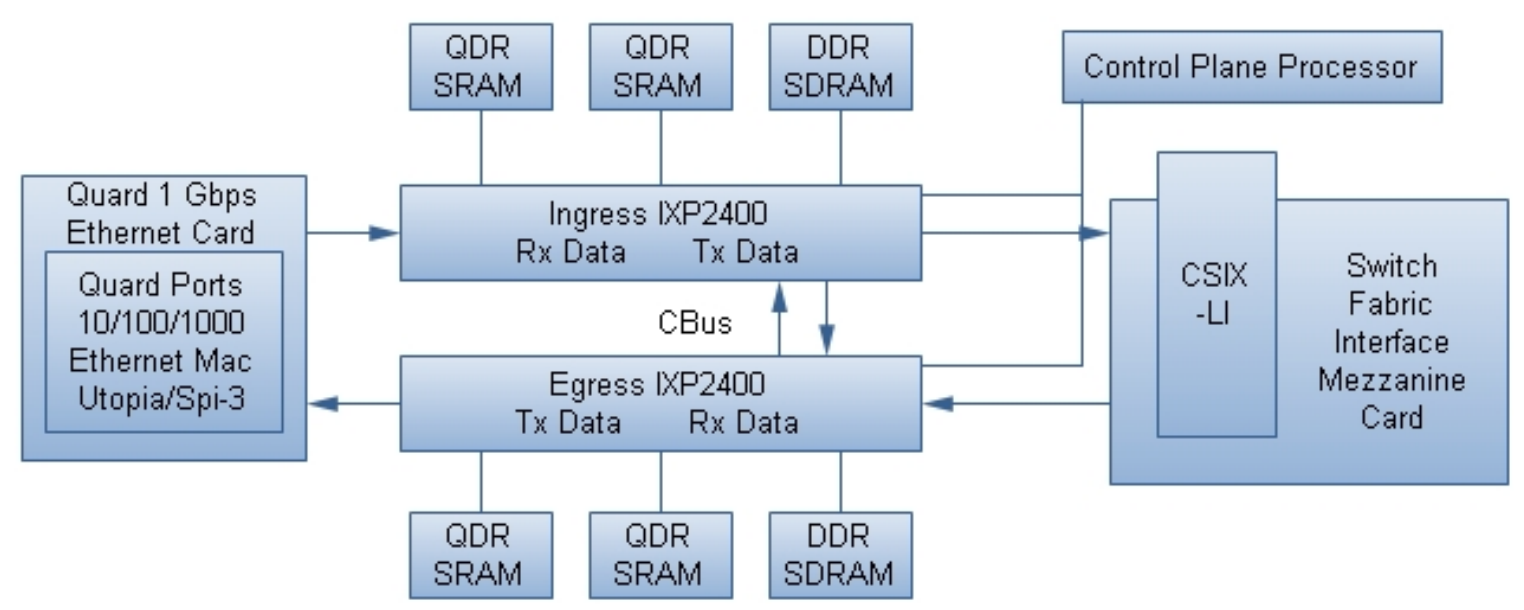

Figure 6-2 Hardware Architecture of DiffServ QoS

Figure 6-2 is a hardware configuration of SGSN switching structure. It can be seen that we use two IXP2400 NPs and a number of external devices. This hardware architecture adopts store-forward method to forward data packets, and uses queues technologies to achieve QoS. After the processing of Ingress and Egress processors, the classified and reorganized data packets are forwarded. The design and implementation of the major hardware modules are as follows:

1) Ingress IXP2400 and Egress IXP2400: We assign different tasks to each hardware thread. These tasks can be various functional modules such as packet reorganization, packet classification, flow regulation and queue management. Based on this design, the Ingresses in the two IXP2400 NPs are used to process the Ethernet IP packets which enter the switch, and the Egresses are used to process CSIX packets transmitted from Switch Fabric Interface.

2) Switch Fabric Interface: This interface is a high-speed data switching platform and sub-port which connects several devices. It directly forwards data packets coming 
from a certain port to another specified port without any processing. The supported protocols and standards include CSIX-LIB, SPI3, POS-2, Utopia Layer 1/2/3 devices, as well as providing an interface for flow control.

3) Quad 1G Ethernet Cards: They support 4 Ethernet cards with the speed of 1Gbps. And also provide Ethernet input and output for NPs.

4) SRAM and DRAM: Their role is providing the data received from interfaces with storage space so that they can be used by micro-engines and other modules.

5) Control Plane Processor: Generally the entire QoS system layer architecture is divided into data, control and management plane. Data plane provides high-speed forwarding and general data packets processing. In IXP2400, data plane consists of fast path such as micro-engines which process most of packets and slow path such as Intel Xscale core which deals with a small amount of exceptions packets. Control plane processes the protocol messages. It is also responsible for setting up, configuring and updating the routing tables and data set in data plane. Management plane is responsible for system configuration, collection, reporting status, stop and start the input or message of user application. Generally management plane is implemented in a graphical interface to display and access information from the users.

\subsection{Software Design of WCDMA QoS Based on IXP2400}

Based on the above hardware architecture, we design the software architecture diagram as below: 


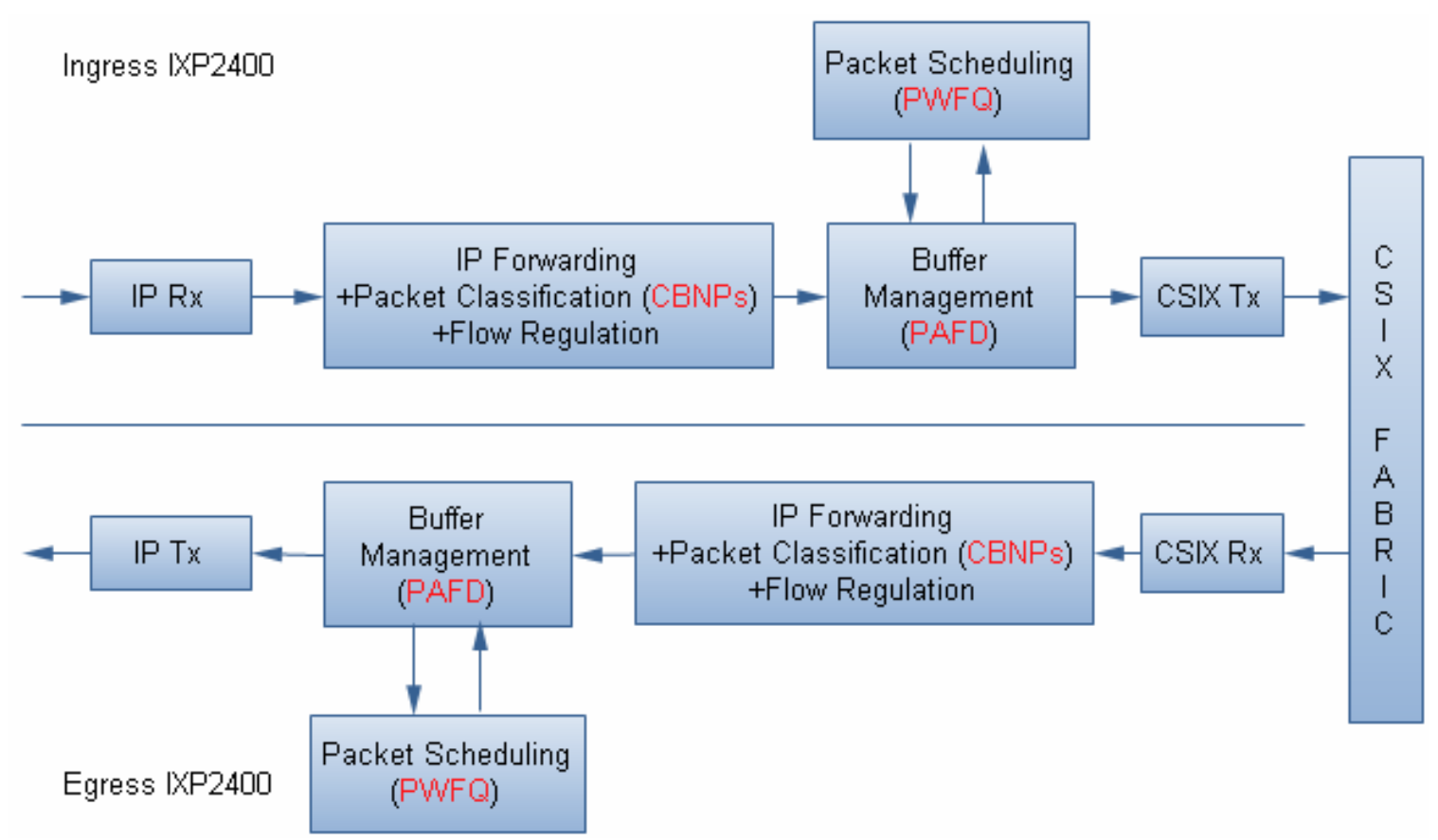

Figure 6-3 Software Architecture of DiffServ QoS

From Figure 6-3, we can clearly see the IP data flow. First the received packets are stored in DRAM, while the data header is stored in the SRAM which retains a pointer pointing to the corresponding data packet in DRAM. Then a series of QoS modules such as packet classification, flow regulation, buffer management and packet scheduling are applied. Finally, the packets are forwarded by IP forwarding module. The functional requirements and codes of the main modules in Figure 6-3 are summarized as follows:

1) IP Rx receives Ethernet packets from the RBuf which is the data buffer of IXP2400. The packets are stored in DRAM after they are reorganized. The header information of these packets is stored in Buffer Descriptor which has a pointer pointing to the corresponding packets in DRAM. Then the data packets are transmitted to the next module. 
The code of the Rx module (file: rx.uc) is below:

\#macro rx_init[]

\#macro xbuf_alloc[str_xbuf_name, NUMBER_OF_REGS, RW_INDICATOR]

\#macro pkt_rx[]

\#macro dl_sink[wxfer_prefix, req_sig, sig_nask]

\#macro xbuf_free(str_xbuf_name]

2) IP forwarding + Packet classification + Flow regulation module receives packets from IP Rx module, and then forward them after classification and regulation. The code of this module (file: fwder_classify.uc) is

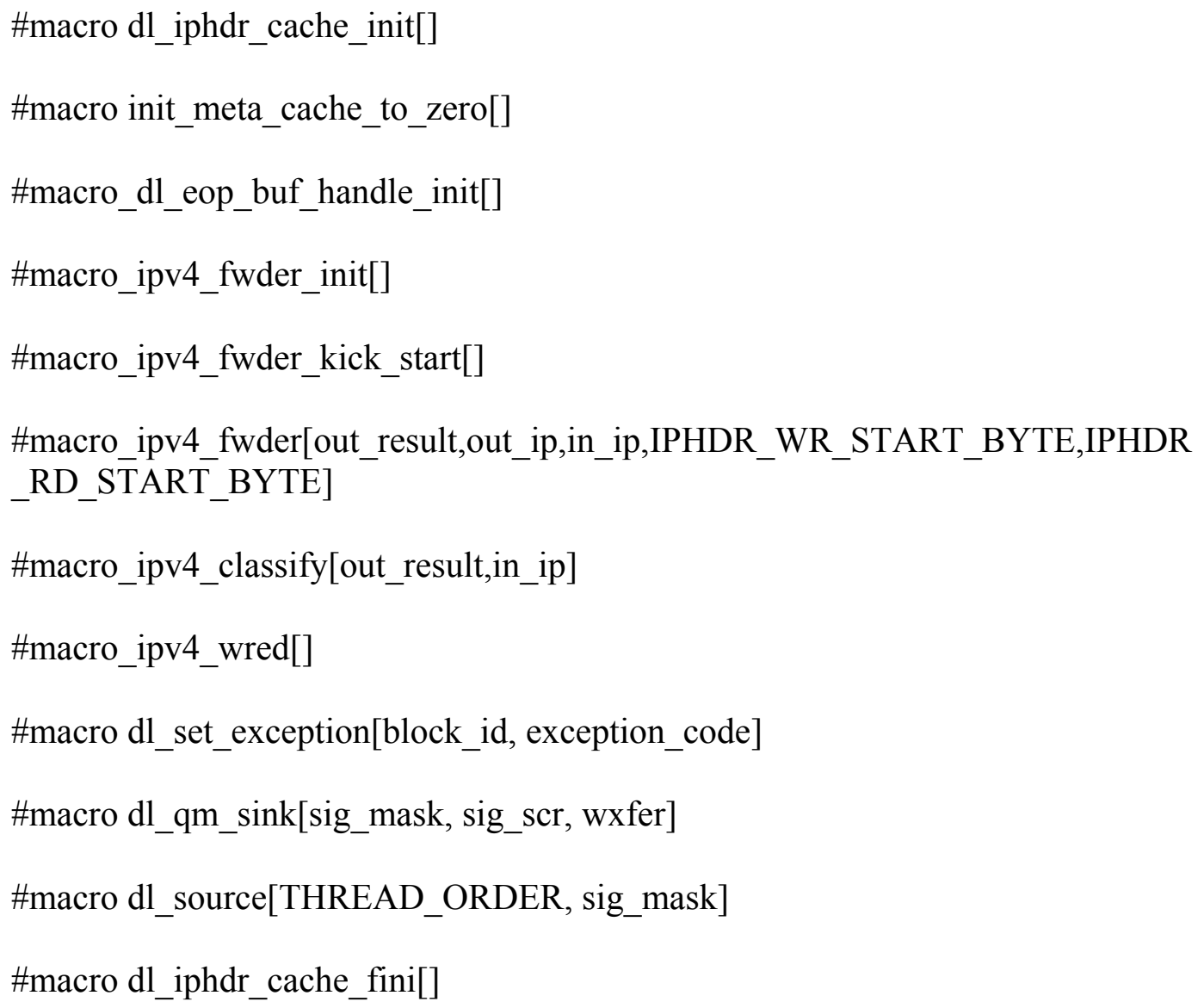


3) Buffer management and packet scheduling module is also known as queue management module. First packet scheduling module receives the information from the previous module, and then informs buffer management module to do enqueue processing which adopts PAFD algorithm. When the CSIX module is idle, packet scheduling module informs buffer management module execute dequeue processing which uses PWFQ algorithm. The buffer management code (file: qm_code_in.uc and qm_code_out.uc) is

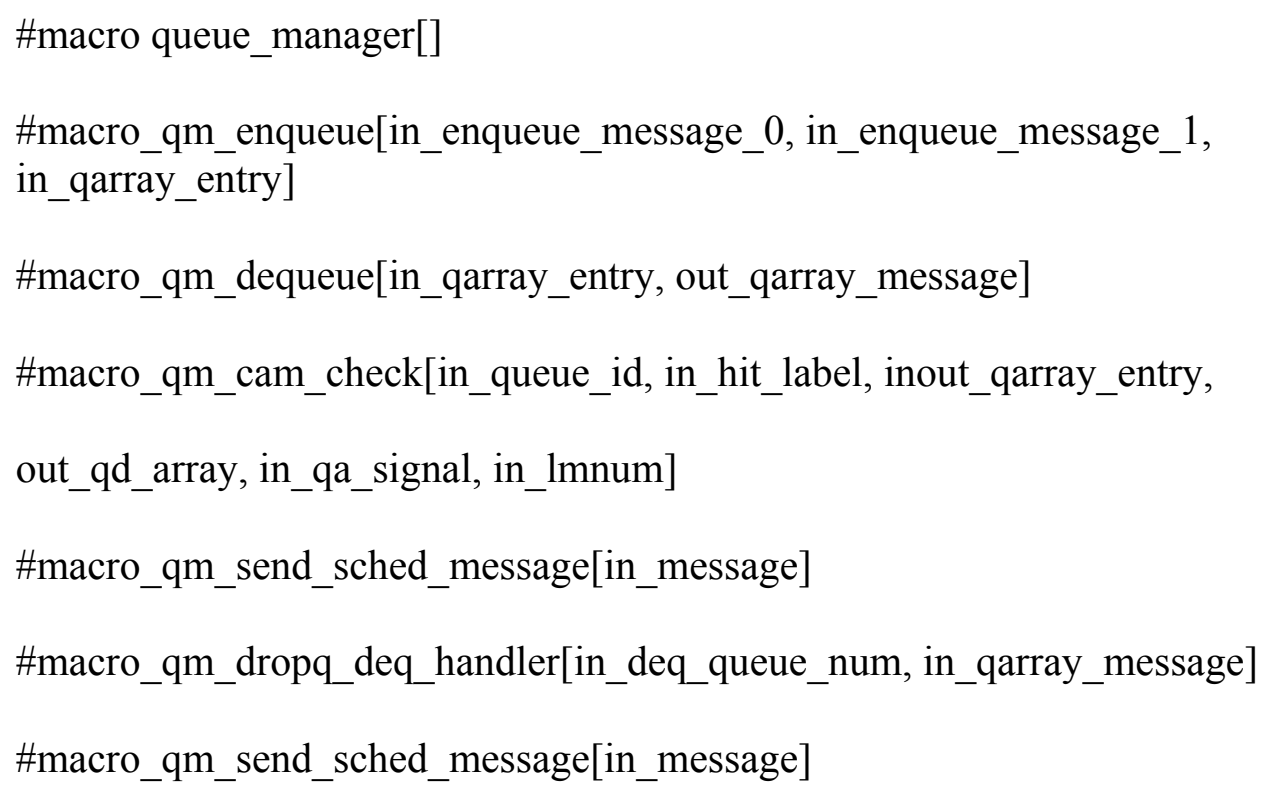

And the packet scheduling code (file: Scheduler_in. uc and Scheduler_out.uc) is

\#macro Scheduler[ring, DeqMessage, minusTwo, queueLmBase,

groupMask, deqSignal, waitSignal]

\#macro QM_MessageHandler[]

\#macro HandleFlowControl[]

\#macro ReadTbufsTransmitted[]

\#macro GetCreditIncrement[CreditIncrement, QueueId] 
4) CSIX Tx adds the related information including classification and Buffer handle in the DRAM to the dequeue packets from queue management module and sends them to CSIX Fabric to enter the next IXP2400. CSIX Rx receives the packets and other information from CSIX Fabric through RBuf and sends them to the Egress IXP2400. It is the reverse process of CSIX Tx. The code of CSIX Tx and CSIX Rx (file: csix_tx.uc and csix_rx.uc) is given below:

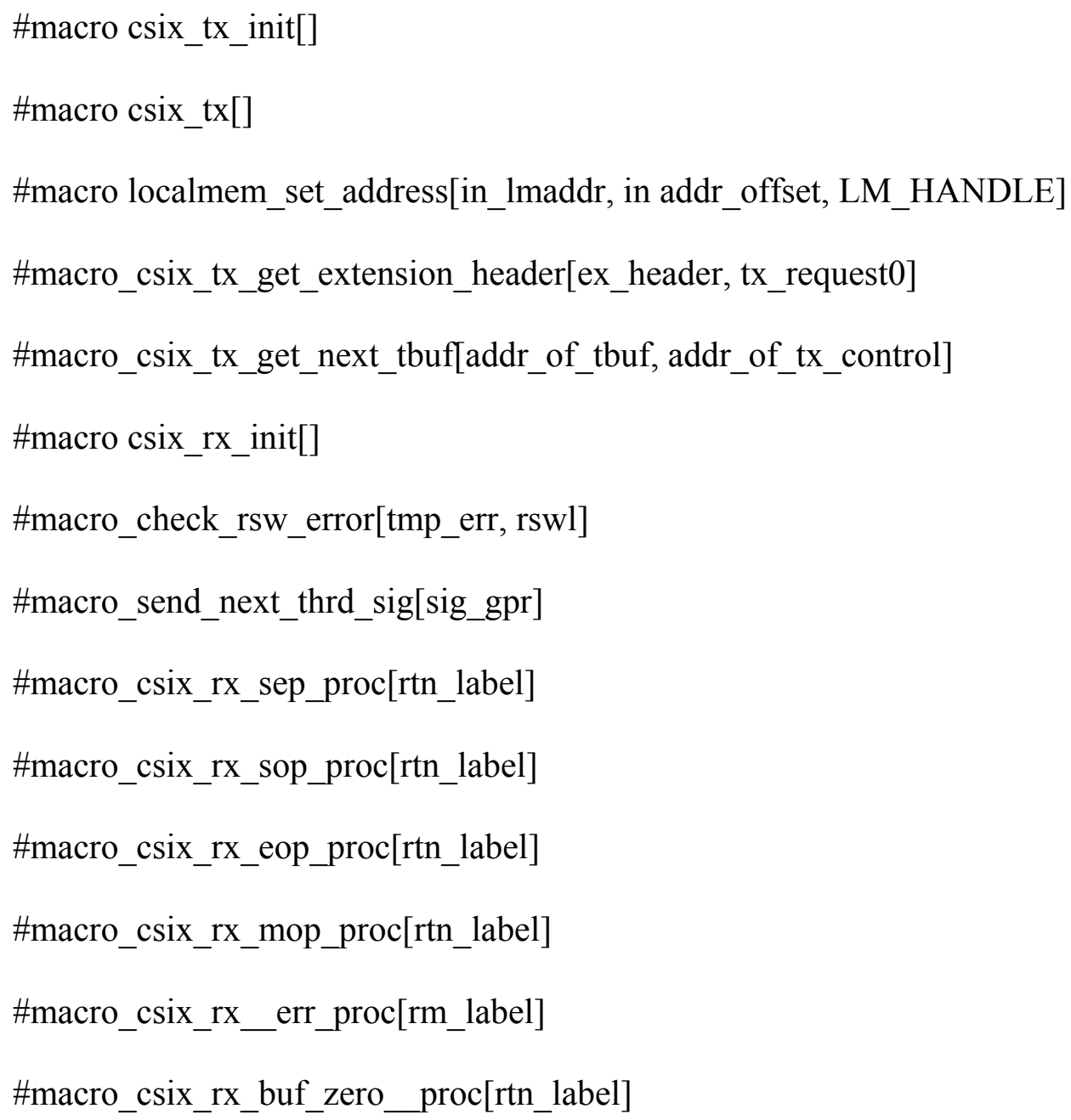

5) IP Tx executes the reverse process of receiving IP data packets, and the realization method is similar to IP Rx. The IP Tx Module code (file: tx.uc) is listed below: 
tx_init[]

$\operatorname{tx}[]$

\subsection{Simulation Results}

In this session, we used different simulation environments to test the DiffServ model and the proposed system, respectively.

\subsubsection{DiffServ Test}

We adopt the following network structure to test the DiffServ model,

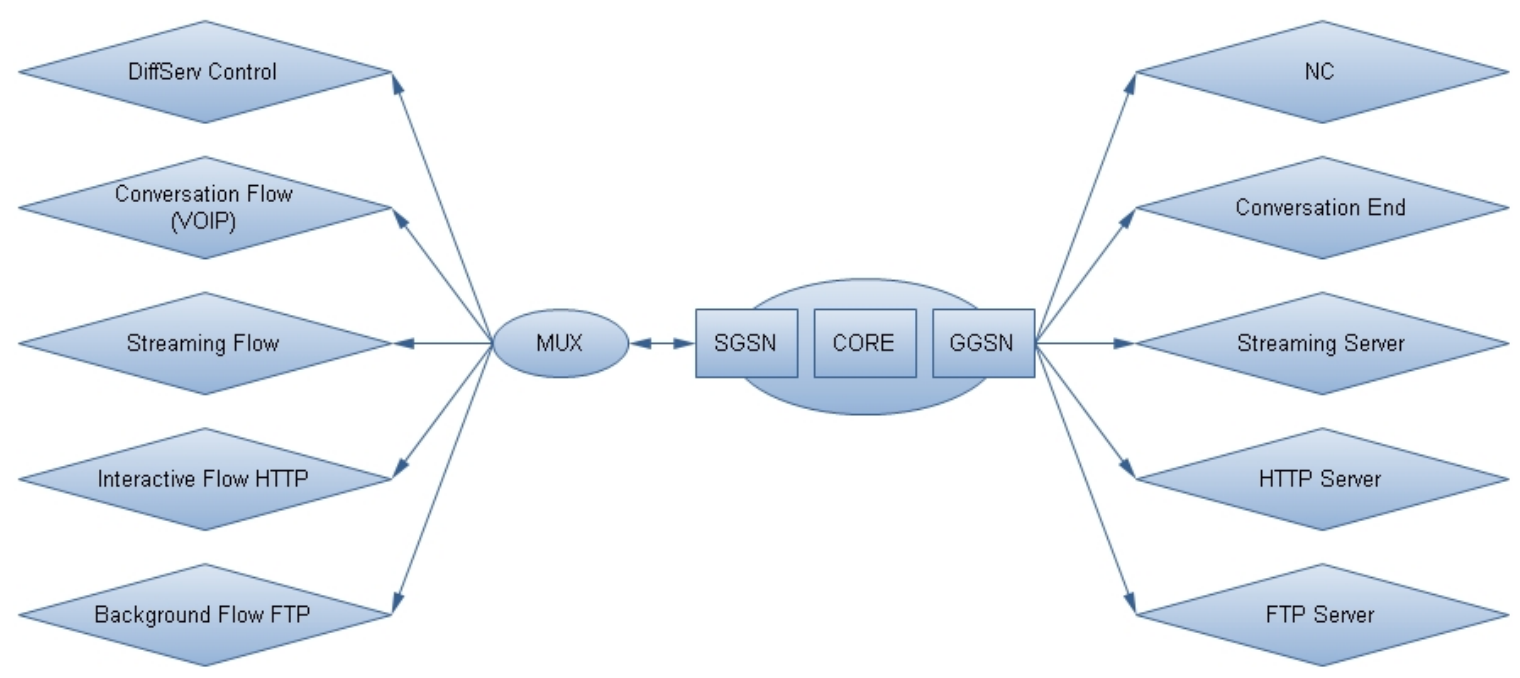

Figure 6-4 DiffServ Test Structure

In order to verify the QoS assurance mechanism of WCDMA based on the DiffServ model, we tested the CN of the entire network, to obtain the differences of QoS assurance for different service flows using the DiffServ model. 


\subsubsection{Test Environment}

1) Testing Tools

Currently the methods based on the network performance test are: simulation test and script-based tool testing. Tektronix K1297-G20 signaling analyzer showed as below can provide very flexible hardware modular and software platforms for monitoring, modeling and simulating network services that developed for $3 \mathrm{G}$ standard. It provides a wide choice of interface modules and software. It can be used to test mobile communications networks, the $\mathrm{CN}$ and most of the interfaces that access the networks. K1297-G20 implements monitoring, modeling and simulation, including the network elements simulation and related tests.

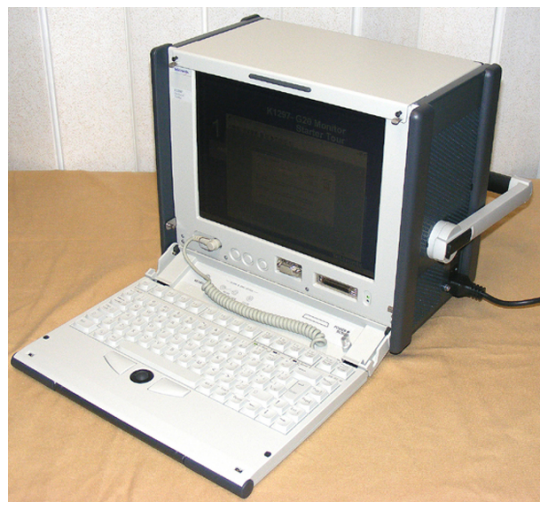

(a)

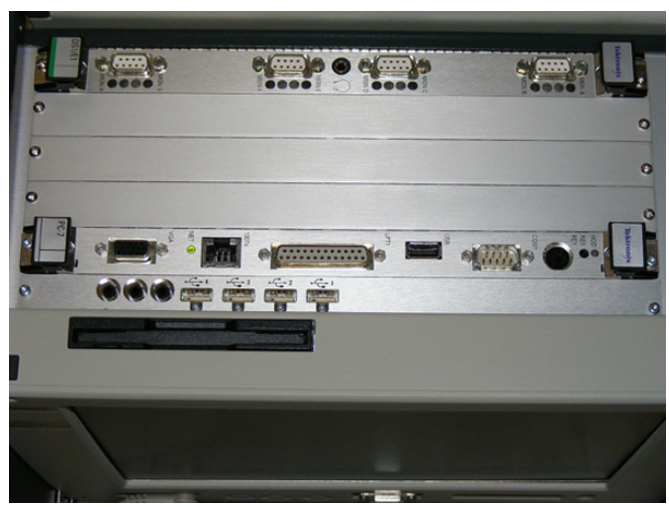

(b)

Figure 6-5 (a) K1297-G20 Signaling Analyzer (b) The Interface of K1297

2) K1297-G20 System Configurations

- Operating System: WinNT 4.0 
- Processor: $68040(33 \mathrm{MHz})$

- Central Unit: PC-AT compatible VME bus PC with 8M memory CPU

- Interface: VGA (built-in flat panel or external monitor) SCSI Ethernet

- Expansion slots: 3 free ISA slot, length: 3X2/3AT

- Mass storage: one 3/2 inch floppy disk drive (1.44MB capacity) one SCSI hard disk drive

- External CD-ROM drive

- Monitor: Built-in active TFT display, 1024×768 pixels resolution, external display port (VGA)

The system configuration Status is as below:

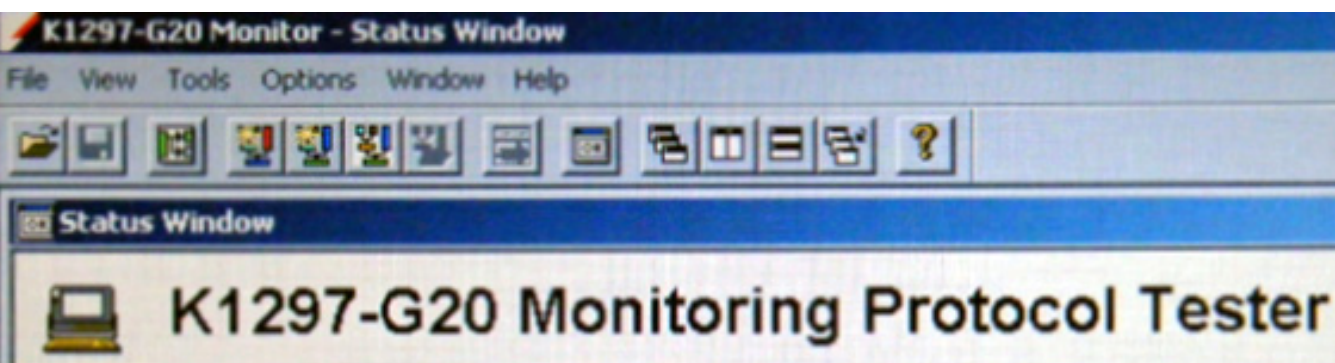

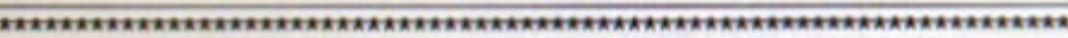

* Start of K1297-G20 Monitor application on 2/23/2010 6:56:55 PM

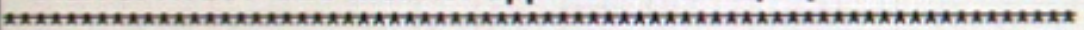

[2/23/2010 6:56:49 PM] PRIMO [1]: 32 MB RAM, S/N 00000A6D6787

[2/23/2010 6:56:51 PM] PRIMO [1]: Interface drivers installed

[2/23/2010 6:57:00 PM] PRIM0 [1]: Management Software installed

[2/23/2010 6:57:05 PM] PFIM0 [1]: Pipeline elements installed

[2/23/2010 6:57:06 PM] PRIMO [1]: Pipeline Manager started

[2/23/2010 6:57:06 PM] PRIMO [1]: Hard- and Software OK

[2/23/2010 6:57:06 PM] PRIM0 [1]: boot OK

[2/23/2010 6:57:06 PM] Number of Link Licences found: 0

Figure 6-6 K1297-G20 System Configuration Status 


\section{3) Test Design}

The network topology used in the test process is shown in Figure 6-5. In the test process, K1297-G20 is used to simulate the users and all kinds of service flows sent by the wireless access part. As showed in Figure 6-7, we can choose UMTS/WCDMA type to test the DiffServ model. The service flow types can be set in the K1297-G20 simulated user messages. i.e. the traffic class option can be set to one of the conversation, stream, interaction, and background type according to its encoding rules. In the test process the first K1297-G20 is used to send the FTP service flow of background type. The second K1297-G20 is used to send traffic flows of conversation type which is also called Voice over IP (VOIP). We choose 6240 blade of Adlink Technology as the NP blade.

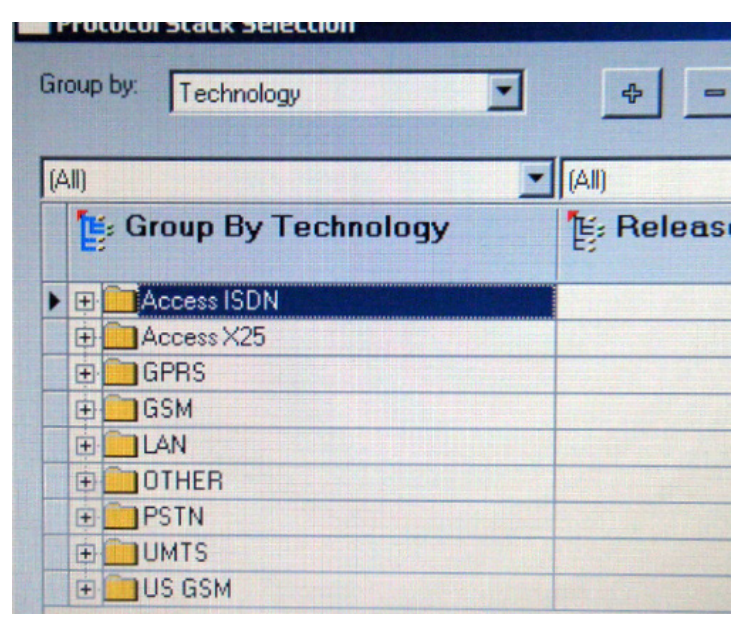

(a)

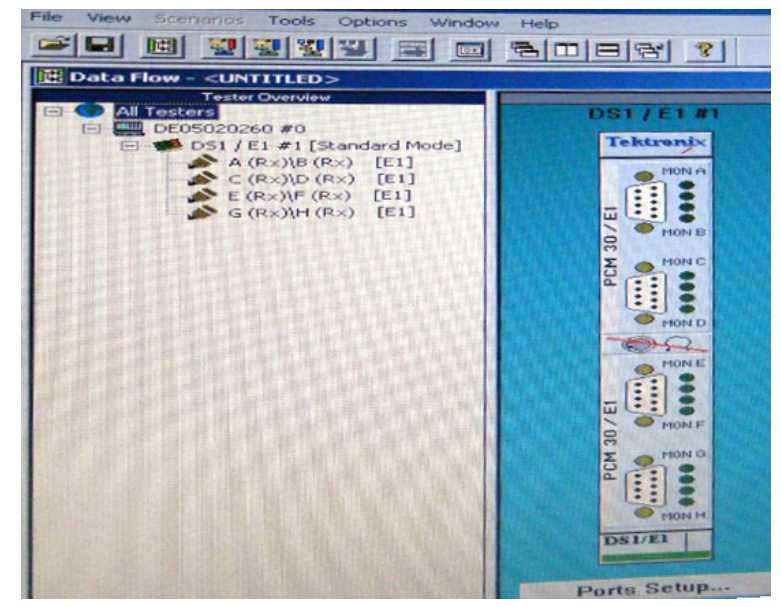

(b)

Figure 6-7 (a) Test Type Options (b) Data Flow Setup

The test is mainly for the following two parameters:

- Delay time: This parameter reflects the QoS. The shorter the time delay is, the better QoS to the user requests is. 
- Throughput: This parameter best reflects the performance of mobile networks. The larger the throughput is, the more user requests are accepted and serviced in a unit time.

\subsubsection{Test Cases}

In the network architecture built for this test, according to the set proportion, FTP data flow is added to the first K1297-G20, the other three kinds of WCDMA service flows are added to the second K1297-G20 in turns. Then slowly increase the network traffic of the second K1297-G20, and observe the delay time for this service flow. Then the proportion of the service flows through the two K1297-G20 analyzers are changed to observe the influence of the background and the other three WCDMA service flows under different proportions. In this dissertation the comparison result of VOIP and FTP are given.

1) Test Case 1

- $\quad$ VOIP traffic is set to $10 \mathrm{Mbps}$;

- $\quad$ FTP flow is set to $10 \mathrm{Mbps}$;

- VOIP traffic: FTP flow = 5: 5;

- Objective: To test the delay and throughput in the case that no differentiated services are applied.

2) Test Case 2

- $\quad$ VOTP flow is set to $2 \mathrm{Mbps}$; 
- FTP flow is set to 10Mbps;

- VOIP traffic: FTP flow = 1: 5;

- Objective: To test the delay and throughput.

3) Test Case 3

- VOIP traffic is set to $10 \mathrm{Mbps}$;

- FTP traffic is set to $10 \mathrm{Mbps}$;

- VOIP traffic: FTP flow = 1: 1;

- Objective: To test the delay and throughput.

\subsubsection{Test Results and Analysis}

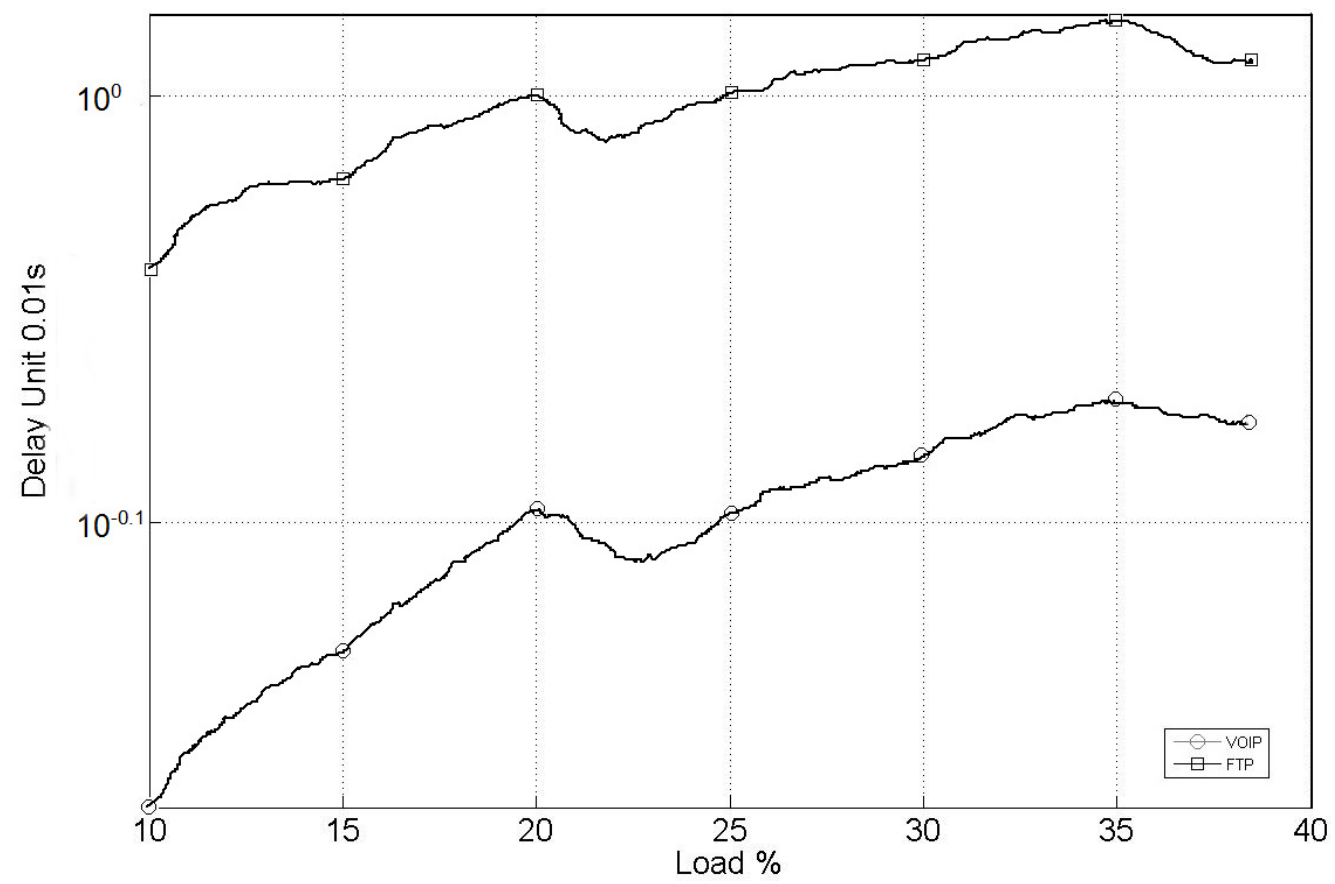

Figure 6-8 Delay for Test Case 1 


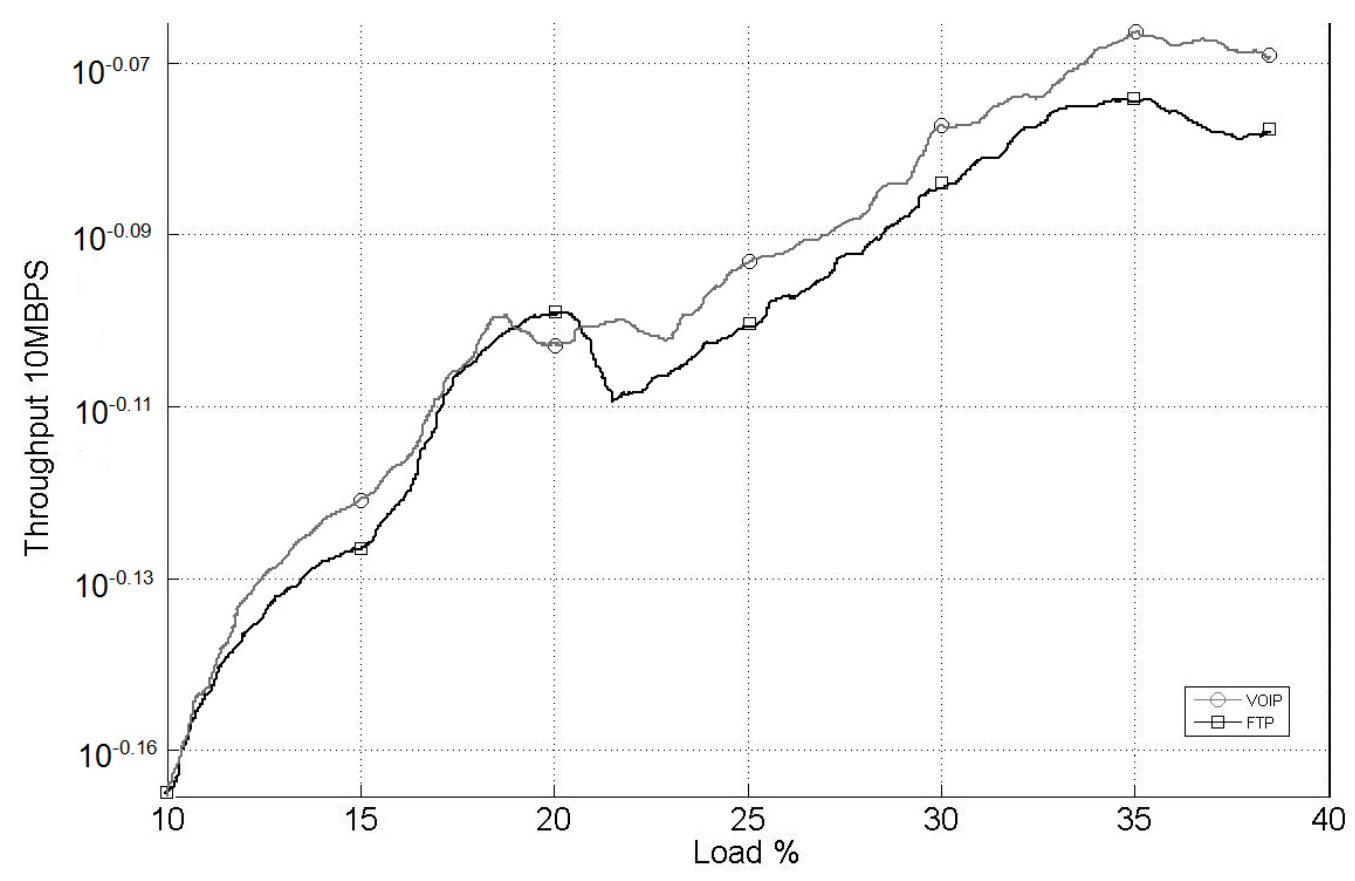

Figure 6-9 Throughput for Test Case 1

In the test case 1, because no differentiated service model is applied, the network uses first-come first-served approach on users. VOIP and FTP are not different in priority. The one first reaches the network is first processed. From Figures 6-8 and 6-9, it can be seen that in terms of delay time and throughput, the VOIP has no advantage over FTP.

In the test case 2 the $\mathrm{CN}$ uses differentiated service model, and VOIP has a very small proportion, and the proportion of FTP is much higher. Because in the differentiated services model, VOIP has higher priority than the FTP, so the network first processes VOIP, and then processes FTP. Therefore, the delay of VOIP has been relatively small. In the throughput, since the proportion of FTP is larger, the output of VOIP reaches saturation point, and becomes difficult to rise. 


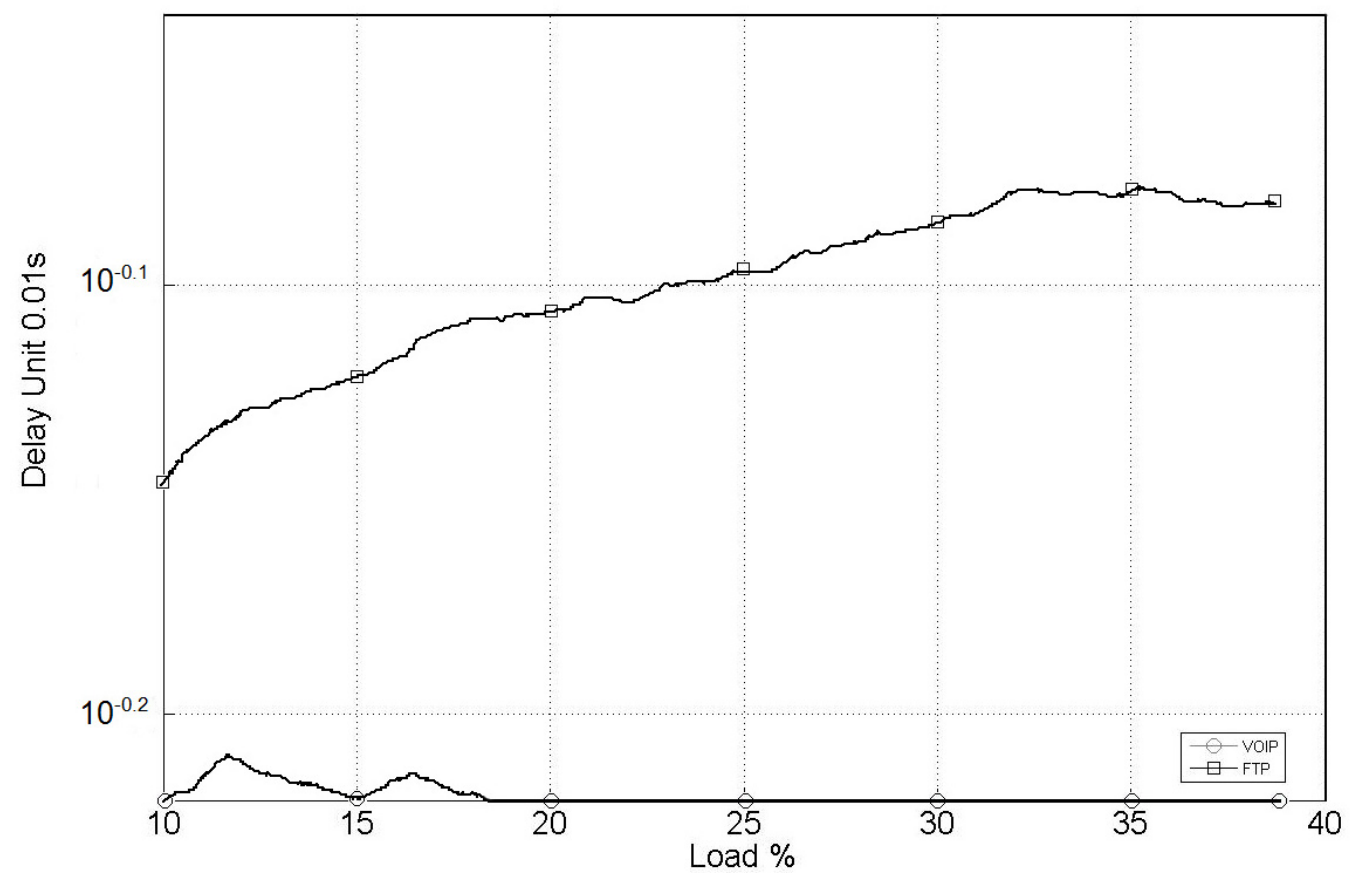

Figure 6-10 Delay for Test Case 2

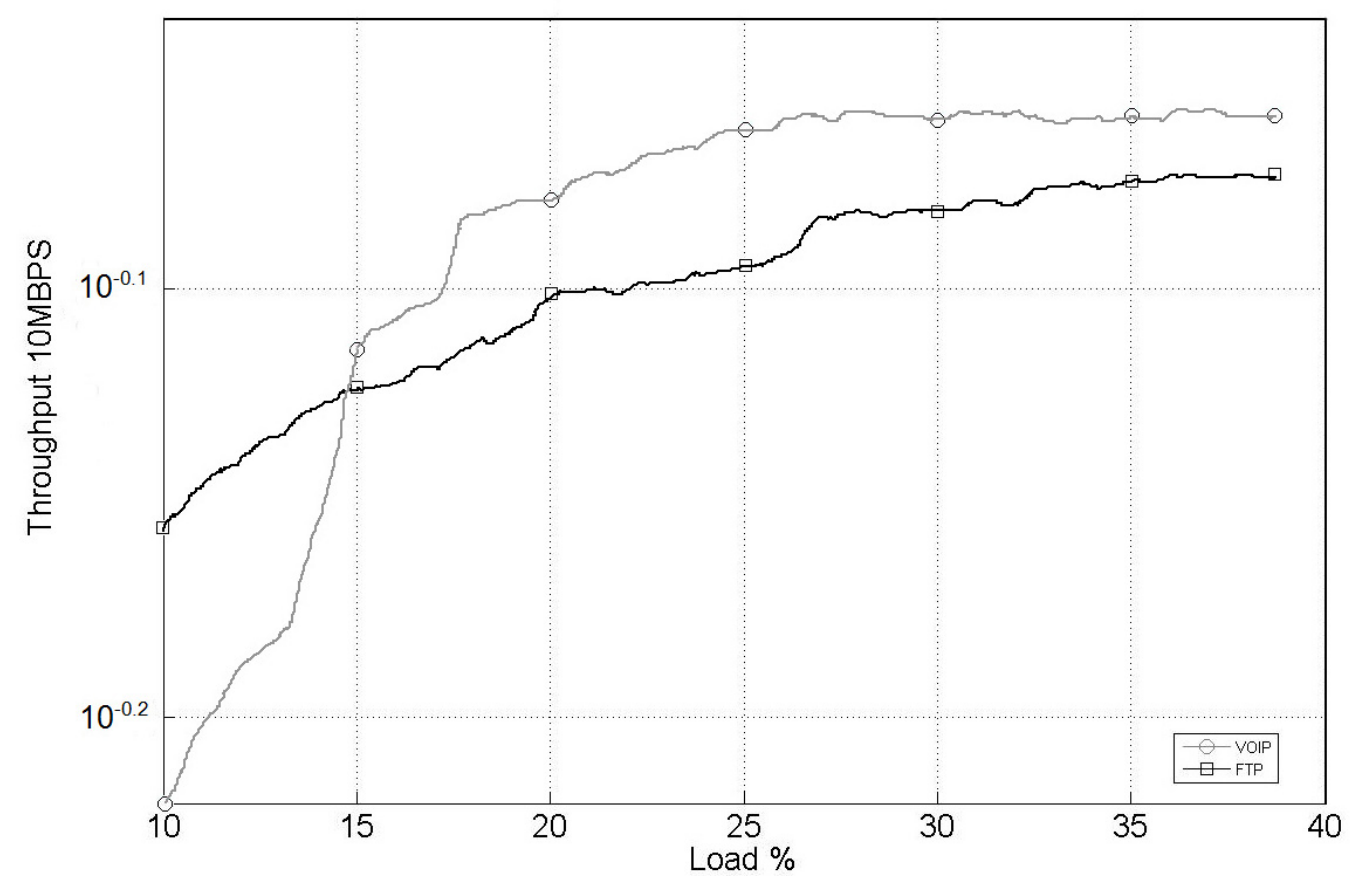

Figure 6-11 Throughput for Test Case 2 


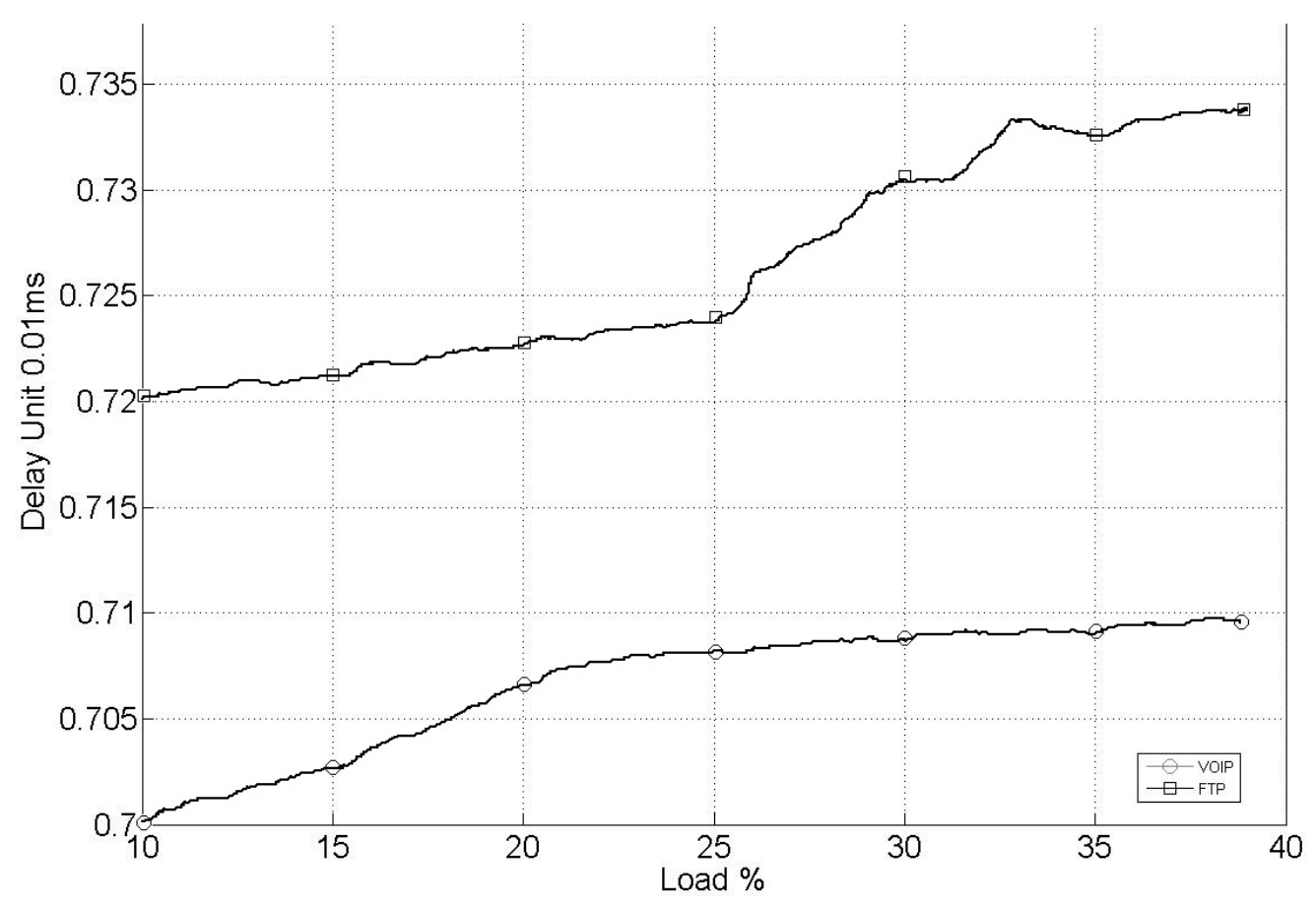

Figure 6-12 Delay for Test Case 3

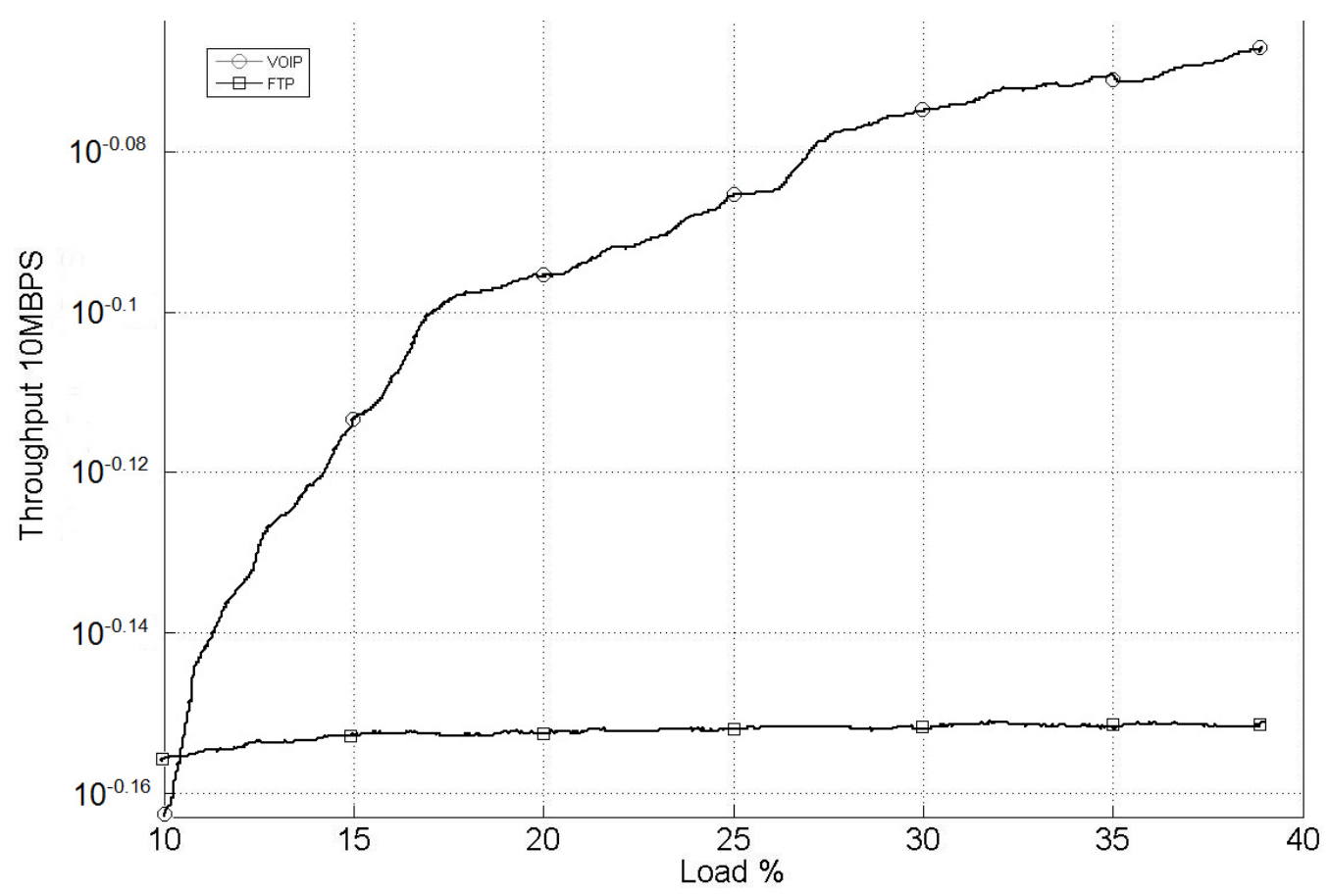

Figure 6-13 Throughput for Test Case 3 
In the test case 3, the proportion of FTP is the same as VOIP. For the delay time, due to scheduling algorithms, the delay of VOIP first increases then decreases. The delay time of FTP keeps rising. For the throughput, FTP maintains a certain flow and does not increase. VOIP throughput rises straightly, and we can not even see the saturation point.

The delay time and throughput diagram of the remaining three kinds of service flows can also be obtained through experiments. The experiment shows that the model in this dissertation appropriately solved the QoS for WCDMA CN, and achieved differentiated services for different service flows. It provides different users with different QoS, so that QoS of users is further assured.

\subsubsection{System Test}

\subsubsection{Test Environment}

To test the proposed system, we used Microsoft Visual C++ 6.0 and Microsoft Media Server to build the simulation model. The simulation network structure is shown in Figure 6-14, and the major equipment and simulation modules are listed in Table 6-1.

Table 6-1 Test Equipments and Simulation Modules

\begin{tabular}{|l|l|l|}
\hline Equipment & Operation System & Modular \\
\hline Center server & Win2000 & Microsoft Media Server, System modular \\
\hline Proxy server & Win2000 & Microsoft Media Server, System modular \\
\hline Linux router & Red Hat Linux & TCP/IP \\
\hline PC & Win2000 & Call generator, Media client \\
\hline
\end{tabular}

Call generator module is responsible for the synchronized arrival of service requests. Media client module is set up as the client software of streaming media system. 


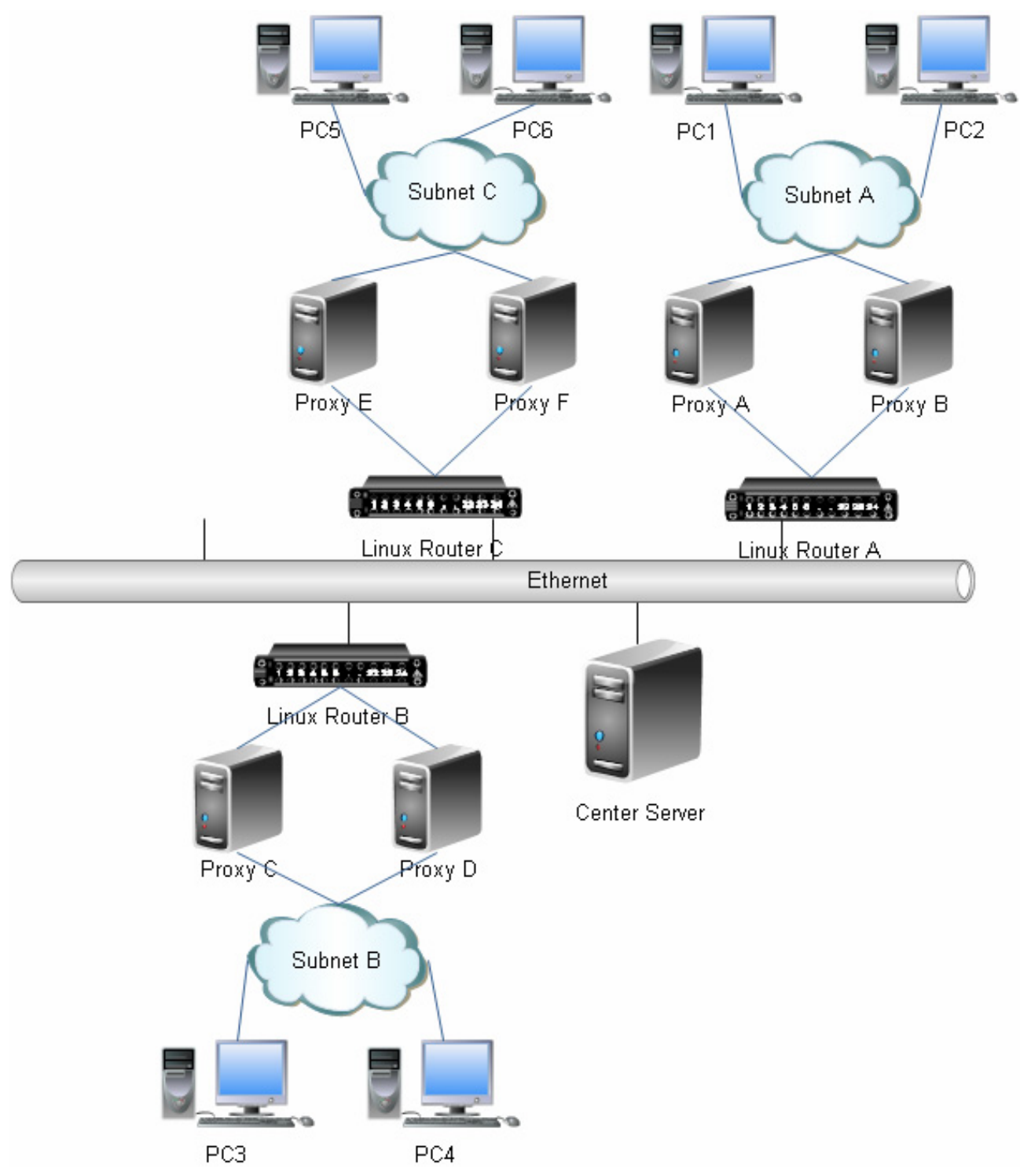

Figure 6-14 Test Network Topology Structure

The following assumptions are made:

- The bandwidth resource that the center server can utilize is $1000 \mathrm{Mb} / \mathrm{s}$.

- The average bandwidth that each proxy server can utilize is $100 \mathrm{Mb} / \mathrm{s}$.

- $\quad$ Each demand for streaming media bandwidth is from $64 \mathrm{~kb} / \mathrm{s}$ to $2000 \mathrm{~kb} / \mathrm{s}$. 


\subsubsection{Test Cases}

The continuing growth of broadband multimedia communication services such as images, broadband video and audio bring about a higher demand for the mobile networks. There are significant differences between multimedia and traditional services. The traditional data services have no strict requirements on QoS assurance. They allow data loss, and can tolerate delay and jitter for a certain period. However, multimedia services cannot tolerate data loss, and have strict requirements on data transmission delay and jitter. In this section, we test the system performance in streaming media environment.

[57] proposed a wireless video-on-demand streaming media system. We use CSBS to represent this type of system. CSBS has the following outstanding features: 1) it can accommodate a large number of clients at the same time; 2) it provides a range of video on demand so that the interaction is improved. In this system, there is no core network operations involved. Therefore, the access delay is smaller. So each media stream only needs a smaller proxy server buffer to complete the functions. In the traditional streaming media system, there is only one-way operation between the server and the client. Because the contents provide by streaming media system are only audio and video data, and there is no two-way interactive data.

In [58-59], the authors designed an interactive streaming system based on MPEG4 coding system, which can be represented as MP4SI. In order to provide efficient MPEG4 streaming, MP4SI adopts a number of suitable network layer and transport layer 
protocols. For IOD, BIFS, OD, and some media data such as static JPEG image that is sensitive to packet loss rate, MP4SI uses the TCP to protect the transmission quality. For those real-time media data, MP4SI uses RTP as the transport layer protocol. In the next generation all IP network, providing high quality streaming services to the client and maximizing system capacity are two contradictions. A good streaming media system must balance between them.

[60] designed a QoS-oriented dynamic streaming media system to transmit streaming data; we use QOAS to express the system. In QOAS, the central server dynamically adjusts the service rate between the server and the clients according to the feedback information of streaming media QoS provided by the clients. [54] also uses simulation results to show that QOAS can efficiently provide QoS.

\subsubsection{Test Results and Analysis}

In the simulation, we calculate the service startup delay according to the following equation:

$$
T_{s}=T_{2}-T_{1}
$$

where $T_{s}$ represents a service start-up delay, $T_{2}$ represents the time when the server start sending media streams to the client, while $T_{1}$ is the time when the client sends the service request. We compare service startup delay of several systems as below. 


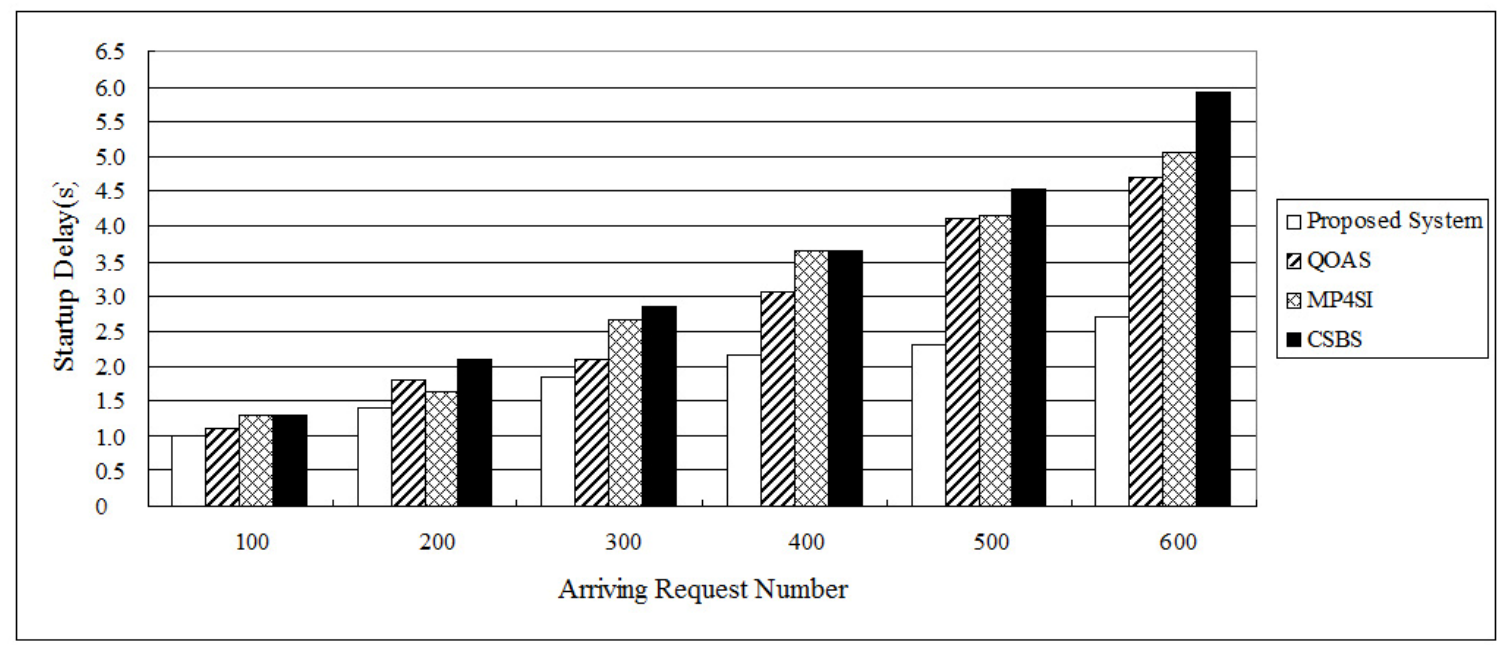

Figure 6-15 Startup Delay Comparison Diagram

In Figure 6-15, with the increasing of simultaneously arrived service requests, startup delay shows a gradually increasing trend. This is because the available system resources gradually decreased, and the service requests progressively reach the system capacity limit.

Table 6-2 Startup Delay Comparison Results

\begin{tabular}{|c|c|c|c|c|}
\hline & \multicolumn{4}{|c|}{ Startup Delay (s) } \\
\hline $\begin{array}{c}\text { Arriving } \\
\text { Request Number }\end{array}$ & $\begin{array}{c}\text { Proposed } \\
\text { System }\end{array}$ & QOAS & MP4SI & CSBS \\
\hline 100 & 1.0 & 1.1 & 2.6 & 1.3 \\
\hline 200 & 1.4 & 1.8 & 3.3 & 2.1 \\
\hline 300 & 1.9 & 2.1 & 5.3 & 2.9 \\
\hline 400 & 2.2 & 3.1 & 7.3 & 3.7 \\
\hline 500 & 2.3 & 4.1 & 8.3 & 4.6 \\
\hline 600 & 2.7 & 4.7 & 10.1 & 5.9 \\
\hline
\end{tabular}

In Table 6-2, we can see that the proposed system has $30 \%$ lower failure rate than the other three systems. This is because in the proposed system, the local proxy server 
serves local service requests, and startup delay is greatly reduced because of the shortened transmission path.

We further compare the trend of average resource function to the service request arrival rate in the four systems. In this simulation experiment, the average resource of a system can be calculated as below.

$$
A_{R}=S_{R} / M
$$

where $A_{R}$ is the average resource. $S_{R}$ represents the total available resource functions of all the proxy servers. $M$ is the total number of proxy servers. Moreover, the service request arrival rate is estimated once per second. We compare system capacity of several systems as below.

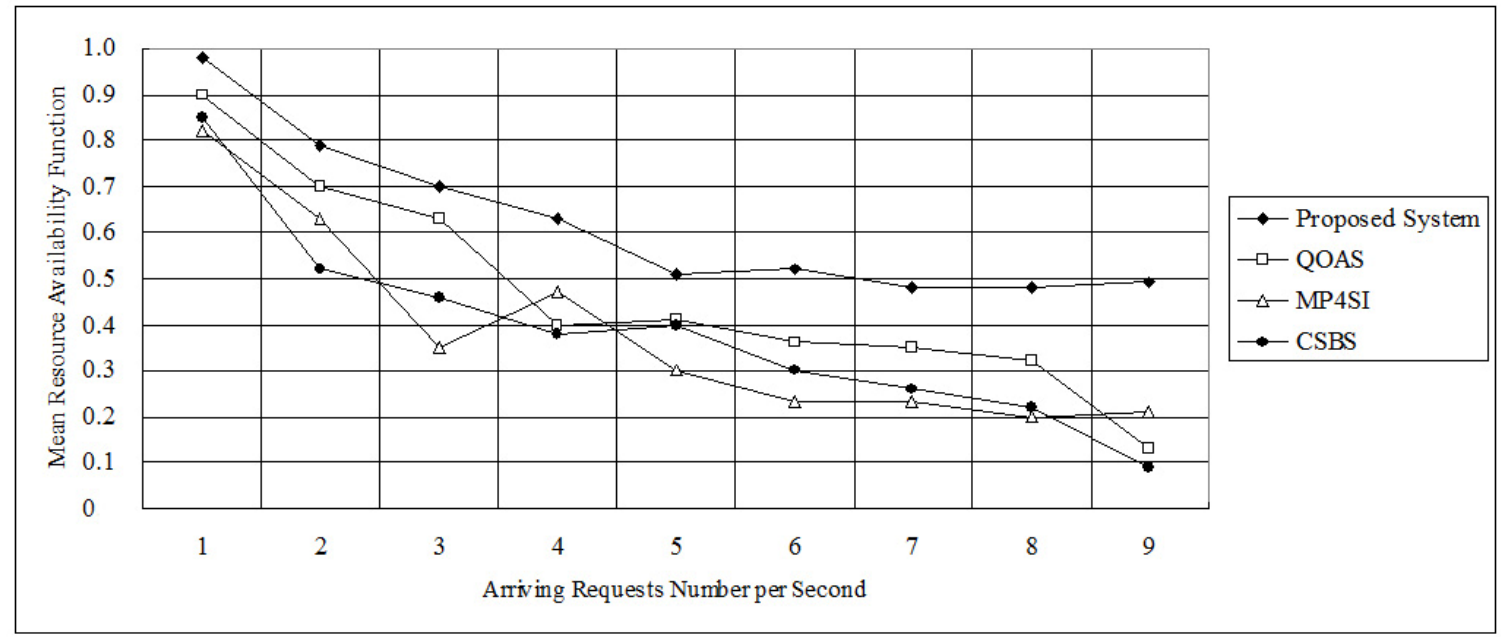

Figure 6-16 System Capacity Comparison Diagram

From Figure 6-16, we can see that when the service request arrival rate is more than 9, all systems show the decreasing trend. 
Table 6-3 System Capacity Comparison Results

\begin{tabular}{|c|c|c|c|c|}
\hline $\begin{array}{c}\text { Arriving Request } \\
\text { Number per second }\end{array}$ & Proposed System & QOAS & MP4SI & CSBS \\
\hline 1 & 0.98 & 0.90 & 0.82 & 0.85 \\
\hline 2 & 0.79 & 0.70 & 0.63 & 0.52 \\
\hline 3 & 0.70 & 0.63 & 0.35 & 0.46 \\
\hline 4 & 0.63 & 0.40 & 0.47 & 0.38 \\
\hline 5 & 0.51 & 0.41 & 0.30 & 0.40 \\
\hline 6 & 0.52 & 0.36 & 0.23 & 0.30 \\
\hline 7 & 0.48 & 0.35 & 0.23 & 0.26 \\
\hline 8 & 0.48 & 0.32 & 0.20 & 0.22 \\
\hline 9 & 0.49 & 0.13 & 0.21 & 0.09 \\
\hline
\end{tabular}

From Table $6-3$, it is seen that the proposed system is $20 \%$ higher than the other three systems. Therefore, the proposed system can simultaneously serve more users, and it achieved DiffServ QoS for WCDMA CN.

\subsection{Summary}

In this chapter, we put forward a complete system including hardware and software design based on IXP2400 to realize QoS DiffServ model for WCDMA CN. According to the proposed programming model in Chapter 3, the system maps the proposed buffer management algorithm PAFD, the packet scheduling algorithm PWFQ and the packet classification algorithm CBNPs to different micro-engines, respectively. Compared to current systems, the simulation results show that startup delay of the proposed system is $30 \%$ lower than that of the other three systems, and system capacity of the proposed system is also $20 \%$ higher than that of the other three systems. Therefore, the proposed system achieves differentiated QoS for WCDMA CN. 


\section{CHAPTER 7}

\section{CONCLUSIONS}

At present, the development of wireless communication networks is at the stage of $3 \mathrm{G}$ evolution. Circuit-switched based wireless communication systems will eventually evolve into end-to-end all IP networks. WCDMA CN and all-IP are the trend of $3 \mathrm{G}$ development. The QoS problem in WCDMA is an extension of the IP QoS problem in mobile communication networks, but QoS in WCDMA networks is more complex than that in general IP networks. DiffServ model does not need complex control signals. It has good scalability and is more suitable for wireless communications, especially for QoS control schemes in large-scale backhaul networks.

An NP is a new System-on-Chip (SoC). It will replace the traditional ASIC chip and become the development direction of future network equipment. The future highspeed network equipment will use NPs as hardware platforms. At present, network research is mainly based on general-purpose processor platforms and simulation environments. A lot of features of NPs are different from ones of general-purpose processors, so lots of algorithms for general-purpose processors cannot be applied to NPs. This dissertation showed some useful attempts on developing algorithms which fully considers the characteristics of NPs. Also, according to different QoS requirements of various services in WCDMA CN, this study mainly studied the implementation schemes of QoS DiffServ model based on NPs. 


\subsection{Major Outcomes}

1) Based on the study on the existing programming models of NPs, the comprehensive programming model that combines HTC and POTs was designed. The solution to solve the packet ordering and resource mutual exclusion problems was also proposed.

2) Buffer management algorithm: According to QoS features in WCDMA, the PAFD algorithm that supports DiffServ was proposed. This algorithm takes into consideration of both fairness and throughput. It has a smooth service curve, and is also self-adaptive to network flows.

3) Packet scheduling algorithm: According to the analysis of QoS characteristics of different service categories in WCDMA, we introduced the PWFQ algorithm which is improved based on WFQ algorithm. In PWFQ algorithm, the fairness of packet scheduling is ensured, and the queue time of packets is reduced. The delay and jitter are also maintained within a small range.

4) Packet classification algorithm: Based on packet compression, rules combination, and tuple lookup algorithms, the CBNPs algorithm was proposed. This algorithm enhances search efficiency and achieves desired time and space complexity.

5) An integrated system supporting DiffServ QoS for WCDMA CN was proposed. The system is implemented with GGSN, SGSN devices based on IXP2400. In the system, differentiated QoS is mapped to different PHBs by using CBNPs algorithm. 
Packet dropping and queue scheduling scheme adopts PAFD and PWFQ algorithm, respectively. Finally, the efficiency and performance of the proposed system was tested in the simulation.

\subsection{Prospective Research Endeavors}

The performance analysis of NPs can be quantitated to optimize the programming model. The network calculus approach which adopts service curve and arrival curve concepts can be adopted for data channel modeling of NPs. Based on this approach, performance of various mapping methods can be analyzed to find the most appropriate resource allocation method. These will be the direction of future research.

The buffer management algorithms studied in this dissertation always concern about the allocation of system buffer resources, while the allocation of bandwidth is given to the packet scheduling algorithms. From the perspective of the entire queue management, buffer management and packet scheduling algorithms are related. They should work together to obtain optimal control effect. Implementation of Joint Buffer Management and Scheduling algorithms (JoBS) on NPs will be a future direction. JoBS provide different levels of services based on bandwidth allocation and adjustment, and optimize the queue operation according to QoS requirements between different service levels. The optimization objective of JoBS is to maintain a static rate allocation and no packet loss. JoBS can provide absolute and relative QoS guarantees, and their performance does not rely on traffic of arrived data flows. In addition, JoBS introduce the rate allocation to the design of buffer management algorithm. This enhances coordination 
with scheduling algorithms and further improves the operation efficiency of the entire system.

Packet classification algorithms are another research area following routing searching algorithms. The study on packet classification references point positioning and other related knowledge in the computational geometry. Recently a lot of packet classification algorithms were proposed, and some of them are quite outstanding. However, they are all based on the TRY tree structure. Because of the special code space of NPs like IXP2400, the tree structure access is very difficult. The tree structure must be converted into a linear structure to facilitate micro-engines searching. Converting various TRY tree structure into reasonable linear structures and finding the fastest access method will be the future research direction of packet classification algorithms.

With the development of $3 \mathrm{G}$ network, the $\mathrm{CN}$ will develop into all-IP network. Multiple protocol Label Switching (MPLS) technology has significant expansion in flow control and QoS support for IP networks. MPLS divides network elements into the core and edges, and completes complex processing tasks on edges of the network, which is called edge intelligence. MPLS exactly matches the forwarding packets based on fixedlength short-labels. This simplifies the forwarding scheme and is conducive to fastforward packet in CN. The research on implementation of MPLS QoS on NP will be the future direction. 


\section{LIST OF REFERENCES}

1 A. Jamalipoura, P. Lorenz, "End-to-end QoS support for IP and multimedia traffic in heterogeneous mobile networks", Computer Communications, Vol.29, pp.671$682,2006$.

2 3GPP TSG, TS 23. 922. Architecture for an All IP Network, March 2003.

3 3GPP TSG, TS 24. 008. Mobile Radio Interface Layer 3 Specification, Core Network Protocols, March 2003.

4 K. Peng, Y. Lu, "The Research of the Implement of UMTS QoS DiffServ on IXP2400", Proc. of IEEE International Conference on Wireless Communication, Networking and Mobile Computing, pp. 840-843, September 23-26, 2005.

$5 \quad$ K. Peng, Y. Lu, "Research of UMTS Core Network Firewall Based on IXP1200", Proc. of the $3^{\text {rd }}$ International Conference on Mechatronics and Information Technology, Vol. v6041September 20-23, 2005.

6 F. Siddiqui, S. Zeadally, "Mobility Management across Hybrid Wireless Networks-trends and Challenges", Computer Communications, Vol.29, pp.1363$1385,2006$.

7 V. G. Ozianyi, N. Ventura, "A Novel Pricing Approach for QoS Enabled 3G Networks", Proceedings of the IEEE LCN Conference, pp.578-586, 2005.

8 R. Koodli, M. Puuskar, "Supporting Packet-data QoS in Next-Generation Cellular Networks”, IEEE Communication Magazine, Vol.39, No.2, pp.180-188, 2001.

9 V. G. Ozianyi, N. Ventura and E. Golovins, "A Novel Pricing Approach to Support QoS in 3G networks”, Computer Networks, Vol.52, pp.1433-1450, 2008.

10 H. Chaskar, R. Koodli, "MPTS and DiffServ for UMTS QoS in GPRS Core Network Architecture", Proceedings of NET, pp.244-249, Stockholm, 2001.

11 S. Maniatis, "QoS Issues in the Converged 3G Wireless and Wired Network", IEEE Communications Magazine, Vol.40, No.8, pp.44-53, 2002.

12 V. Marques, R. Aguiar and P. Pacyna, “An Architecture Supporting End-to-end QoS with User Mobility for Systems beyond 3rd Generation”, 1st Mobile \& Wireless Telecommunications Summit, pp.858-862, Thessaloniki, 2002.

13 K. Peng, Y. Lu, “A Novel QoS DiffServ Model of WCDMA Research and Implement on Network Processor", Proc. of 2005 Global Mobile Congress, pp.265-270, October 10-12, 2005. 
14 T. Hoßfeld, A. Binzenhofer, "Analysis of Skype VoIP Traffic in UMTS: End-toend QoS and QoE Measurements", Computer Networks, Vol.52, pp.650-666, 2008.

15 F. Zaraia, N. Boudrigaa and M. S. Obaidatb, "Performance Evaluation of A Novel Scheme for QoS Provision in UTRA TDD”, Computer Communications, Vol.29, pp.969-982, 2006.

16 F. Palmieri, "A MPLS-based Architecture for Scalable QoS and Traffic Engineering in Converged Multi-service Mobile IP Networks", Computer Networks, Vol.47, pp.257-269, 2005.

17 J. Diederich, L. Wolf and M. Zitterbart, "A Mobile Differentiated Services QoS Model”, Computer Communications, 2004.

18 S. Andersen, A. Duric, H. Astrom, R. Hagen, W. Kleijn and J. Linden, "RFC 3951: internet Low Bit Rate Codec (iLBC)”, 2004.

19 H. Shimonishi, T. Murase, "A Network Processor Architecture for Flexible QoS Control in Very High-speed Line Interfaces", Proceedings of the 2001 IEEE Workshop on High Performance Switching \& Routing, pp.402-406, Dallas, 2001.

20 Mäder A., Wagner B., Hoßfeld T., Staehle D. and Barth H., "Measurements in A Laboratory UMTS Network with Time-varying Loads and Different Admission Control Strategies", Proceedings of IPS-MoMe, Salzburg, Austria, 2006.

21 Intel Corporation, Development Tools User's Guide, 2003.

22 Intel Corporation, IXA Portability Framework Developer’s Manual, 2003.

23 Intel Corporation, Intel IXP2400 Network Processor Hardware Reference Manual, 2003.

24 Intel Corporation, Intel IXP2400 Network Processor-2nd Generation Intel NPU, 2002.

25 Global IP Sound, iSAC, http://www.globalipsound.com/datasheets/iSAC.pdf, 2006.

26 J. Erik and R. Aaron, IXP2400/2800 Programming, Intel Press, 2004.

27 M. Labrecque, Towards a Compilation Infrastructure for Network Processors, Master's Thesis, University of Toronto, pp.7-11, 2006.

28 L. Thiele, S. Chakraborty, M. Gries, A. Maxiaguine and J. Greutert, "Embedded Software in Network Processors Models and Algorithms", Proceedings of the 1st 
Workshop on Embedded Software, Lecture Notes in Computer Science, Lake Tahoe, CA, 2001.

29 F. Buccafurri et. al., "Analysis of QoS in Cooperative Services for Real-time Applications", Data \& Knowledge Engineering, Vol.67, No.3, 2008.

30 Y. Ito, S. Tasaka, "Feasibility of QoS control based on QoS Mapping over IP Networks", Computer Communications, Vol.31, No.10, 2008.

31 A. Tiwaria and A. Sahoo, "Providing QoS in OSPF based Best Effort Network Using Load Sensitive Routing", Simulation Modelling Practice and Theory, Vol.15, No.4, 2007.

32 D. A. Menascéa, H. Ruana, and H. Gomaa, "QoS Management in Serviceoriented Architectures", Performance Evaluation, Vol.64, No.7, 2007.

S. Floyd, V. Jacobson, "Random Early Detection Gateways for Congestion Avoidance", IEEE/ACM Transactions on Networking (TON), August, 1993.

34 N. Masayoshi, "Improving the Performance of Active Buffer Management with Per-flow Information”, IEEE Communications Letters, Vol.6, No.7, July, 2002.

35 RFC: Recommendations on Queue Management and Congestion Avoidance in the Internet.

36 W. Feng, K. G. Shin, D. D. Kandlur, and D. Saha, "The Blue Active Queue Management Algorithms", IEEE/ACM Transactions on Networking Vol.10, No.4, pp.513-528, 2002.

37 C.V. Hollot, V. Misra, D. Towsley, and W. Gong, "Analysis and Design of Controllers for AQM Routers Supporting TCP Flows", IEEE Transactions on Automatic Control, Vol.47, No.6, pp.945-959, 2002.

38 M. Ghaderi and R. Boutaba, "Call Admission Control for Voice/data Integration in Broadband Wireless Networks", IEEE Transactions on Mobile Computing, Vol.5, No.3, 2006.

39 G. Ascia, V. Catania, D. Panno, "An Efficient Buffer Management Policy Based On an Integrated Fuzzy-Ga Approach", Proc. of IEEE INFOCOM 2002, New York, USA, Jun. 23-27, 2002.

40 E. L. Hahne, A. K. Choudhury, "Dynamic Queue Length Thresholds for Multiple Loss Priorities", IEEE/ACM Transactions on Networking, Vol.10, No.3, June 2002.

41 A. Demers, S. Keshav, and S. Shenker, "Analysis and Simulation of a Fair Queuing Algorithm", Proceedings of ACM SIGCOMM, pp.1-12, Sep. 1989. 
42 L. Zhang, "VirtualClock: A New Traffic Control Algorithm for Packet Switching Network”, Proceeding of ACM SIGCOMM'90, pp.19-29, August, 1990.

43 V. Srinivasan, G. Varghese, "Fast IP Lookups Using Controlled Prefix Expansion”, ACM, Trans. on Computer Systems, Vol.17, No.1, pp.1-40, 1999.

44 Wikipedia, http://en.wikipedia.org/wiki/Weighted_round_robin.

45 M. Shreedhar, G. Varghese, "Efficient Fair Queuing Using Deficit Round Robin", ACM SIGCOMM Computer Communication Review, Vol.25, No.4, pp.231, October, 1995.

46 A. K. Parekh, G. R. Gallager, "A Generalized Processor Sharing Approach to Flow Control in Integrated Services Networks: The Multiple Node Access", IEEE/ACM Trans. on Networking, Vol.2, No.2, pp.137-150, 1994.

47 V. G. Ozianyi, A Novel Pricing Approach to Support QoS in 3G Networks, Master's Thesis, University of Cape Town, Private Bag, 7701 Rondebosch, South Africa, 2006.

48 K. Peng, Y. Lu, "A Novel Packet Classification Algorithm for Information Security Firewall", Proc. of International Conference on Space Information Technology, Vol. v5985 part1, November 19-20, 2005.

49 Texas Instruments, OMAP3430, http://focus.ti.com/, 2006.

50 W. Fang, N. Seddigh, and B. Nandy, A Time Sliding Window Three Color Marker (tswTCM), RFC2859, June, 2000.

51 J. V. Lunteren, A. P. J. Engbersen, "Multi-field Packet Classification Using Ternary CAM”, Electronics Letters, Vol.38, No.1, pp.21-23, 2002.

52 V. Srinivasan, G. Varghese, and S. Suri, "Fast and Scalable Layer Four Switching", ACM SIGCOMM Computer Communication Review, Vol.28, No.4, pp.191-202, 1998.

53 M. Buddhikot, S. Suri, and M. Waldvogel, "Space Decomposition Techniques for Fast Layer-4 Switching", Proc. of Conf. on Protocols for High Speed Networks, Salem, MA, USA, pp.25-41, 1999.

54 P. Gupta, N. McKeown, "Packet Classification Using Hierarchical Intelligent Cuttings", IEEE Micro, Vol.20, No.1, pp.34-41, 2000.

55 A. Brodnik, S. Carlsson, M. Degermark, et. al., "Small Forwarding Tables for Fast Routing Lookup”, Proc. of ACM SIGCOMM, Cannes, France, 1997. 
56 J. Xu, M. Singhal, and J. Degroat, "A Novel Cache Architecture to Support Layer-four Packet Classification at Memory Access Speeds", Proc. of INFOCOM, Tel Aviv, Israel, 2000.

57 Y. W. Leung, T. K. C. Chan, "Design of an Interactive Video-on-demand System", IEEE Transactions on Multimedia, Vol.5, No. 1, pp.130-140, Mar. 2003.

58 H. C. Kim, K. Kim and S. Min, "Design and Implementation of Streaming System of MPEG- 4 based Interactive Contents over IP Networks", Proc. of Digital and Computational Video 2002, pp.100-107, Nov. 14-15, 2002.

59 H. C. Kim, J. M. Ieong and K. Kim, "Development of Interactive Contents Streaming System based on MPEG-4", Proc. of the 6th International Conference on Advanced Communication Technology, Vol.2, pp.751-755, Feb.2004.

60 G. M. Muntean, P. Perry and L. Murphy, "A New Adaptive Multimedia Streaming System of All-IP Multi-service Networks", IEEE Transactions on Broadcasting, Vol. 50, No. 1, pp. 1-10, March 2004. 
VITA

\section{YECHANG FANG}

June 10, 1981

$2000-2004$

$2005-2006$

$2006-2007$

$2007-2010$
Born, Puyang, Henan, China

B.S., Telecommunication Engineering

Beihang University

Beijing, China

Program Coordinator

Software College, Beihang University

Beijing, China

M.S., Electrical Engineering

Florida International University

Miami, FL

Client Representative

IBM China

Beijing, China

Doctoral Candidate in Electrical Engineering

Florida International University

Miami, FL

\section{PUBLICATIONS AND PRESENTATIONS}

1. Y. Fang, K. Yen, A. Caballero, Wu, N. "A Multidimensional Packet Classification Algorithm Based on Network Processors", Proceedings of the 12th WSEAS International Conference on Automatic Control, Modeling and Simulation (ACMOS’10), pp. 231-234, Catania, Italy, May 29-31, 2010.

2. A. Caballero, K. Yen, Y. Fang, J. L. Abreu, "Method for Classification in Interval-Valued Information Systems (Plenary Lecture)", Proceedings of the 12th WSEAS International Conference on Automatic control, Modeling and Simulation (ACMOS'10), pp. 242-247, Catania, Italy, May 29-31,2010.

3. Y. Du, Y. Fang, N. Wu and K. K. Yen, "Performance Analysis for Complex FastICA Algorithms in MIMO OFDM Systems", Proc. of the 2nd International Conference on Future Computer and Communication, Wuhan, May 21-24, 2010. 
4. Y. Fang, K. Yen, D. Pan, and Z. Shun, "Buffer Management Algorithm Design and Implementation Based on Network Processors", International Journal of Computer Science and Information Security, vol. 8, no. 1, pp 1-8, April 2010.

5. N. WU, Y. FANG, Y. DU, and K. K. YEN, "Mutiuser Scheduling Schemes for MIMO-OFDM Systems", Proc. of the 74th Florida Academy of Sciences Annual Meeting, Fort Pierce, Florida, USA, March 19-20, 2010.

6. Y. DU, N. WU, Y. FANG, and K. K. YEN, "A Comparison of Different Contrast Functions of FastICA Algorithms in a MIMO-OFDM System", Proc. of the 74th Florida Academy of Sciences Annual Meeting, Fort Pierce, Florida, USA, March 19-20, 2010.

7. Y. Fang, K. Yen, A. Caballero, N. Wu, "Design and Realization of Transportation Information Acquisition System Based on 3G and DSP", Journal of E-business Technology and Strategy, vol. 6, no. 1, pp 61-69, January-March 2010.

8. A. Caballero, K. Yen, Y. Fang, "Analysis of Classification in Interval-Valued Information Systems: A Case Study", Proc. of the 5th Int'l Conf. on Information, Kyoto, Japan, November 6-9, 2009.

9. Y. Fang, K. Yen, A. Caballero, Nansong Wu, "Design of a New Transportation Information Acquisition System Based on 3rd Generation Mobile Communication Technology", Proc. of the 5th Int'l Conf. on Information, Kyoto, Japan, November 6-9, 2009.

10. Y. Fang, N. Wu, Y. Du, K. Yen, "A Queue Management Algorithm Based on Network Processors", Proc. of the 8th Int'l Conf. on Information and Management Sciences, pp. 289-293, Kunming, China, July 20-28, 2009.

11. M. Zhang, L. Sun, Y. Fang, S. Yang, "iGridMedia: The system to provide low delay peer-to-peer live streaming service over internet", Peer-to-Peer Networking and Applications, Springer, June 2009.

12. K. Yen, A. Caballero, Y. Fang, "Iris Classification Using Rough Sets and Fuzzy Pattern Classification Techniques", Proc. of the 7th Int'1 Conf. on Information and Management Sciences, pp. 464-468, Urumchi, China, August 12-19, 2008.

13. A. Caballero, K. Yen, Y. Fang, "Classification with Diffuse or Incomplete Information", WSEAS Trans. on Systems and Control, vol. 3, no. 6, pp 617-626, June 2008. 